SEASONAL VARIABILITY IN THE INTERMEDIATE WATERS

OF THE EASTERN NORTH ATIANTIC

by

NANCY AMANDA BRAY

B.S., University of California, Berkeley

(1975)

\begin{tabular}{c} 
MARINE \\
BIOLOGICAL \\
LABORATORY \\
\hline LIBR RARY \\
\hline $\begin{array}{c}\text { WOODS HOLE, MASS. } \\
\text { W. H. O. I. }\end{array}$ \\
\hline
\end{tabular}

SUBMITTED IN PARTIAL FULFILIMENT

OF THE REQUIREMENTS FOR THE

DEGREE OF

DOCTOR OF PHILOSOPHY

at the

MASSACHUSETTS INSTITUTE OF TECHNOLOGY

and the

WOODS HOLE OCEANOGRAPHIC INSTITUTION

August 1980

Signature of Author

Joint Program in Oceanography, Massachuséts Institute of Technology

- Woods Hole Oceanographic Institution, and Department of Earth and

Planetary Sciences, and Department of Meteorology, Massachusetts

Institute of Technology, August 1980.

Certified by

Thesis Supervisor

Accepted by

Chairman, Joint Oceanography Commitee in Earth Sciences,

Massachusetts Institute of Technology - Woods Hole Oceanographic Institution 


\title{
SEASONAL VARIABILITY IN THE INTERMEDIATE WATERS \\ OF THE EASTERN NORTH ATLANTIC
}

\author{
by \\ NANCY AMANDA BRAY
}

\begin{abstract}
Submitted to the Joint Oceanographic Committee in the Earth Sciences, Massachusetts Institute of Technology and Woods Hole Oceanographic Institution, on August 15, 1980, in Partial Fulfillment of the Requirements for the Degree of Doctor of Philosophy
\end{abstract}

ABSTRACT

Observational evidence of seasonal variability below the main thermocline in the eastern North Atlantic is described, and a theoretical model of oceanic response to seasonally varying windstress forcing is constructed to assist in the interpretation of the observations. The observations are historical conductivity-temperature-depth data from the Bay of Biscay region $\left(2^{\circ}\right.$ to $20^{\circ} \mathrm{W}, 42^{\circ}$ to $\left.52^{\circ} \mathrm{N}\right)$, a series of eleven cruises over the three years 1972 through 1974, spaced approximately three months apart. The analysis of the observations utilizes a new technique for identifying the adiabatically leveled density field corresponding to the observed density field. The distribution of salinity anomaly along the leveled surfaces is examined, as are the vertical displacements of observed density surfaces from the leveled reference surfaces, and the available potential energy. Seasonal variations in salinity anomaly and vertical displacement occur as westward propagating disturbances with zonal wavelength $390( \pm 50) \mathrm{km}$, phase $71( \pm 30)$ days from 1 January, and maximum amplitudes of \pm 30 ppm and $\pm 20 \mathrm{ab}$ respectively. The leveled density field varies seasonally with an amplitude corresponding to a thermocline displacement of $\pm 15 \mathrm{db}$.

The observations are consistent with the predictions of a model in which an ocean of variable stratification with a surface mixed layer and an eastern boundary is forced by seasonal changes in a sinusoidal windstress pattern, when windstress parameters calculated from the observations of Bunker and Worthington (1976) are applied.

Thesis Supervisor: Nicholas P. Fofonoff

Title: Senior Scientist

Department of Physical Oceanography

Woods Hole Oceanographic Institution 
TABLE OF CONTENTS

Page

ABSTRACT. . . . . . : . . . . . . . . . . . . . 2

IIST OF FIGURES. . . . . . . . . . . . . . . . . 4

LIST OF TABLES. . . . . . . . . . . . . . . . . . 6

Chapter I: INTRODUCTION. . . . . . . . . . . . . . 7

1.1 Introduction. . . . . . . . . . . . . . 7

1.2 Earlier Work. . . . ............... 11

1.3 Potential Energy. . . . . . . . ........ 18

1.4 Procedure for Calculating the Adiabatically Leveled

Steric Field................. . 33

Chapter 2: OBSERVATIONS:................... 38

2.1 Introduction. . . . . . . . . . . . . . . 38

2.2 Application of the Adiabatic Leveling Technique . . . . 43

2.3 Mean Fields and Non-seasonal Variability. . . . . . 51

2.4 Seasonal Variability. . . . . . . . . . . . 83

2.5 Discussion. . . . . . . . . . . . . 94

Chapter 3: MODEL . . . . . . . . . . . . . . . 96

3.1 Introduction. . . . . . . . . . . . . . . 96

3.2 Model . . . . . . . . . . . . . . . . 98

3.3 Model Results ..................... 108

3.4 Comparison of Model and Observations. . . . . . . . 119

3.5 Discussion.................... 136

Chapter 4 : CONCLUSIONS ................. 142

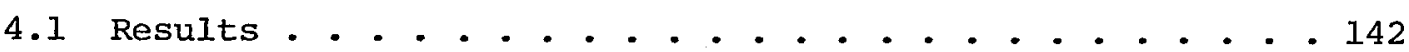

4.2 Implications. . . . . . . . . . . ..... 148

4.3 Further Work. . . . . . . . ........ 153

ACKNOWLEDGEMENTS. . . . . . . . . . . . . . . 155

REFERENCES. . . . . . . . . . . . . . . . . . 156

APPENDIX A. . . . . . . . . . . . . . . . . . . . 159

APPENDIX B. . . . . . . . . . . . . . . . . . . 163

BIOGRAPHICAL SKETCH. . . . . . . . . . . . . . . . 165 
LIST OF FIGURES

Chapter 1:

$\underline{\text { Page }}$

1.1 Schematic of displacements in an initially level steric field.............. . . 22

1.2 Schematic of the adiabatic leveling calculation. . . 30 Chapter 2:

2.1 Map of Phygas station locations. . . . . . . . . 42

2.2 Reference steric anomaly for Phygas $42 . . . . . . .53$

2.3 Average buoyancy frequency N . . . . . . . 55

2.4 Average $\theta$ vs. S. . . . . . . . . . . 57

2.5 Rms vertical displacements ............ 60

2.6 APE per unit mass for Phygas 42; includes estimates of eddy $\mathrm{KE}$ from current meter records ....... 62

2.7 Comparison of APE per unit mass and $.5 \overline{\mathrm{N}^{2}} \overline{\zeta^{2}}$ for Phygas 42 . . . . . . . . . . . . . . 64

2.8 Dynamic height 500/1000 db. Phygas 22, 23, 24... 68

2.9 Dynamic height 500/1000 db. Phygas 31, 32, 33, 34.. 69

2.10 Dynamic height 500/1000 db. Phygas 41, 42, 43, 44.. 70

2.11 Dynamic height as a function of time and zonal position. . . . . . . . . . . . 72

2.12 Average salinity anomaly as a function of depth. . . 74

2.13 Salinity anomaly on reference steric surface corresponding to $1000 \mathrm{db}$. Phygas 22, 23, 24 ... . 77

2.14 Same as 2.13, but for Phygas $31,32,33,34 . \ldots 78$

2.15 Same as 2.13, but for Phygas 41, 42, 43, 44. .... 79

2.16 Statistical significance of regression coefficients for a range of frequencies. . . . . . . . 85

2.17 Salinity anomaly at origin as a function of depth and time.................. 88 


\section{LIST OF FIGURES (Contd)}

Chapter 2 (Contd):

Page

2.18 Reference steric anomaly as a function of depth and time................. 90

2.19 GPE for all cruises. . . . . . . . . . . 93

Chapter 3:

3.1 Stream, function $\hat{\mathrm{p}}(\mathrm{z})$.............. 113

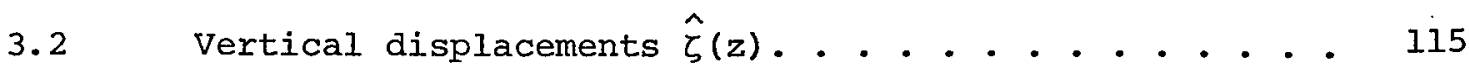

3.3 Meridional velocities $\hat{v}(z)$. . . . . . . . . 117

3.4 Windstress zonal and meridional sections....... 121

3.5 Seasonal averages of windstress along $45^{\circ} \mathrm{N}$ between $0^{\circ}$ and $70^{\circ} \mathrm{W}$. . . . . . . . . . . 123

3.6 Salinity anomaly amplitudes: comparison of model and observations. . . . . . . . . . $\quad .127$

3.7 Statistical significance of regression coefficient for a range of parameters . . . . . . . . 130

3.8 Displacement amplitudes: comparison of model and observations. ............... 132

3.9 APE per unit mass: comparison of model and observations.................. 135

Chapter 4:

4.1 Lateral tracer gradients required to detect a range of particle velocities. . . . . . . . . . 
LIST OF TABLES

\section{Chapter 2}

2.I Description of Phygas Cruises. .......... 40

2.2 Calibration Errors ................ 44

2.3 Regression Parameters for Adiabatic Leveling . . . . . 45

2.4 Typical Error Values for Derived Variables . . . . . 50

2.5 Potential Energy per Unit Area . . . . . . . . 65

2.6 Gradients of Salinity Anomaly at Origin. . . . . . . 80

2.7 Zonal Gradients of Temperature Finestructure . . . . . 82

\section{Chapter 3}

3.1 Model Parameters ................ 109

Eigenvalues and Corresponding Wavelengths and
Phase speeds. . . . . . . . . . . 110

3.3 Effects of Friction and Diffusion. ........... 141 


\section{Chapter 1}

\subsection{Introduction}

Seasonal variations in the strength of the wind are comparable in magnitude to the mean wind over much of the world's ocean. Consequently, significant seasonal fluctuations in the wind driven ocean circulation might be anticipated. The ocean adjusts to periodic forcing at the surface through a combination of barotropic and baroclinic motion. The barotropic motion, which is independent of the stratification, represents the response of the water column as a whole to the changes in the surface. mass field which occur as a result of the stress exerted on the surface by the wind. Baroclinic motion exists only if the fluid is stratified, and represents the adjustment of the density field to the imposed forcing. The time scale for baroclinic adjustment is longer than for barotropic adjustment. Veronis and Stommel (1956) demonstrated that the seasonal time scale represents a crossover point between high frequency forcing with dominantly barotropic response and very low frequency forcing with dominantly baroclinic response. For seasonal forcing, the barotropic and baroclinic components are of approximately equal importance, so that the adjustment of the ocean to seasonally varying windstress is not confined to the surface layers. In addition to seasonal variations in the wind, there is a seasonal cycle to the heating and cooling of the ocean surface, which may cause significant seasonal variations in the large scale thermohaline circulation of the ocean.

The general context of this work is the presentation and interpretation of observational and theoretical evidence for seasonal variability in the ocean at depths below the region of the direct atmos:-.. pheric influence. The observational evidence is -taken from a three year 
series of hydrographic cruises in the eastern North Atlantic. A theoretical model of the oceanic response to seasonal windstress forcing is constructed using the observed oceanic parameters, and the model prediction is compared with the observed variability.

Veronis and Stommel (1956) demonstrated that seasonal forcing by a moving wind stress pattern in an unbounded ocean results in very small vertical and horizontal displacements in the main thermocline. In that case the length scale of the forcing and response are the same. However, the presence of lateral boundaries imposes an additional constraint on the system of no flow into the boundary. In general, the forced response of the unbounded ocean cannot satisfy that boundary condition, so that free motions (solutions to the unforced equations) must be added to the forced solution. If the system of equations is linear, the superposition of free and forced motions which satisfies the lateral boundary condition is also a solution to the governing equations. The scales of the free motions are determined by the geometry, the frequency, the stratification, and the lateral boundary condition. In certain cases, the dominant free response, which necessarily has a wave amplitude comparable to that of the forced response, may have a horizontal scale much smaller than that of the forcing. In that circumstance, the vertical and horizontal particle displacements of the dominant free response will be larger than those of the forced response. This is due to the geometrical fact. that the same amount of energy (which is proportional to the square of the wave amplitude) distributed over a smaller area will result in larger displacements. If the free response scale is small enough, the response of the ocean to large scale atmospheric forcing can be detected observationally, even though the forced response alone is too weak to be observed: 
The primary hypothesis of this work is that the observed annual variations in wind stress curl over the North Atlantic, in combination with a meridional barrier at the eastern edge of the ocean, are capable of producing seasonal variability below the main thermocline of sufficient amplitude to be detected observationally in the eastern part of the basin. There is a corollary hypothesis that low frequency signals which involve small particle velocities can be observed indirectly if the motion occurs in a region of strong horizontal gradient of any tracer, by following the movement of parcels tagged by an initial tracer condition. The strong ambient gradient acts to amplify the signal, in the sense that lateral displacements will result in anomalies from the initial state of the tracer involved. Therefore, information from tracer distributions along density surfaces can be used to detect velocity signals too small to be resolved by standard Eulerian current measurements, provided the lateral tracer gradients are sufficiently strong.

Conceptually, this work is concerned with the transfer of energy from large scales in the atmosphere to considerably smaller scales in the ocean. Although the details of the transfer process itself are not studied, the estimation of the observed available potential energy in the ocean is discussed at length. The available potential energy or APE is that part of the total potential energy which is actually available for conversion to kinetic energy. Formally, it is defined as the difference between the horizontally averaged total potential energy and the adiabatic minimum in potential energy obtained when surfaces of constant potential density coincide with geopotential surfaces (i.e. potential energy corresponding to the adiabatically leveled reference density field). Previous estimates of APE in which effects of compressibility are ignored 
and reference states other than the adiabatically leveled field are used do not allow the precise evaluation of errors in the estimate: .

A computational technique for determining the adiabatically leveled reference density field using the full equation of state is described below whose accuracy is limited only by the sampling and measurement errors of the observations. The technique permits the evaluation of the effects of measurement and finestructure errors on the calculation of APE, as well as the errors which result from neglecting compressibility in the Boussinesq estimate of APE. Because geopotential is not measured accurately in the ocean, pressure surfaces rather than geopotential surfaces are used in the definition of the reference state. The leveled density surfaces are also the correct surfaces for examining tracer distributions, and are employed in all calculations involving tracers in this work. The adiabatic leveling technique provides a precise and consistent overall framework for the analysis of hydrographic data in terms of both energetics and tracer distributions. 


\subsection{Earlier Work}

There is little observational evidence for seasonal variability in the oceans below the surface layers. The reason for this is two-fold: only recently have observations of sufficiently long duration been available, and, except at low latitudes, the expected annual signal is small compared to the energetic western boundary currents and mesoscale eddies. In the near equatorial region where the baroclinic response time of the ocean is shorter than at higher latitudes, seasonal variability has been observed. White (1977) found evidence for propagation of long baroclinic Rossby waves in the main thermocline (depth about $200 \mathrm{~m}$ ) of annual period in MBT (mechanical bathythermograph) data from the tropical North Pacific. From the phase information he inferred that the source of the waves was the eastern boundary. In a similar analysis white (1978) presented evidence from the mid-latitude North Pacific for seasonal fluctuations in the depth of the main thermocline. He demonstrated that the phase of those fluctuations matched the phase of the observed windstress curl; however the observed amplitude was 5 to 10 times that expected from the theory of Veronis and stommel (1956). No explanation of the amplitude mismatch is given by White.

In the North Atlantic the main thermocline is considerably deeper than $200 \mathrm{~m}$ in mid-latitudes $(600-800 \mathrm{~m})$, well removed from any direct solar influence. There are also major sources of deep and bottom water whose formation at the surface at high latitudes and in the Mediterranean Sea occurs seasonally. In the western part of the basin there is an energetic mesoscale eddy field associated with the Gulf stream system. 
In the topographically confined Florida Current whose transport contributes significantly to the Gulf stream, Niiler and Richardson (1973) cite evidence of seasonal fluctuations representing half the total variability of the current and about $10 \%$ of its total transport. Away from the Florida strait, however, no evidence for seasonal variability below the main thermocline has been cited in the western North Atlantic. The energetic eddy field associated with the Gulf stream will tend to mask the relatively weak annual signal expected in the interior, necessitating long observational records to detect the signal unambiguously. Wunsch (1972) computed spectra of temperature and dynamic height anomaly using the 13 years of PANULIRUS hydrographic data taken semi-monthly at a single location near Bermuda. He found a pronounced annual peak in temperature at $10 \mathrm{~m}$ depth, a less pronounced peak at $100 \mathrm{~m}$ and none at $800 \mathrm{~m}$. He also found annual peaks in the dynamic height anomaly spectra, but all the energy at that frequency comes from depths shallower than $200 \mathrm{~m}$. Thus, even in a very long single record in the western North Atlantic the seasonal signal, if it exists, has not been extracted from the noise. Nevertheless, the available meteorological observations in the North Atlantic indicate that there is a strong annual cycle to the windstress curl, with large horizontal scales (Bunker and Worthington, 1976) so that some annual response in the ocean interior is expected. It is anticipated that the observation of that annual signal is most likely to be made in the eastern basin, away from the energetic Gulf stream system, and particularly in a region of relatively weak stratification near the eastern boundary. As discussed briefly in the introduction, the boundary results in free waves as part of the solution to the forced problem; the weaker the stratification, the shorter the horizontal scale 
of the free waves, which in turn results in larger displacements.

Bryan and Ripa (1978) constructed a model of the oceanic response to large scale continuous wind forcing at low frequencies, for the special case of a flat bottomed ocean with depth variable stratification and a single meridional barrier at the eastern edge. They applied their model to the mid-latitude North Pacific at frequencies corresponding to periods of 3 and 6 years, using an idealization of the observed windstress, and appropriate oceanic parameters. They calculated the resultant scales of the dominant free modes and estimated the apparent vertical propagation for comparison with observational estimates of the vertical propagation of low frequency temperature anomaly structures in the North Pacific. Bryan and Ripa made no attempt to calculate the amplitude of the response, although they discuss the phase of the solution at length. Their approach is appropriate to the present work, and their model is discussed in detail when it is applied, with some modification and extension, to the three years of CTD data from the eastern North Atlantic, in Chapter 3.

The region of the eastern North Atlantic in which the data used in this work were collected has no major sub-surface current systems, with the possible exception of a weak (.0I to $.05 \mathrm{~m} \mathrm{sec}^{-1}$ ) poleward eastern boundary current of 60 to $250 \mathrm{~km}$ width and undetermined depth range. Away from the coast the available direct current measurements indicate very low mean flows ( $.01 \mathrm{~m} \mathrm{sec}^{-1}$ ) (Swallow et al., 1977). There is no evidence for a strongly energetic eddy field as is found in the western basin, although isolated eddy-like features have been observed (Swallow, 1969 and Gascard, 1980). with velocities of .10-.20 $\mathrm{m} \mathrm{sec}^{-1}$ associated with the cyclonic flow, and some indication of slow westward 
drift, on the order of $.02 \mathrm{~m} \mathrm{sec}^{-1}$.

There are a number of water. masses, as distinguished by temperaturesalinity relationship, found in this region. Of primary interest to this work are the water masses found between the main thermocline and roughly $2000 \mathrm{~m}$, which is the deepest that the CTD stations penetrate. Those water masses are Mediterranean Outflow Water (MOW), which is found in the western Mediterranean Sea and is characterized by high salinity as a function of temperature compared to: North Atlantic Deep Water, and Labrador sea Water, formed in the Labrador Basin and similarly characterized by low salinity. The interaction of these two water masses within the area studied results in strong mean lateral gradients of salinity anomaly. (defined as the difference between the observed salinity and a reference salinity). Other work has been done which exploits these strong lateral (i.e., isopycnal) gradients of salinity anomaly, notably Needler and Heath (1975) and Katz (1970). Of principal interest here is the identification in both cases of strong lateral gradients of a suitably defined salinity anomaly.

In order to study the lateral distribution of any tracer (such as salinity anomaly) it is necessary to define the density surfaces to be used. Density surfaces rather than horizontal surfaces are used since a minimum of work is performed in moving a parcel along a surface of constant density. Ideally what is sought are those level surfaces which are connected to the observed density field by adiabatic displacements at each depth. Orily recently has a computational technique been developed for determining these implicit adiabatically leveled surfaces (Bray and Fofonoff, 1980). That technique is described in section 4 of this chapter. 
Montgomery (1938) in his isentropic analysis of the southern North Atlantic used $\sigma_{t}$ surfaces as an approximation to constant potential density surfaces. (If a parcel of water at pressure $p$ has temperature $T$ and salinity $S$, then $\sigma_{t}$ is given by

$$
\sigma_{t}(p, T, S)=\left(\rho\left(p_{a}, T, S\right)-I\right) \times 10^{3}
$$

$\rho$ the density, evaluated at atmospheric pressure $\left.p_{a} \cdot\right)$ Surfaces of constant $\sigma_{t}$ are a reasonably good approximaton to constant potential density surfaces for observations shallower than $1000 \mathrm{~m}$. A better approximation is a surface of constant $\sigma_{\theta}$, for which the effect of the adiabatic change of temperature with pressure has been corrected. If $\theta\left(p, p_{r}\right)$ is the potential temperature at pressure $p$ referred to pressure $p_{r}$ (Fofonoff, 1977):

$$
\theta\left(p, p_{x}\right)=T(p)+\int_{p}^{p} \Gamma d p
$$

( $\Gamma$ the adiabatic lapse rate $\left.\left(\frac{\partial T}{\partial p}\right)_{a}\right)$

then $\sigma_{\theta}$ is defined by

$$
\sigma_{\theta}(p, T, s)=\left(p\left(p_{a}, \theta\left(p, p_{a}\right), s\right)-1\right) \times 10^{3}
$$

$\mathrm{p}_{\mathrm{a}}$. the atmospheric pressure. However, in the deep water, $\sigma_{\theta}$ surfaces are no longer a good approximation to constant potential density surfaces (due to large changes in the coefficient of thermal expansion with pressure), so that some pressure other than atmospheric pressure must be used as the reference. Reid and Lynn (1971) used $\sigma_{1}, \sigma_{2}$ and $\sigma_{4}$, 
corresponding to reference pressures ${ }^{2} p_{r}$ of 1000,2000 and $4000 \mathrm{db}$. Thus $\sigma_{p_{r}}$ is given by

$$
\sigma_{p_{r}}(p, T, s)=\left(\rho\left(p_{r}, \theta\left(p, p_{r}\right), s\right)-1\right) \times 10^{3}
$$

The adiabatic leveling technique uses a different reference pressure $P_{r}$ at each depth for which a surface is calculated, with the additional constraint that mass is conserved in the leveling process. The questions of the appropriate $p_{r}$ for a given depth range and of transition from one $p_{r}$ to another are thus separated.

The adiabatically leveled reference state also represents the adiabatic minimum in potential energy: that is, no further energy can be extracted from the system by adiabatic processes. The potential energy in the observed field which is actually available for conversion to kinetic energy (available potential energy or APE) is the difference in the horizontally averaged total potential energy and the adiabatic minimum of potential energy. Lorenz (1955) developed this concept in application to the atmosphere. If the atmosphere is taken to behave as an ideal gas, the total potential energy and internal energy are proportional; consequently Lorenz defines APE as the difference in enthalpy (the sum of potential and internal energies) between the observed and reference states. In a compressible fluid the vertical displacement of an isopycnal may be accompanied by changes in volume due to differential compressibility. (Compressibility is a function of pressure and temperature.) In the atmosphere those volume changes are large cone third of the total enthalpy change) and act to reduce the potential energy. In the ocean they are small and generally act to increase the potential energy. As will be 
demonstrated in some detail in the next section, these changes in potential energy result in very small changes in APE, since most of the change is not available for conversion to kinetic energy at all, but must go into changing the reference field.

A difficulty of definition then arises as to what represents the true APE. It is neither the enthalpy difference nor the change in potential energy which includes all of the change due to compressibility. In the following section a system of nomenclature is proposed to distinguish the different estimates of $\mathrm{APE}_{j}$ a simple example is discussed which illustrates the problem in more detail, and the computational technique for identifying the adiabatically leveled reference densicy fjeld is presented. 


\subsection{Potential Energy}

(The discussion in this section follows the similar discussion in Bray and Fofonoff, 1980).

The total potential energy contained in a column of seawater of unit cross-sectional area relative to a reference level $z_{r}$ is given by

$$
E=\int_{z_{r}}^{z_{s}} \rho\left(\phi-\phi_{r}\right) d z
$$

with $\rho$ the density, $\phi$ the geopotential and $z_{s}$ the height of the surface of the ocean. If the fluid is in hydrostatic balance, (I.I) may be rewritten in terms of pressure:

$$
\text { since } \quad E=\frac{1}{g} \int_{p_{S}}^{p_{r}}\left(\phi-\phi_{r}\right) d p
$$

-

$$
d p=-\rho g d z=-\rho d \phi
$$

The geopotential $\phi$ may also be written in terms of pressure:

$$
\phi-\phi_{r}=\int_{p}^{p_{r}} \alpha d p^{\prime}
$$

with $\alpha$ the specific volume, $\alpha=1 / \rho$. Thus, in terms of pressure and specific volume the total potential energy is

$$
E=\int_{p_{S}}^{p_{r}} \frac{1}{g} \int_{p}^{p_{r}} \alpha d p^{\prime} d p
$$


Lorenz (1955), in his studies of the general circulation of the atmosphere, pointed out that the total potential energy has little dynamical relevance, since only a very small percentage is available for conversion to kinetic energy; specifically, that which is in excess of the adiabatic minimum. That excess, for a column of unit cross-section is

$$
\Delta E=\int_{p_{s}}^{p_{r}} \frac{1}{g} \int_{p}^{p_{r}}\left(\alpha_{i}-\alpha_{f}\right) d p^{\prime} d p
$$

with $\alpha_{f}$ the reference (adiabatically leveled) specific volume, a function only of pressure, and $\alpha_{i}$ the observed specific volume. $\Delta E$ can be either negative or positive, depending upon the sign of $\alpha_{i}-\alpha_{f}$. However there is a net positive storage of available energy averaged over a volume for which mass is conserved during leveling. The net storage is the total available gravitational potential energy $\overline{\Delta \mathrm{E}}$, averaged horizontally over an area $\mathrm{A}$ :

$$
\overline{\Delta \mathrm{E}}=\frac{1}{\mathrm{~A}} \iint_{\mathrm{A}} \Delta \mathrm{E} d \mathrm{~A}=\int_{\mathrm{p}_{S}}^{\mathrm{p}_{\mathrm{S}}} \frac{1}{\bar{g}} \int_{\mathrm{p}}^{\mathrm{p}_{\mathrm{f}}}\left(\overline{\alpha_{i}}-\alpha_{f}\right) d \mathrm{p}^{\prime} d \mathrm{p}
$$

Contributions to the total $\overline{\triangle E}$ from within the column are identified as the total available gravitational potential energy (TGPE):

$$
\left.\operatorname{TGPE}(p)=\int_{p}^{p_{r}} \frac{1}{g} \int_{p}^{p_{r}} \overline{\left(\alpha_{i}\right.}-\alpha_{f}\right) d p^{-1} d p^{\prime}
$$

with units of potential energy per unit area $\left(\mathrm{J} \mathrm{m}^{-2}\right)$ and contributions to TGPE at each pressure: 


$$
\operatorname{GPE}(p)=g \frac{d T G P E}{d p}=\int_{p}^{p_{x}}\left(\bar{\alpha}_{i}-\alpha_{f}\right) d p^{-}
$$

with units of potential energy per unit mass $\left(\mathrm{J} \mathrm{kg}^{-1}\right)$.

As an illustration of the relationship between the gravitational potential energy and the thermodynamic energies of the system consider the following simple example. A rigorous mathematical derivation is found later in section 3. Take a volume of seawater of length and width I and infinite vertical extent, with specific volume initially level. Next, displace two columns of fluid of unit mass adiabatically a distance (in pressure units) $\pi$ : one upward and one downward, thereby conserving mass at each pressure. If the reference density varies linearly with depth, no net work is done (except small changes of internal energy, which are discussed later). That is, the horizontal. average of $\alpha_{i}-\alpha_{f}$ is zero since the perturbation of the upward displacement results in the same change of volume as that of the downward displacement, unless there are gradients of compressibility present, in which case conversions of internal energy to potential energy are possible. However, if density is a non-linear function of depth, the upward displacement results in a different change of volume than the downward displacement; there is a net change in specific volume averaged over $L$, and net work is performed. A series of schematics relating to this example are found in Fig. 1.I. Although the motion is presumed to extend throughout the fluid the representation of the observed and reference surfaces as functions of pressure and specific volume are shown for a single level. In Fig. 1.la the level 
Figure 1.J. Schematic of displacements in an initially level steric field. See text for detailed explanation. 

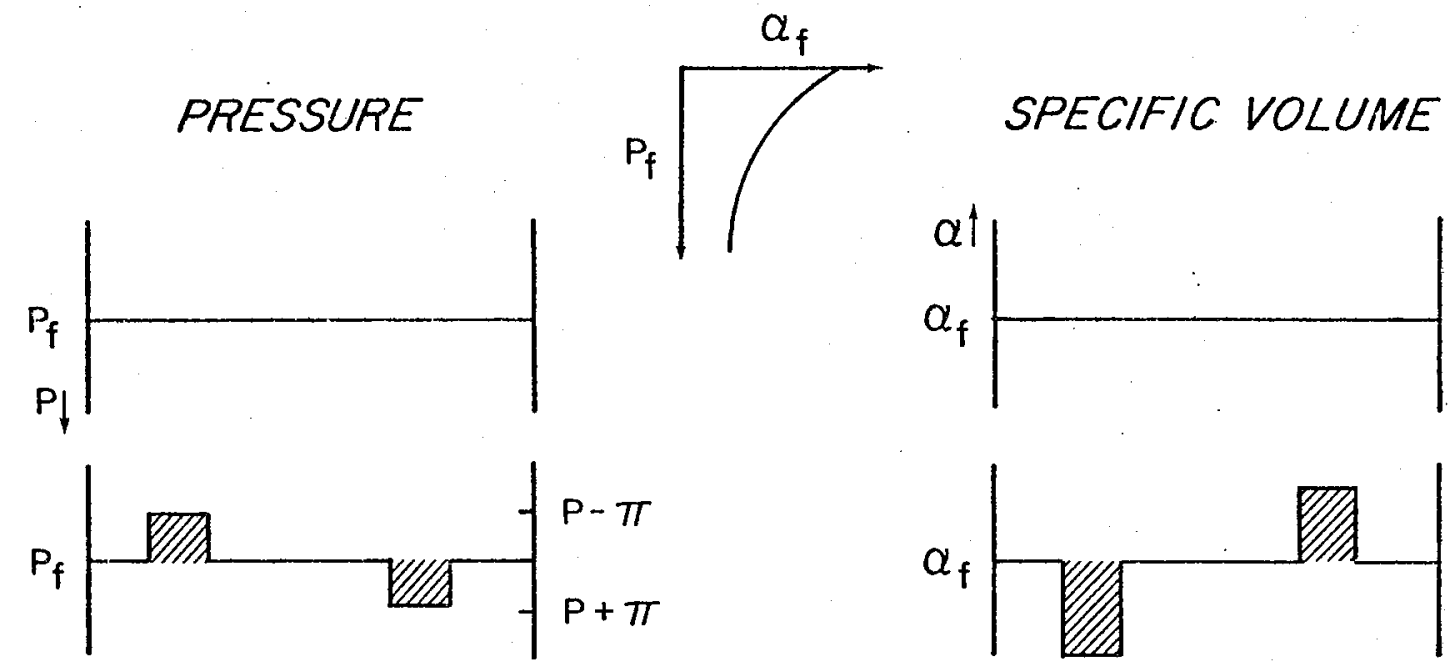

(A)

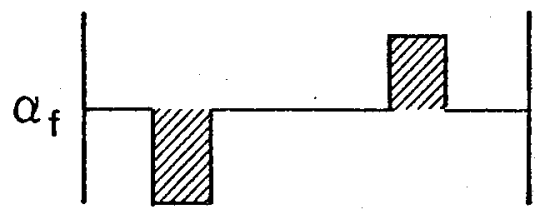

(B)
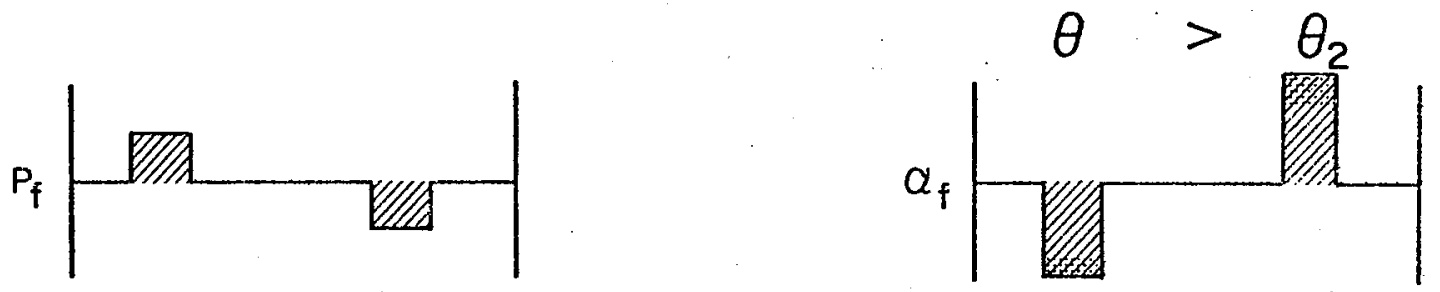

(C)
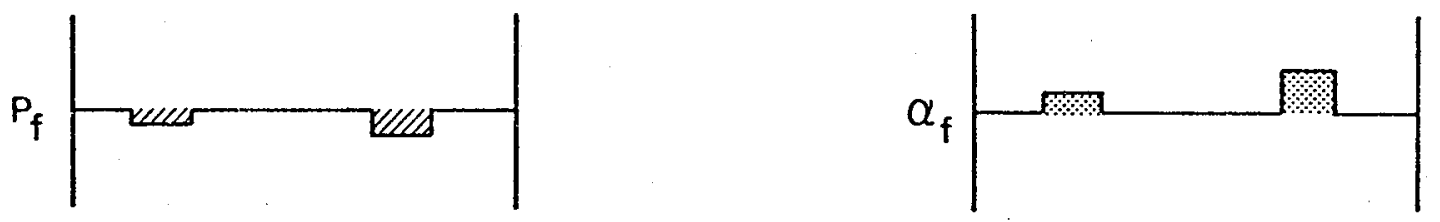

(D)
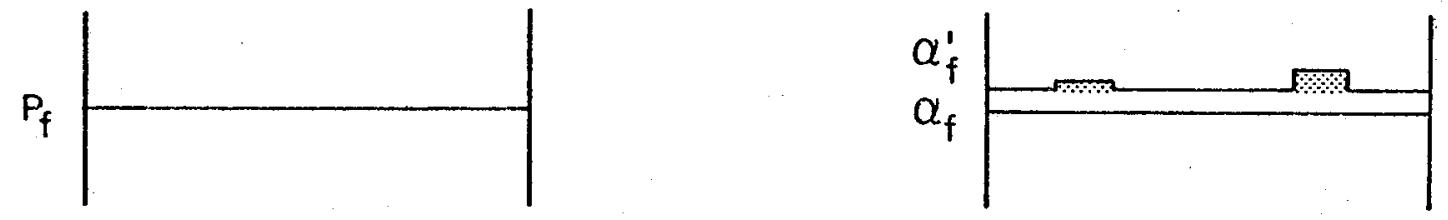

(E) 
fileld is shown. In Fig. 1.16 the level field is perturbed by displacements $\pi$. The amount of work done can be estimated as follows: the change in volume at pressure $p_{f}$ for the upward displaced (-T) column is given approximately by

$$
\Delta \alpha_{\mathrm{U}} \cong-\pi \frac{d \alpha}{d p}\left(p_{f}+\pi\right)
$$

whereas the change in volume corresponding to the downward displacement is:

$$
\Delta \alpha \stackrel{\cong}{\cong} \pi \frac{d \alpha}{d p}\left(p_{f}-\pi\right)
$$

expanding $\frac{d \alpha}{d p}$ about $p_{f}$, the average change in volume $\overline{\Delta \alpha}$ at each level, per unit mass, is:

$$
\begin{aligned}
\Delta \alpha & \cong \frac{-\pi}{4}\left[\frac{d \alpha}{d p}\left(p_{f}\right)+\left.\frac{d}{d p}\left(\frac{d \alpha}{d p}\right)\right|_{p_{f}} \pi-\frac{d \alpha}{d p}\left(p_{f}\right)+\left.\frac{d}{d p}\left(\frac{d \alpha}{d p}\right)\right|_{p_{f}} \cdot \pi\right] \\
& =\frac{-\pi}{2} \frac{d}{d p}\left(\frac{d \alpha}{d p}\right)
\end{aligned}
$$

Integrating $\overline{\Delta \alpha}$ over pressure as an estimate of APE for this simple case:

$$
\operatorname{APE}\left(p_{f}\right)-\left.\frac{\pi^{2}}{2} \frac{d \alpha}{d p}\right|_{p_{r}}=-\frac{\pi^{2}}{2} \frac{d \alpha}{d p}\left(p_{f}\right)
$$

The right hand side of (1.6) is greater than or equal to zero for a stably stratified fluid. It will be recognized as the Boussinesq 
approximation to APE per unit mass, and represents the contribution to the gravitational potential energy from local changes of the mass. field relative to pressure surfaces with no other change resulting in the mass field globally.

The small changes in internal energy mentioned earlier occur if there are gradients of compressibility as a function of vertical or horizontal position, since the adiabatic displacement at two different positions in space will cause the two parcels involved to compress (or expand) differentially, resulting in an additional change in the horizontally averaged specific volume (Fig. 1.IC). The sign of that avexage change in volume depends upon the spatial gradients of compressibility. Compressibility is primarily a function of temperature (with colder water more compressible), so that these volume changes may be thought of as conversions between internal and potential energy. That conversion process is entirely separate from the local changes of mass relative to a pressure (or geopotential) surface. The change in volume due to that conversion causes global changes in the mass field. The change in volume due to compressibility for the upward displacement is, approximately:

$$
\Delta \alpha_{U} \stackrel{i}{=}-K_{1}\left(\theta_{1}, S_{1}, p+\pi\right) \cdot \pi
$$

and for the downward displacement:

$$
\Delta \alpha_{D} \cong K_{2}\left(\theta_{2}, s_{2}, p-\pi\right) \cdot \pi
$$

with $k$ the adiabatic compressibility $\left(\frac{\partial \alpha}{\partial p}\right), s^{\prime} \theta$ potential temper- 
ature and $S$ salinity (see Fig. 1.1c). The contribution to the gravitational potential energy per unit mass is

$$
\Delta \mathrm{APE}=\int_{\mathrm{p}}^{\mathrm{p}_{r}}\left(\kappa_{2}-\kappa_{1}\right) \cdot \pi d \mathrm{p}^{\prime}
$$

due to horizontal gradients of compressibility. (A corresponding term for vertical gradients in the general case is derived as part of the rigorous dexivation given later in this section.)

If the displaced columns are now moved back to their original positions (a distance $\pi$ ), which corresponds to leveling the observed field, the mass imbalance caused by conversion of internal to potential energy becomes obvious (Fig. 1.Id). Although the figure illustrates only one level, there are corresponding imbalances at all levels. If the specific volume pictured in Fig. 1.ld is taken to be the new 'observed' field, $\alpha_{f}$ is no longer the adiabatic minimum at $p_{f}$. Therefore, in order to estimate how much of the energy given by $(1.7)$ is actually available for conversion to kinetic energy, the field in Fig. 1.ld must be leveled, and deviations from the new reference $\alpha_{f}$ calculated (Fig. 1.le). The amount actually available is a small portion of that calculated in (1.7) since most of the energy must go into changing the reference level $\alpha_{f^{*}}$ The energy given in (1.7) is itself a small correction to the Boussinesq APE (1.6), or order < 15\% for most oceanic applications.

If, rather than considering gravitational potential energy alone. the sum of potential plus internal energies (enthalpy) is considered, the conversions between internal and potential energy will not appear explicitly. (Lorenz, 1955 and Reid, et al., 1980 both define APE as the 
difference in enthalpy between the observed state and the adiabatic minimum.) Since the conversion terms are effectively very small, using enthalpy rather than gravitational potential energy will result in a good approximation to the true APE. In order to use the difference in enthalpy, one must either determine from observations the reference enthalpy field or else use an expansion about the displacement $\pi$ to evaluate the change in enthalpy between the observed and reference $\therefore$. fields. The first approach presents some practical difficulties, but could, in principle, be used. The second approach is satisfactory unless the displacements are large, in which case higher order terms in the expansion must be included. In that case a better approximation to the true APE is the gravitational potential energy, which can be evaluated exactly, with the small conversion terms like (1.7), whose errors for large displacement are also small, subtracted out. In order to make the rigorous thermodynamic argument which corresponds to the example just given, a few definitions must be made. In the interests of brevity the different estimates of APE are given abbreviations as well:

a. Available Gravitational Potential Energy (GPE)

$$
\text { GPE (per unit mass) }=\int_{p}^{p_{r}}\left(\overline{\alpha_{i}}-\alpha_{f}\right) d p^{\prime}
$$

(with $\alpha_{i}$ and $\alpha_{f}$ the initial and reference specific volumes, respectively, $p_{x}$ the reference pressure, and the overbar indicating horizontal average). 
b. Total Available Gravitational Potential Energy (TGPE):

$T G P E$ (per unit area) $=\int_{p}^{p_{r}} \frac{1}{g} \int_{p}^{p_{r}}\left(\bar{\alpha}_{i}-\alpha_{f}\right) d p^{\prime \prime} d p^{\prime}$

c. The Boussinesq Approximation to $\mathrm{APE}\left(\mathrm{APE}_{B}\right)$ :

$\mathrm{APE}_{\mathrm{B}}$ (per unit mass) $=\frac{1}{2} \overline{\alpha_{\mathrm{p}}^{*} \cdot \pi^{2}}=\frac{1}{2} \cdot \overline{\mathrm{N}^{2} \cdot \zeta^{2}}$

(with $\pi$ the displacement in decibars, $\underset{p}{\alpha}$ the vertical gradient of specific volume, $\mathrm{N}$ the buoyancy frequency and $\zeta$ the displacement in meters).

d. Available potential energy as defined by Lorenz (1955) and Reid et al. (1980) (HPE) :

HPE (per unit area) $=\frac{1}{g} \int_{p}^{p_{r}}\left(\bar{h}_{i}-h_{f}\right) d p^{\prime}$

( $h_{i}$ and $h_{f}$ the initial and reference specific enthalpies, respectively). The relationships between $\mathrm{GPE}, \mathrm{APE}_{\mathrm{B}}$ and $\mathrm{HPE}$ can be demonstrated most readily by considering perturbation expansions in displacement about the adiabatically leveled reference state. For any state variable $\psi$, the connection between the initial and reference states may be written:

$$
\begin{aligned}
\psi_{i}(p) & =\psi_{f}\left(p-\pi^{\prime}\right)+\int_{p-\pi}^{p}\left(\frac{\partial \psi}{\partial p^{\prime}}\right) d p^{\prime} \\
\pi^{\prime} & =\pi\left(p-\pi^{\prime}\right)
\end{aligned}
$$


(Subscript a indicates differentiation under adiabatic and isentropic conditions; i.e., with salt and entropy held constant.)

The displacement $\pi^{\prime}$ represents the adiabatic displacement of the reference surface at $\mathrm{p}-\pi$; because displacements are a function of pressure, $\pi^{-}$differs from $\pi$, which is the displacement at pressure p. (See Fig. 1.2 for an illustration.)

Expansion about $\pi^{\prime}$ results in the following equation:

$$
\begin{aligned}
& \psi_{i}(p)=\psi_{f}(p)-\frac{d \psi_{f}}{d p} \pi^{\prime}+\frac{1}{2} \frac{d^{2} \psi_{f}}{d p^{2}} \pi^{-2}+\kappa \pi^{-}-\frac{d \kappa}{d p} \pi^{-2}+ \\
& \frac{1}{2}\left(\frac{\partial K}{\partial p_{a}} \pi^{-2}+O\left(\pi^{3}\right)\right.
\end{aligned}
$$

with

$$
\pi^{\prime}=\pi-\frac{d \pi}{d p} \pi^{\prime}+\ldots=\pi-\frac{d \frac{1}{2} \pi^{2}}{d p}+0\left(\pi^{3}\right)
$$

and

(

$$
K(\psi)=\left(\frac{\partial \psi}{\partial p}\right)_{a}
$$

Substituting $(2.6 \mathrm{~b})$ into $(2.6 \mathrm{a})$ and keeping only terms of second order in $\pi$, the change in $\psi$ at $\mathrm{p}$ becomes

$$
\Delta \psi=\psi_{i}(p)-\psi_{f}(p)=-\psi_{p}^{*} \pi+\frac{d}{d p}\left(\frac{1}{2} \psi_{p}^{*} \pi^{2}\right)-\frac{1}{2} \Gamma_{K} \pi^{2}+o\left(\pi^{3}\right)
$$

with

$$
\begin{aligned}
& \psi_{p}^{*}=\frac{d \psi_{f}}{d p}-K(\psi) \\
& \Gamma_{K}(\psi)=\frac{d K}{d p}-\left(\frac{\partial K}{\partial p}\right)
\end{aligned}
$$


Figure 1.2. Schematic of the adiabatic leveling calculation. The initial, observed steric anomaly field (solid lines) is assumed to be formed by vertical displacement of a reference steric anomaly field (dashed lines) that is uniform on pressure surfaces. The observed stexic anomaly $\delta_{i}$ on pressure surface $p_{f}$ is the reference surface $\delta_{f-1}$ displaced adiabatically by $\pi$ from the pressure surface $p_{f-1}$. 


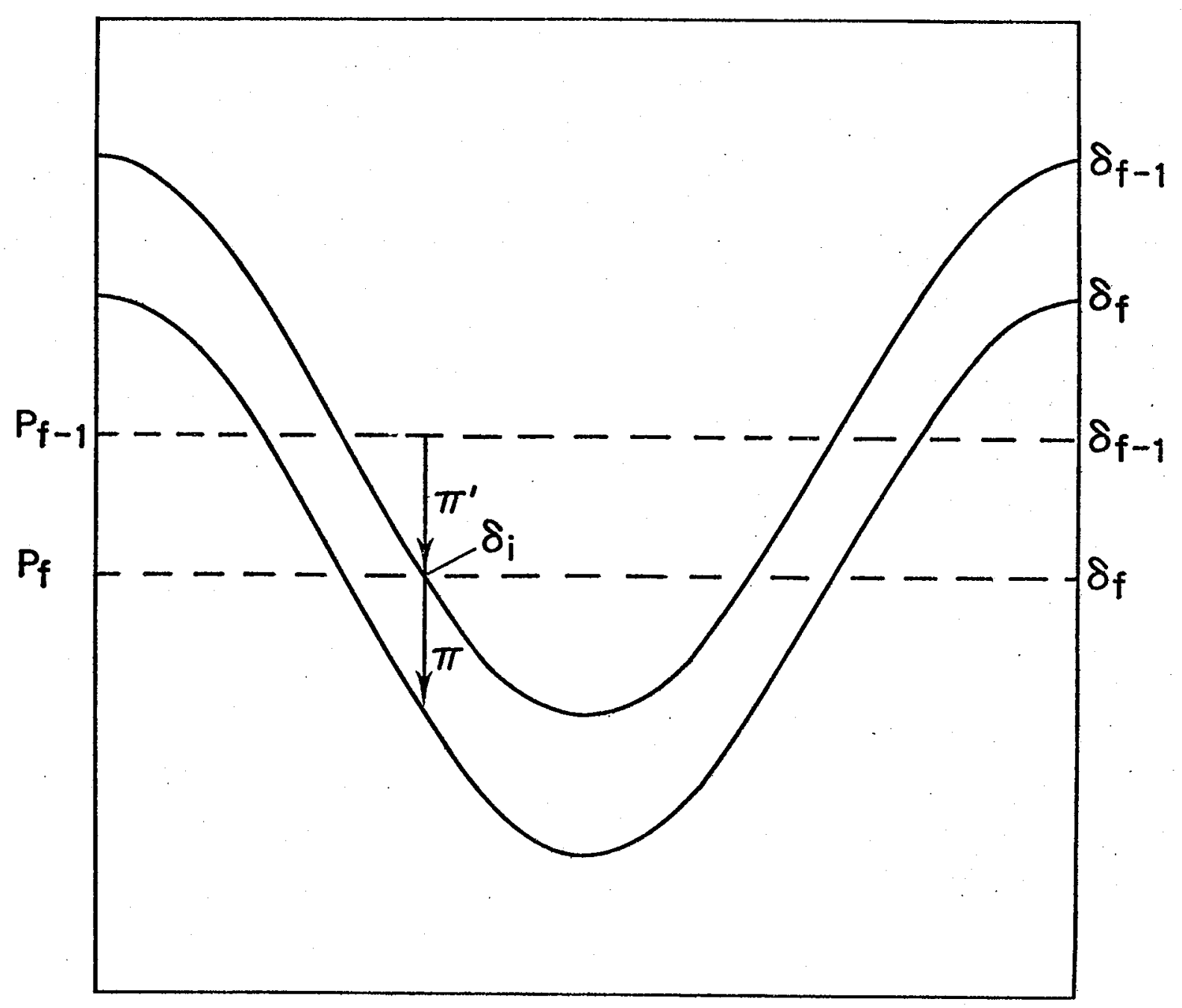


For $\psi=\alpha$, the specific volume, the expansion of GPE is straightforward:

$$
\left.\left.\int_{p}^{p_{r}} \overline{\left(\overline{\alpha_{i}}\right.}-\alpha_{f}\right) d p^{\prime}+\left.\overline{\frac{1}{2} \alpha_{p}^{*} \pi^{2}}\right|_{p_{r}}=\overline{\frac{1}{2} \alpha_{p}^{*} \pi^{2}}-\int_{p}^{p_{r}} \overline{\left(\alpha_{p}^{*} \pi\right.}+\frac{\overline{\Gamma_{k} \pi^{2}}}{2}\right) d p^{\prime}
$$

The first term on the right is the Boussinesq $\mathrm{APE}_{B}$ (the term at $\mathrm{p}_{r}$ is evaluated and used as a boundary condition); the remaining integral term represents conversions of internal to potential energy. Physically, these come about because of the small change in volume which results from interchanging parcels of water with different compressibilities. As described earlier, leveling moves colder (more compressible) water to lower pressure and warmer (less compressible) water to higher pressure. The small change in volume which results causes a shift in the mass field above the location of the leveled surface. The first half of the integral term represents conversions due to horizontal gradients of compressibility: since $d \alpha_{f} / d p$ is not a function of horizontal position, $\overline{\alpha_{p}^{*} \pi}$ reduces to $\overline{-K \pi}$ - the horizontal correlation of adiabatic compressibility and displacement. The second half of the integral term represents changes due to vertical gradients of compressibility.

To understand the relationship between TGPE and HPE, an expansion using $\psi=h$ is suggested:

$$
\Delta h=-h_{p}^{*} \pi+\frac{1}{2} \frac{d}{d p} \cdot \underset{p}{h * \pi^{2}}-\frac{\Gamma_{\dot{k}}(h) \pi^{2}}{2}+O\left(\pi^{3}\right)
$$

and

$$
\Delta e=-e_{p}^{*} \pi+\frac{1}{2} \frac{d}{d p} e_{p}^{*} \pi^{2}-\frac{F K(e) \pi^{2}}{2}+0\left(\pi^{3}\right)
$$


with $e$ the specific internal energy. Using the following definition of enthalpy (Fofonoff, 1962):

$$
d h=T d \eta+\mu d s+\alpha d p=d(\alpha p)+d e
$$

with $T$ absolute temperature, $n$ the specific entropy, and $\mu$ the chemical potential of salinity s) the following expressions may be written in terms of the specific volume $\alpha$ :

$$
\begin{aligned}
& \Gamma_{K}(h)=\frac{d \alpha}{d p}-\left(\frac{\partial \alpha}{\partial p}\right)_{a}=\alpha_{p}^{*} \\
& \Gamma_{K}(e)=\alpha_{p}^{*}-p \Gamma_{K}(\alpha) .
\end{aligned}
$$

Then

$$
\overline{\Delta h-\Delta e}=\overline{-p \alpha_{p}^{*} \pi}+\overline{\frac{1}{2} \frac{d}{d p}\left(p \alpha_{p}^{*} \pi^{2}\right)}-\overline{\frac{p \Gamma_{K}(\alpha) \pi^{2}}{2}}+O\left(\pi^{3}\right)
$$

which, except for a constant of integration, is equivalent to GPE. Thus HPE is the sum of TGPE and any changes of internal energy, to second order in $\pi$. Since it was shown earlier that GPE is the sum of $\mathrm{APE}_{\mathrm{B}}$ and changes of potential energy due to conversions from internal energy, it follows that $g(d H P E / d p)$ and $A P E_{B}$ are equivalent to second order in $\pi$, provided that $\mathrm{APE}_{\mathrm{B}}$ is calculated using the adiabatic displacements from the reference field, rather than the mean field. 
1.4 Procedure for calculating the adiabatically leveled steric field There are two stages in the computational procedure for determining the adiabatically leveled reference field. In the first stage, for each CTD profile, pressure and potential temperature $\theta$ (Fofonoff, 1977)

$$
\theta\left(p, p_{f}\right)=T(p)+\int_{p}^{p_{f}}\left(\frac{\partial T}{\partial p}\right) d p^{\prime}
$$

are fit to nth order : polynomials against potential specific volume (steric) anomaly:

$$
\delta\left\{p, p_{f}\right)=\alpha\left(p_{f}, \theta\left(p, p_{f}\right), S(p)\right)-\alpha(p, 0,35)
$$

The regressions are performed over an interval of pressure $\Delta p$ about $p_{f}$ (so that $\theta$ and $\delta$ are referred adiabatically to $p_{E}$ rather than to atmospheric pressure) for a number of levels. The intervals $\Delta \mathrm{p}$ may overlap.

In the second stage, the coefficients of the pressure polynomials at each level are averaged about a common oxigin $\delta_{m}$ :

$$
\delta_{m}=\frac{I}{M} \sum_{k=1}^{M} \delta_{k}
$$

$\delta_{k}$ the average $\delta$ over $\Delta p$ for station $k$. If the pressure regressions for individual stations at a given level are:

$$
\hat{p}_{k}=\sum_{n=0}^{N} \alpha_{k n}\left(\delta-\delta_{k}\right)^{n}
$$


then the average coefficients (about $\delta_{m}$ ) define the horizontal average of $\hat{p}_{k}\left(\delta_{f}\right)$ :

$$
\frac{1}{M} \sum_{k=1}^{M} \hat{p}_{k}=\frac{1}{M} \sum_{k=1}^{M} \sum_{n=0}^{N} \alpha_{k n}^{\prime}\left(\delta_{f}-\delta_{m}\right)^{n}
$$

with

$$
\alpha_{k n}^{\prime}=\sum_{r=0}^{N-n} \alpha_{k, n+r} \frac{(n+r) !}{n ! r !}\left(\delta_{m}-\delta_{k}\right)^{r} .
$$

The constraint of mass conservation during leveling requires the initial mass $p_{i} / g$ and the final mass $p_{f} / g$ above the surface corresponding to $\delta_{f}$ to be the same, or

$$
p_{f}=\frac{1}{M} \sum_{k=1}^{M} \hat{p}_{k}\left(\delta_{f}\right)=\frac{1}{M} \sum_{k=1}^{M} \sum_{n=0}^{N} \alpha_{k n}^{\prime}\left(\delta_{f}-\delta_{m}\right)^{n} .
$$

This equation may be inverted to obtain $\delta_{f^{-}}$once $\delta_{f}$ is determined, the displacements $\pi$, and the initial and final potential temperatures $\theta_{i k}$ and $\theta_{f}$ are given by

$$
\pi_{k}=p_{i k}-p_{f}=\sum_{n=0}^{N} \alpha_{k n}\left(\delta_{f}-\delta_{k}\right)^{n}-p_{f}
$$

and

$$
\begin{aligned}
\theta_{i k} & =\sum_{n=0}^{N} \beta_{k n}\left(\delta_{i}-\delta_{k}\right)^{n} \\
\theta_{f} & =\sum_{n=0}^{N} \beta_{k n}\left(\delta_{f}-\delta_{k}\right)^{n}
\end{aligned}
$$


with $\beta_{\mathrm{kn}}$ the potential temperature regression coefficients for a given station. Note that salinity is determined implicitly.

In order to illustrate in a simple way what the computational procedure actually does, consider the case $N=I$, i.e., specific volume a linear function of depth, over an interval $\Delta p$ centered about $\mathrm{p}_{\mathrm{f}}$. If several stations are included, all with $\delta$ varying linearly with depth, but with different slopes and different average values of $\delta$ (referred adiabatically to $p_{f}$ ), then the coefficients $\alpha_{k 0}$ (which are equal to $p_{f}$ ) are the same for all k, but $\alpha_{k l}$ and $\delta_{k}$ differ from station to station. The corrected coefficients $\alpha_{\mathrm{kn}}^{\prime}$ for averaging are (eq. 1.21):

$$
\begin{aligned}
& \alpha_{k 0}^{\prime}=\alpha_{k 0}+\alpha_{k I}\left(\delta_{m}-\delta_{k}\right) \\
& \alpha_{k I}^{\prime}=\alpha_{k I} .
\end{aligned}
$$

Averaging over all $\mathrm{k}$, subject to the mass conservation constraint (1.22)

$$
p_{f}=\alpha_{0}+\overline{\alpha_{k I}\left(\delta_{m}-\delta_{k}\right)}+\overline{\alpha_{k l}}\left(\delta_{f}-\delta_{m}\right)
$$

or

$$
\delta_{f}=\delta_{m}-\frac{\overline{\alpha_{k 1}\left(\delta_{m}-\delta_{k}\right)}}{\overline{\alpha_{k 1}}}
$$

(If $\alpha_{k l}$ is the same for all $k$, then

$$
\delta_{f}=\delta_{m} .
$$


For that simple case, $\delta_{f}$ is just the average of $\delta$ over $\Delta p$ and all stations.)

The value of $\delta$ at $p_{f}$ in the observed field $\left(\delta_{i k}\right)$ is just $\delta_{k}$; the contribution to GPE from $p_{E}$ is

$$
\begin{aligned}
\frac{d G P E}{d p} & =\overline{\delta_{i}}\left(p_{f}\right)-\delta_{f}\left(p_{f}\right)=\bar{\delta}_{k}-\delta_{m}+\overline{\alpha_{k l}\left(\delta_{m}-\delta_{k}\right)} / \alpha_{k l} \\
& =\overline{\alpha_{k l}\left(\delta_{m}-\delta_{k}\right) / \alpha_{k l}} .
\end{aligned}
$$

If $\alpha_{k l}$ is a constant for all $k$, the contribution to GPE is identically zero (as expected from the arguments forwarded in the discussion of potential energy). Notice that the compressibility effects have been included by referring the steric anomaly $\delta$ to $\mathrm{p}_{f^{-}}$The adiabatic displacements $\pi_{k}$ are the difference between $p_{f}$ (the pressure corresponding to $\delta_{f}$ in the reference $f i e l d$ ) and $p_{i k}$ (the pressure corresponding to $\delta_{f}$ in the observed field at station $\mathrm{k}$ ):

$$
p_{i k}=\alpha_{k 0}+\alpha_{k l}\left(\delta_{f}-\delta_{k}\right)
$$

so that

$$
\pi_{k}=\alpha_{k I}\left(\delta_{f}-\delta_{k}\right)
$$

Again, for the simple case $\alpha_{k l}$ a constant $\alpha_{1}$

$$
\pi_{k}=\alpha_{1}\left(\delta_{m}-\delta_{k}\right)
$$


The coefficient $\alpha_{1}$ is $\left(\frac{\mathrm{d} \delta}{\mathrm{dp}}\right)^{-1} ; \pi_{k}$ is then the adiabatic generalization (since $\delta$ is referred adiabatically to $p_{f}$ ) of the usual Boussinesq displacement $\pi_{k}^{*}$ :

$$
\pi_{k}^{*}=\frac{\alpha_{k}-\bar{\alpha}}{\frac{d \alpha}{d p}}
$$

In the general case of arbitrary $N$, the higher order polynomial regressions resolve the vertical structure of $\delta$ within $\Delta \mathrm{p}$, and thereby provide an accurate weighting of the specific volume information as a function of pressure for the specialized averaging and subsequent inversion which results in the reference steric anomaly at a single pressure.

Finally, the dynamic height $\Delta \mathrm{D}_{\mathrm{k}}$, now defined using $\alpha_{\mathrm{f}}$ rather than $\alpha(p, 0,35)$ (Fofonoff, 1962), and GPE are calculated by numerical integration of

$$
\Delta D_{k}=\int_{0}^{p}\left(\delta_{i k}-\delta_{f}\right) d p^{\prime}
$$

and

$$
\mathrm{GPE}=\int_{p}^{\mathrm{p} r}\left(\overline{\delta_{i k}}-\delta_{f}\right) d \mathrm{p}^{\prime}
$$

Discussion of the errors resulting from measurement, finestructure and numerical exrors is deferred to Chapter 2. 
Chapter 2

\subsection{Introduction}

The observation of low frequency signals in the ocean requires a combination of long time series and adequate spatial coverage. Over the last twenty years the technology for obtaining long current meter records in the deep ocean has progressed sufficiently that there are now available 2 to 3 year records which are nearly continuous. However, these measurements are necessarily limited in their horizontal coverage, due to the cost of instrumentation. An alternative approach to the problem is the use of hydrographic measurements (temperature, salinity and pressure) which have better spatial coverage both vertically and horizontally, but are not continuous in time at any level. From repeated hydrographic measurements within a given area over a sufficiently long period, however, time series of horizontally averaged quantities can be constructed, and the structure of low frequency motions can be studied. For seasonal signals this requires a minimum of four samples per year taken over a period of at least a year, and over a fairly extensive area whose dimensions are determined by the dominant length scales of the seasonal signal. Anticipating the results of Chapter 3 , the minimum wavelength is about $400 \mathrm{~km}$. The horizontal resolution of the data should be less than a quarter of the dominant wavelength, or station spacing of no more than about $100 \mathrm{~km}$ for the minimum wavelength.

One set of hydrographic data which meets these requirements is a group of approximately 600 stations occupied by French investigators (Fruchaud, 1975; Fruchaud, et al., 1976a, 1976b) over a period of three 
years in the Bay of Biscay off the western coast of France. The data consists of eleven cruises spaced roughly three months apart, of which eight used CTD (conductivity, temperature, depth) instruments, and three used STD (salinity, temperature, depth) instruments. The data were obtained through the United States National Oceanographic Data Center, in the format in which it was issued by the French Organization Bureau National de Données Océaniques. The cruises are summarized in Table 2.1. The majoxity of stations were occupied in a region bounded by $z^{\circ}$ to $12^{\circ} \mathrm{W}$ and $43^{\circ}$ to $48^{\circ} \mathrm{N}$ (see Fig. 2.1 and Table 2.1). For the purpose of comparison, only stations within those limits were used in the calculation of lateral fields. The bottom topography of that region (Fig. 2.1) is predominantly abyssal plain, although a sharp shelf break and steep continental slope mark the eastern, southern, and angled northern boundaries. The simplicity of the topography does not extend much beyond the western boundary of the smaller region chosen for study.

The original version of the data is an uneven pressure series of approximately one decibar resolution. Corrections for calibration errors were made by the acquisition group (Fruchaud, 1975). The original CTD data was converted to salinity, temperature and pressure by the author, using the algorithm from Fofonoff, Hayes and Millard (1974). The pressure, temperature and salinity data were then pressure sorted and smoothed into even 10 decibar series in preparation for the adiabatic leveling calculation. 
TABLE 2.1

Description of Phyyas Cruises

CRUISE

1. Phygas 22

2. Phygas 23

3. Phygas 24

4. Phygas 31

5. Phygas 32

6. Phygas 33

7. Phygas 34

8. Phygas 41

9. Phygas 42

10. Phygas 43

11. Phygas 44

$$
\text { (from } \frac{\mathrm{DAY}^{\mathrm{l}}}{\mathrm{I} \operatorname{Jan} 1972 \text { ) }}
$$

$$
124-135
$$$$
235-242
$$$$
294-307
$$$$
383-393
$$$$
480-492
$$$$
554-567
$$$$
627-641
$$$$
780-794
$$$$
908 \text { - } 920
$$$$
982-994
$$$$
1076-1097
$$

DEPT
1200
1250
1250
1750
1750
1700
1300
1750
1800
1750
1750

1750
INSTRUMENT
TYPE

CTD

CTD

CTD

CTD

CTD

CTD

STD

STD

CTD

STD

CTD
No. ${ }^{5}$

STATIONS

31 (15)

70 (21)

68 (24)

58 (37)

60 (38)

44 (33)

$76 \quad(50)$

$54(41)$

$62(48)$

39 (39)

47 (44)

1 Covers the period during which stations (within the small box) which extend deeper than $1000 \mathrm{db}$ were taken.

2 Maximum depth common to at least 10 stations, after regressions, for the restricted region (Maximum depth before regressions: add 250 dbar:)

3 Total number of stations. In parenthses: number of stations within the restricted region $\left(2^{\circ}-12^{\circ} \mathrm{W}, 43^{\circ}-48^{\circ} \mathrm{N}\right)$ deeper than $250 \mathrm{~m}$. 
Figure 2.1 General location of Bay of Biscay stations, showing bottom topography. The large box encloses all stations taken during the tree year period; the smaller box outlines area of greatest concentration of stations. The center of the smaller box, referred to in the text as the origin, is marked with a cross. 


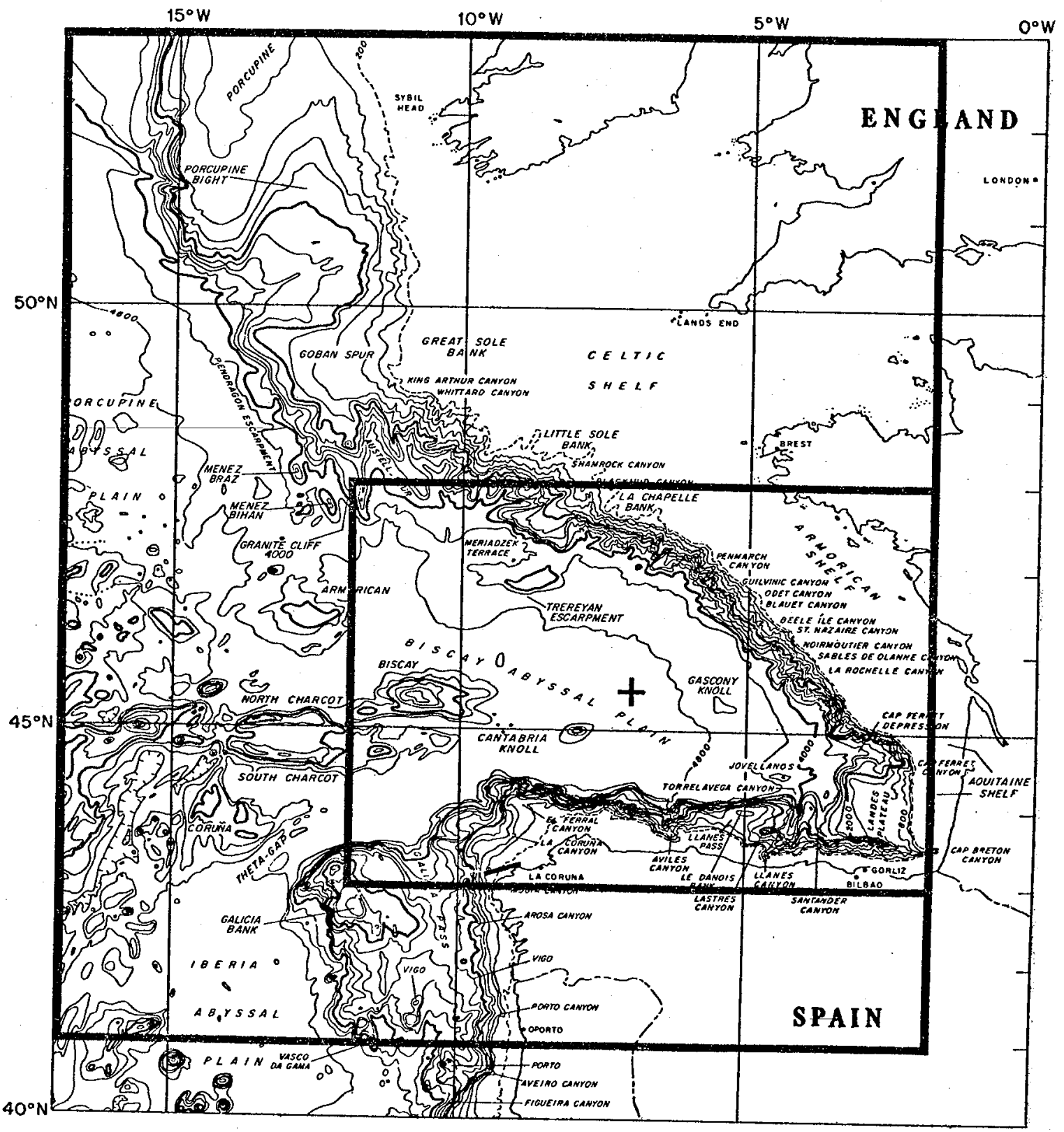




\subsection{Application of the adiabatic leveling technique}

The variable parameters used in the initial regressions $p(\delta)$,

$\theta(\delta)$ are summarized in Table 2.3, with the values which were used in all eleven cruises. This initial calculation was performed for all stations in all cruises. The horizontal averaging and determination of the reference specific volume field, the displacements, and the potential temperature (hence implicitly salinity) on the reference and observed specific volume surfaces was performed separately for the subset of stations within $2^{\circ}-12^{\circ} \mathrm{W}, 43^{\circ}-48^{\circ} \mathrm{N}$, for each cruise; the results which follow are only those from this restricted region. The number of stations in the restricted region for each cruise is given in Table 2.I.

There are several sources of uncertainty in the adiabatic leveling calculation, resulting from measurement, finestructure, and numerical errors. Methods for calculating the corresponding errors in derived quantities are described below. The errors themselves will be found plotted in various figures and listed in tables as noted. For a complete discussion of the relative errors in the different estimates of APE, the reader is refexred to Bray and Fofonoff: (1980). Numerical erxors are only important in calculations involving numerical integration (GPE, TGPE, HPE, dynamic height), and result from inadequate resolution of vertical struature. A method for determining the correct vertical resolution of the adiabatic leveling technique in order that integration errors are made smaller than non-reducible errors is described in detail also in Bray and Fofonoff (1980).

This discussion concentrates on the effects of measurement and finestructure errors on derived quantities such as vertical displacement, GPE, and maps of salinity or temperature on leveled steric surfaces. By 
TABLE 2.2

Calibration errors: CTD/STD

$\begin{array}{lll}\text { Salinity } & \text { Temperature } & \text { Pressure } \\ \pm .010 \mathrm{ppt} & \pm .010^{\circ} \mathrm{C} & \pm 5 \mathrm{db}\end{array}$


TABLE 2.3

\begin{tabular}{cccc}
\multicolumn{2}{c}{ Initial Calculation } & Regression Parameters \\
$\mathrm{P}_{\mathrm{f}}$ & step & $\Delta \mathrm{P}$ & $\mathrm{N}$ \\
0,200 & $(50)$ & 250 & 6 \\
250,350 & $(50)$ & 400 & 5 \\
400,450 & $(50)$ & 500 & 5 \\
500,1100 & $(50)$ & 450 & 5 \\
1150,2000 & $(50)$ & 500 & 4
\end{tabular}


measurement error is meant the uncertainty in the measured values of. pressure, temperature, and salinity (or conductivity) which results from uncorrected calibration errors (quantization noise is ignored) and which will be taken to be randomly distributed from one station to the next, but constant for a given station; random finestructure errors are the fluctuations caused by real variability of the field which occurs on smaller scales than those over which the calculations are performed. Both of these errors will be treated as random errors in this discussion; the values for the variance of pressure, temperature, and salinity due to measurement error are taken from Fruchaud (1975) (see Table 2.2). As estimates of the vertical finestructure errors, values for the variance of the regression estimates over each regression interval $\Delta p$ are calculated from the regression residuals, following Fofonoff and Bryden (1975). An estimate of the variance $\sigma^{2}$ of a single data point, for either temperature or pressure is:

$$
\begin{aligned}
& \sigma_{p}^{2}=\frac{I}{Q-N} \sum_{\nu=1}^{Q}\left(p_{v}-\hat{p}_{v}\right)^{2} \\
& \sigma_{\theta}^{2}=\frac{1}{Q-N} \sum_{v=1}^{Q}\left(\theta_{v}-\hat{\theta}_{v}\right)^{2}
\end{aligned}
$$

with $Q$ the number of observations in $\Delta p, p_{\nu}, \theta_{v}$ the observed pressure and potential temperature, and $\hat{p}_{v}, \hat{\theta}_{v}$ the regression estimates of $p$ and $\theta$ at $\delta_{v}$. Further, estimates of the variance of the regression estimates $\hat{p}, \hat{\theta}$ at any value of $\delta$ are given by 


$$
\begin{aligned}
v(p(\delta))=\sigma_{p}^{2} \sum_{i=1}^{N+1} \sum_{j=1}^{N+1} R_{i j}-1\left(\delta-\delta_{k}\right)^{i+j-2} \\
v(\hat{\theta}(\delta))=\sigma_{\theta}^{2} \sum_{i=1}^{N+1} \sum_{j=1}^{N+1} R_{i j}{ }^{-1}\left(\delta-\delta_{k}\right)^{i+j-2} \\
R_{i j}=\sum_{\nu=1}^{2}\left(\delta_{v}-\delta_{k}\right)^{i+j-2} .
\end{aligned}
$$

Given these estimates for measurement and finestructure errors in pressure, temperature and salinity, errors in derived quantities such as steric anomaly and Brunt-Väisälä frequency can be calculated in the same way as in Gregg (1979), using Taylor series expansions in the independent variables $p, T$ and $S$. The errors in the vertical displacements $\pi_{k}$ :

$$
\pi_{k}=p_{i k}-p_{f}
$$

are given by the exrors in $p_{i k}$ since. $p_{f}$ is chosen and therefore has no error associated with it. (The errors in $p_{i k}$ are just the pressure errors.) Thus the errors in each term of the expansion (1.15) of GPE can be estimated as a function of the displacement error, as described in detail in Appendix A.

The reference specific volume surfaces $\delta_{f}$ have small random errors because of the heavy vertical and horizontal averaging which is inherent in the leveling technique. The polynomial regressions of $p$ and $\theta$ against steric anomaly allow an accurate weighting of the vertical average, without impairing vertical resolution. The measurement errors 
in $\delta_{\mathrm{f}}$ are:

$$
\varepsilon_{\delta}^{2}=\left(\frac{\partial \delta}{\partial T}\right)^{2} \cdot \varepsilon_{T}^{2}+\left(\frac{\partial \delta}{\partial \mathrm{p}}\right)^{2} \cdot \varepsilon_{\mathrm{p}}^{2}+\left(\frac{\partial \delta}{\partial S}\right)^{2} \cdot \varepsilon_{S}^{2}
$$

$\varepsilon_{T^{\prime}} \varepsilon_{\mathrm{p}^{\prime}} \varepsilon_{\mathrm{S}}$ the measurement errors in $\mathrm{T}, \mathrm{p}$, and $\mathrm{S}$ respectively. (Recall that measurement errors are taken to be random here.) Random errors in $\theta_{f}$ and $S_{f}$ mapped on $\delta_{f}$ surfaces are also small, since $\theta$ (and implicitly S) are known as functions of $\delta$; hence random errors in $\theta_{f}$ and $s_{f}$ are estimated by the product of the averaged gradients $\left(\partial \theta / \partial \delta_{f^{\prime}} \partial S / \partial \delta_{f}\right)$ and the small random errors in $\delta_{f^{*}}$. These two types: of averaging (for $\delta_{f}$ and for $\theta_{f}$ separately) combine to reduce significantly the random errors in $\theta$ and $S$ mapped on reference steric surfaces $\delta_{\mathrm{f}^{-}}$.

As discussed by Gregg (1979) other less heavily averaged techniques for mapping $\theta$ and $S$ on density surfaces can introduce large random errors due to finestructure. Uncorrected bias exrors resulting from the use of more than one CTD can also introduce large exrors. The latter are reduced by the adiabatic leveling technique, since $\theta$ and $\mathbf{S}$ are computed as functions of $\delta$, rather than $p$, and the averaged gradients of $\theta$ and $s$ with $\delta$ are less variable than the local gradients with $p$. For example, if the standard method outlined by Gregg is used to map $\theta$ and $S$, then the bias error in potential temperature can be estimated as

$$
\Delta \theta \stackrel{\cong}{=} \Delta_{\delta}\left(\frac{\partial \theta}{\partial p}\right)_{\text {local }} /\left(\frac{\partial \delta}{\partial p}\right)_{\text {Iocal }}
$$

whereas the estimated bias error in $\theta_{f}$ is 


$$
\Delta \theta_{\mathrm{f}} \stackrel{\cong}{=} \Delta_{\delta}\left(\frac{\partial \theta}{\partial \delta_{\mathrm{f}}}\right)
$$

$\left(\Delta_{\delta}\right.$ the bias error in specific volume). Gregg points out that it is primarily the local gradients of $\theta, s$, and $\delta$ with pressure which cause the errors in $\theta$ and $s$ to be large when mapped on density surfaces; that problem does not exist in the determination of $\theta_{f}$ and $s_{f}$. Furthermore, the regressions of $\theta$ and $p$ provide accurate estimates of the vertical finestructure errors in the observed field. The determination of horizontal finestructure errors in $\theta$ and $s$ mapped on reference steric surfaces requires more information than is given by the adiabatic leveling technique alone. In the next section a method is described for determining those errors in a fashion analogous to that given earlier for vertical finestructure.

The errors in derived quantities described above generally vary with depth; typical values are given in Table 2.4 , and more precise estimates are plotted as error bars in the appropriate figures. 
TABLE 2.4

Typical error values for derived variables

Variable

$\pi$

$\delta_{\mathrm{f}}$

$S\left(\delta_{E}\right)$

$\theta\left(\delta_{f}\right)$

$\Delta \mathrm{D}$

GPE

TGPE
Measurement

Error

$\pm 5 \mathrm{ab}$

$\pm 0.5 \times 10^{-5} \mathrm{~cm}^{3} \cdot \mathrm{gm}^{-1}$

$\pm .010 \mathrm{ppt}$

$\pm .010^{\circ} \mathrm{C}$

\pm .1 dyn $\mathrm{cm}$

$\pm 15, \times 10^{-4} \mathrm{~J} \cdot \mathrm{kg}^{-1}$

$\pm 0.02 \times 10^{-4} \mathrm{j} \cdot \mathrm{m}^{-2}$
Vertical Finestructure Error

$\pm 5 \mathrm{db}$

$\pm 0.2 \times 10^{-5} \mathrm{~cm}^{3} \cdot \mathrm{gm}^{-1}$

$\pm .010 \mathrm{ppt}$

$\pm .010^{\circ} \mathrm{C}$

\pm .05 dyn $\mathrm{cm}$

$\pm 10 . \times 10^{-4} \mathrm{~J} \cdot \mathrm{kg}^{-1}$

$\pm .01 \times 10^{-4} \mathrm{~J} \cdot \mathrm{m}^{-2}$ 


\subsection{Mean fields and non-seasonal variability}

As a background to the discussion of seasonal variability, a description of the time mean fields in the region $2^{\circ}$ to $12^{\circ} \mathrm{W}, 43^{\circ}$ to $48^{\circ} \mathrm{N}$ is presented here. The discussion is divided into three parts: the first deals with variables which provide a basic description of the physical system (reference steric field, buoyancy frequency, potential temperature-salinity relationship); the second with variables related to the energetics of the system (vertical displacements, dynamic height, $A P E)$; and the third with variables which may be used as tracers (salinity anomaly, temperature variance).

The vertical structure of the reference steric field $\delta_{f}$ changes very lit.le with time. A typical plot of $\delta_{f}$ vs. pressure is shown in Fig. 2.2. There is a deep nearly mixed layer extending to about $400 \mathrm{~m}$, with a weak main pycnocline below, and very little change in $\delta_{f}$ below the pycnocline. The average buoyancy frequency (N) profile in Fig. 2.3, which was constructed by averaging $\mathrm{N}$ horizontally at each pressure $p_{f}$ for each cruise, and then averaging cruises, shows the same structure in a different perspective. There is a seasonal thermocline which is not plotted. (The adiabatic leveling technique is not valid shallower than about $300 \mathrm{~m}$, since densj.ty surfaces are not continuous horizontally, but may intersect the surface of the ocean. All levels shallower than $300 \mathrm{~m}$ have thus been excluded from consideration here.) The average $\theta$ vs. S relationship is plotted in Fig. 2.4, along with the worthington and Metcalf (1961) $\theta$ vs. S standard curve for the western North Atlantic for comparison. The dominance of Med water influence below the main thermocline is clear. 
Figure 2.2 Reference specific volume anomaly $\delta_{f}(p)$ for Phygas 42. 
PHYGAS 42 REFERENCE SPECIFIC VOLUME ANOMALY, $\delta_{f}\left(10^{5} \mathrm{~cm}^{3} \cdot \mathrm{gm}^{-1}\right)$

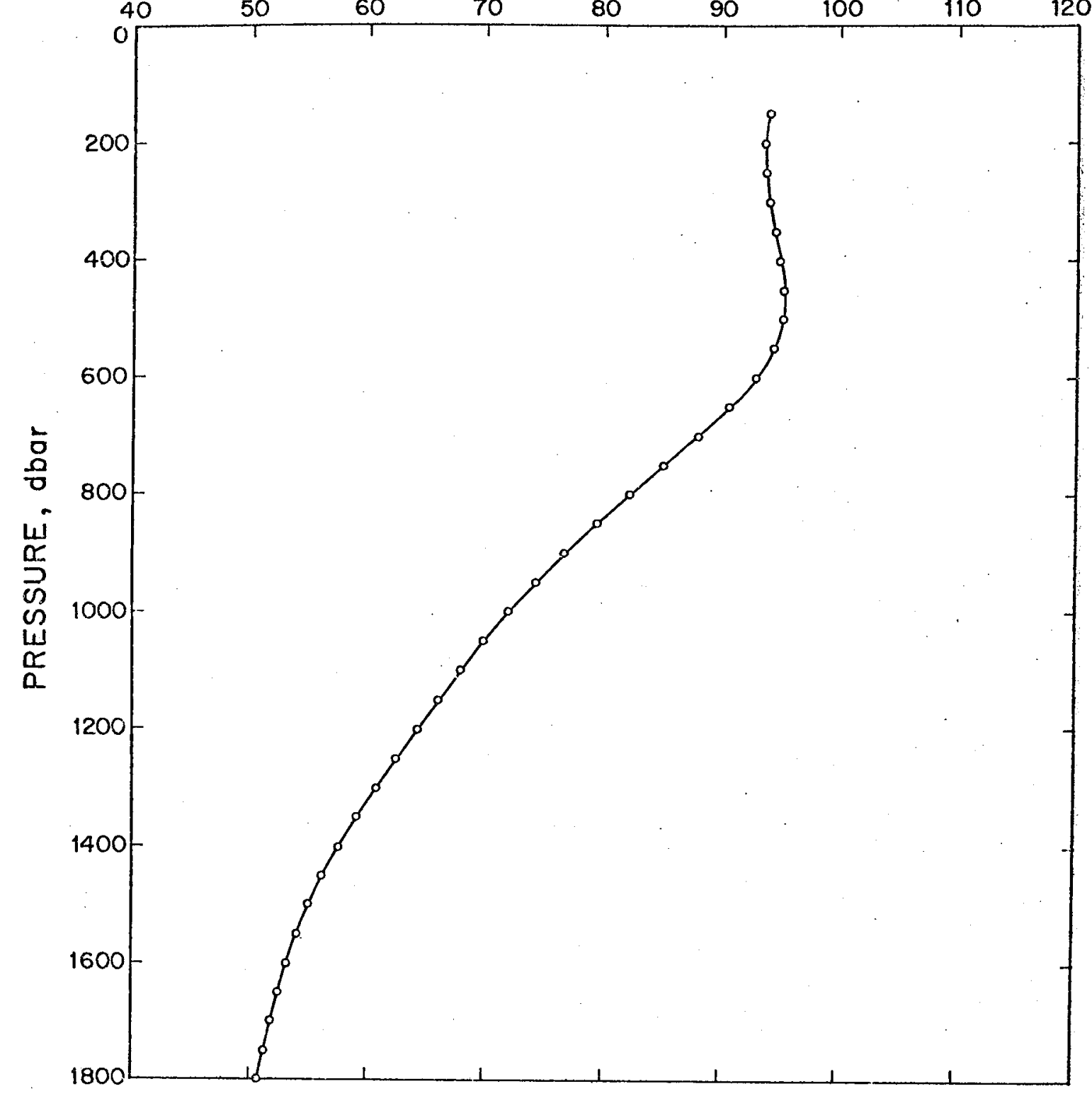


Figure 2.3 Buoyancy frequency N, averaged over all stations, with the range of values from individual cruise averages given by horizontal lines at each depth. 


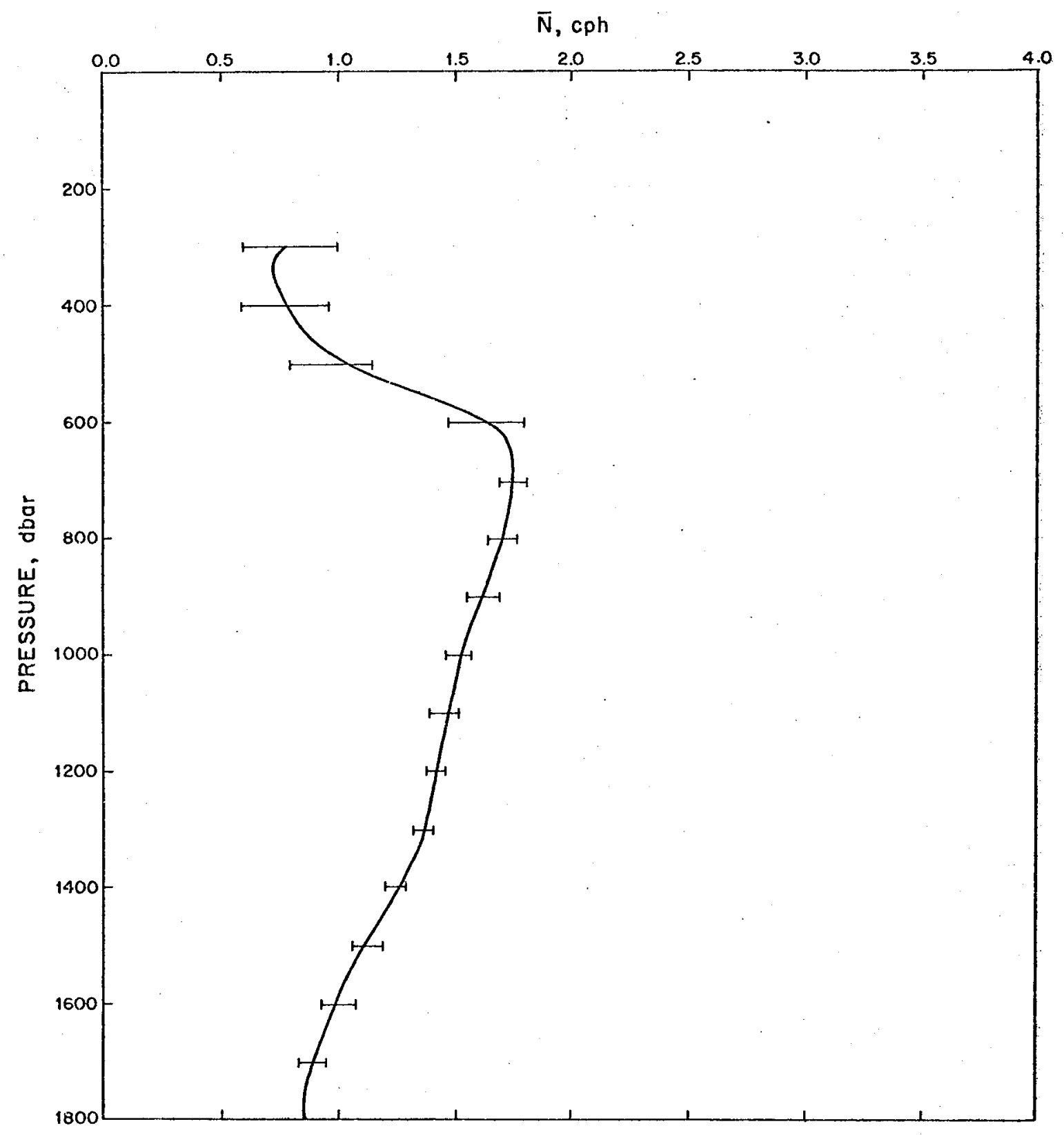


Figure 2.4 Average $\theta$ vs $s$ for all cruises (solid line). Dotted line is a cubic spline fit to $\theta$ vs $S$ from Worthington and Metcalf (1961) and Iselin (1939) for the western North Atlantic. 


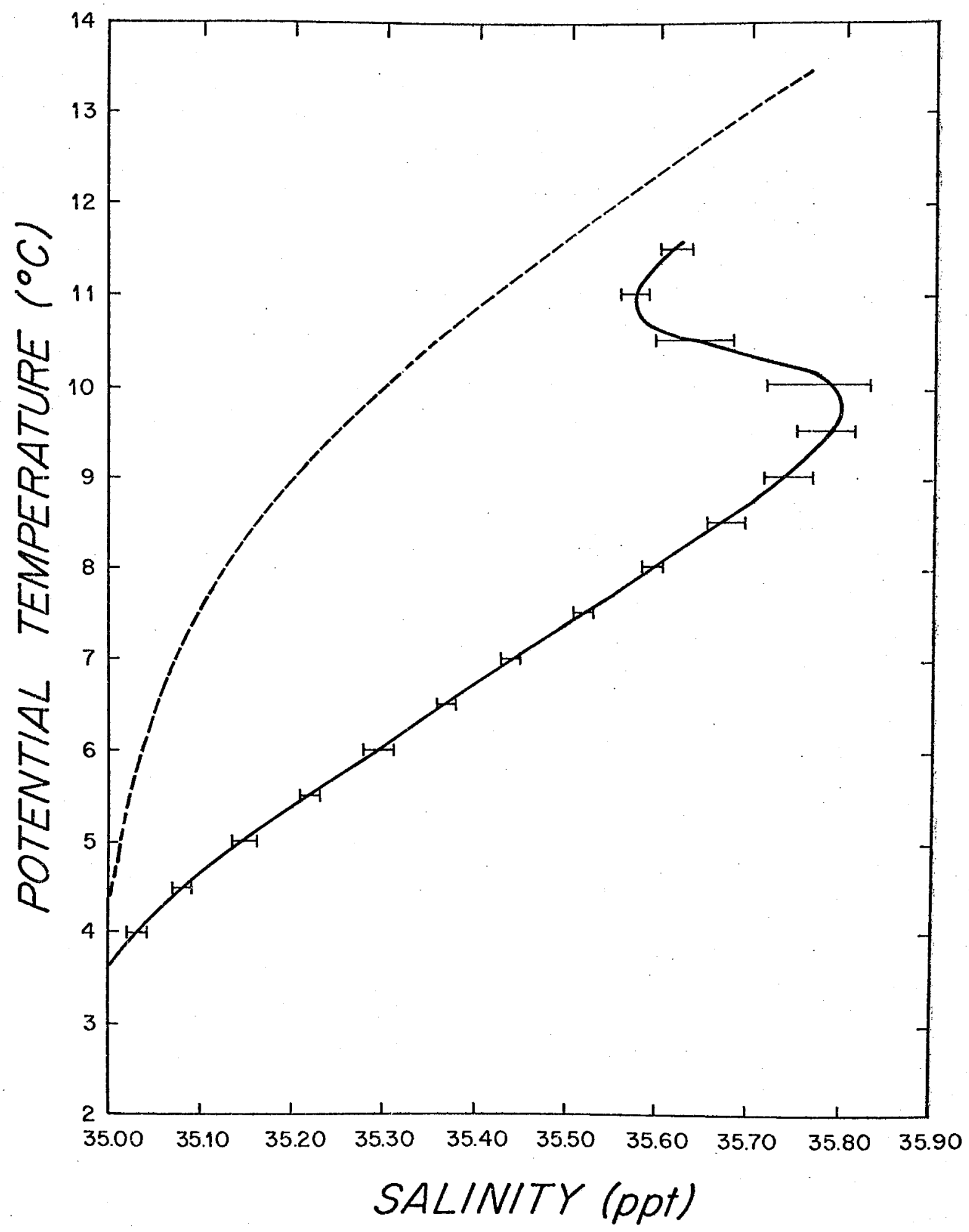


The root mean square (rms) vertical displacements for each pressure $\mathrm{p}_{\mathrm{f}}$ and cruise are shown in Fig. 2.5. Neither the average stratification nor the rms displacements bears a strong resemblance to the typical plot of GPE shown in Fig. 2.6, which has a strong second maximum below the main thermocline. However, a plot of GPE with vertical structure similar to Fig. 2.6 can be constructed by multiplying the average $\mathrm{N}^{2}$ and $\zeta^{2}$ (displacement in meters) at each level as shown in Fig. 2.7. This is a Boussinesq type of estimate, except that correlations between $\zeta$ and $\mathrm{N}$ are neglected, so that the amplitude which results differs from that of a typical plot of GPE. It is the combination of a slowly decreasing stratification and increasing rms displacement which causes the second maximum in GPE below the thermociline. For a comparison with western North Atlantic GPE estimates the reader is referred to Bray and Fofonoff (1980) who discuss the application of the adiabatic leveling technique to the Mid-ocean Dynamics Experiment (MODE). The second maximum in GPE does not appear in any of the MODE GPE profiles. Estimates of eddy kinetic energy (KE) from a current meter mooring located at $47^{\circ} \mathrm{N}, 10^{\circ} \mathrm{W}$ as part of the NEAD observational program are also plotted in Fig. 2.6 (Colin de vediere, personal communication). In the thermocline GPE exceeds KE by a factor of 3; because of the second maximum in GPE below the thermocline, GPE at $1500 \mathrm{db}$ exceeds KE there by a factor of 8 , suggesting that there is storage of potential energy in the density field. The vertical integral of GPE (TGPE) between 300 and $1100 \mathrm{db}$ has a mean value over all cruises of $0.500 \times 10^{-4} \mathrm{~J} \mathrm{~m}^{-2}$ (Table 2.5). Measurement and finestructure errors in TGPE are also given in Table 2.5. 
Figure 2.5 Rms vertical displacements for all cxuises. 


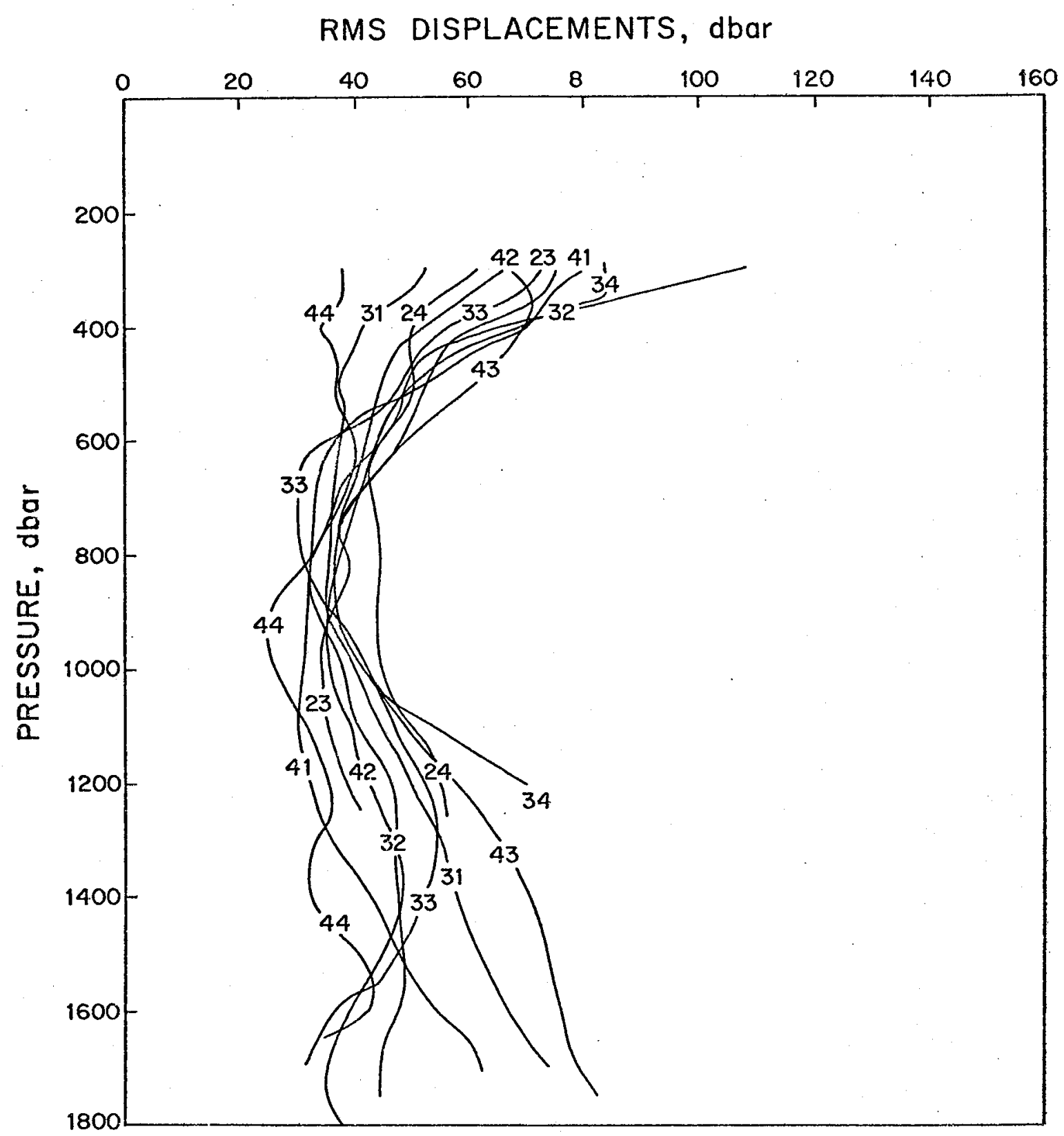


Figure 2.6 APE for Phygas 42. Open circles are GPE, squares are $\mathrm{APE}_{\mathrm{B}^{\prime}} \mathrm{x}$ and + are the contributions from horizontal and vertical gradients of compressibility respectively, and triangles are the sum of the Taylor expansion (1.15). Error bars with heavy end lines represent measurement errors, those with lighter lines represent finestructure errors. Isolated solid circles at 600,1000 , and $1500 \mathrm{db}$ are estimates of eddy kinetic energy (tides and inertial motions removed) from a current meter mooring at $47^{\circ} \mathrm{N}, 10^{\circ} \mathrm{W}$ designated NEAD7. 


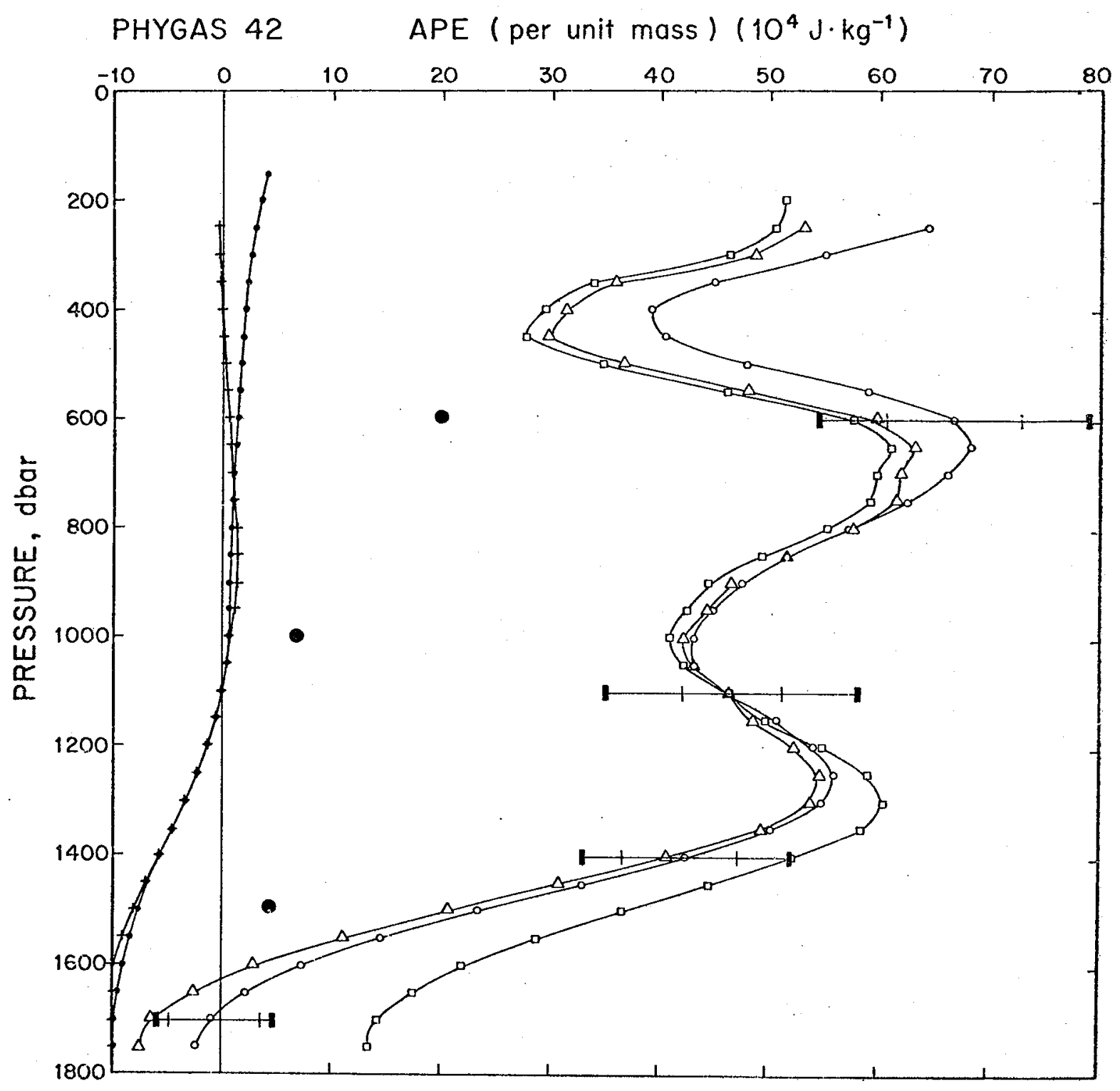


Figure 2.7 Comparison of vertical structure of $.5 \cdot \overline{\mathrm{N}}^{2} \cdot \bar{\zeta}^{2}$ (solid line) and $\mathrm{APE}_{\mathrm{B}}$ for Phygas 42 (dashed line). 


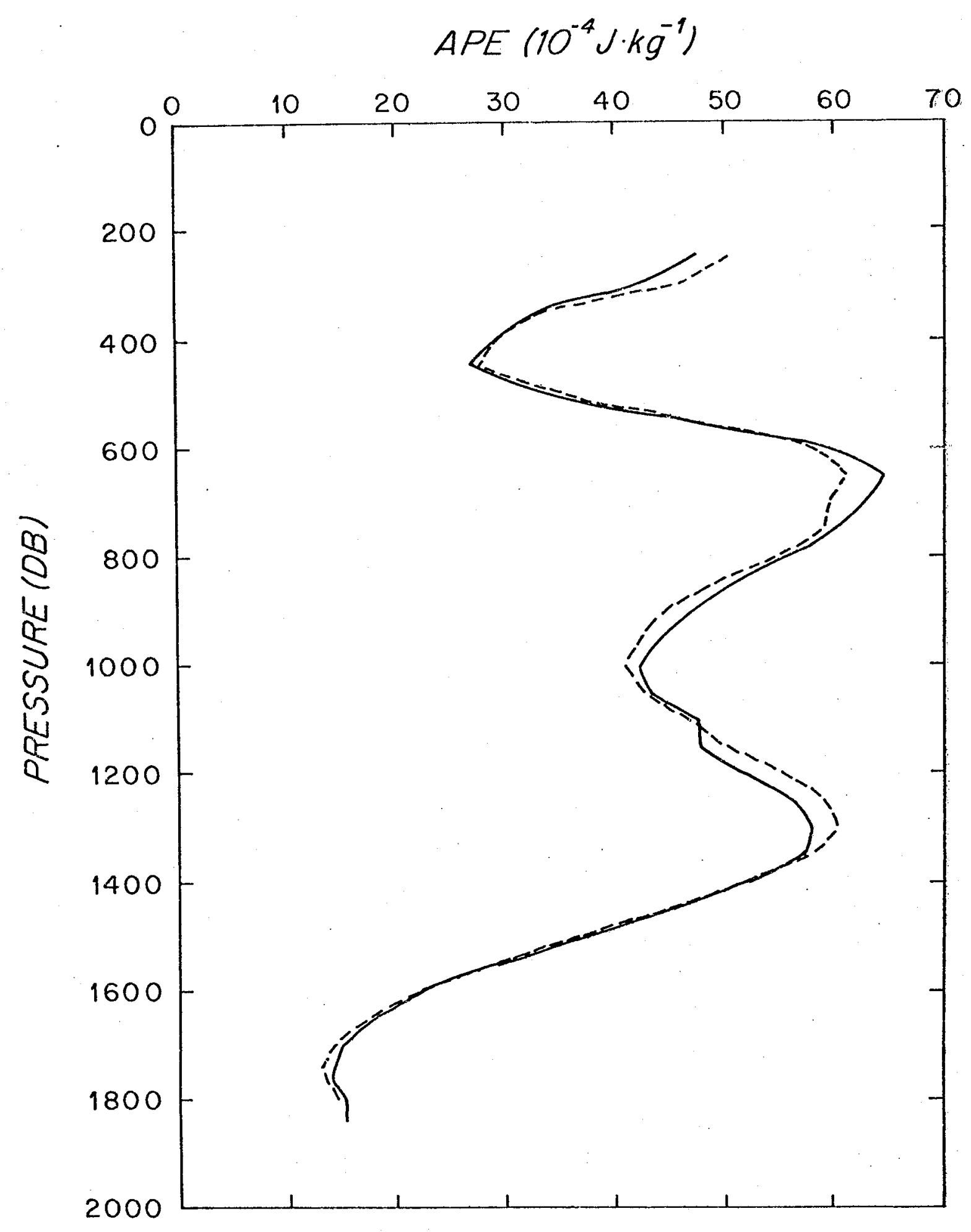


TABLE 2.5

Potential Enexgy Per Unit Area

Cruise

TGPE

$\triangle \mathrm{P} . \mathrm{E}$.

Errors ()

Cruise

$$
10^{-4} \mathrm{~J} \cdot \mathrm{m}^{-2} \quad 10^{-4} \mathrm{~J} \cdot \mathrm{m}^{-2}
$$

22

23

24

31

32

33

34

41

42

43

44
1.043

0.465

0.609

0.416

0.485

0.384

0.525

0.342

0.409

0.559

0.261

Mean (std dev): $\quad .500(.206) \quad-0.36(4.99)$

14.0

2.2

1.3

2.2

3.1

3.1

1.5

0.48

1.22

0.36

$-2.58$

5.2

1.5

1.6

10.4

4.7

3.9

4.8

4.5

5.2

4.0

5.2

4.9

4.1

2.3

6.9

$1 \quad \Delta \mathrm{P} . \mathrm{E} .=\frac{1}{\mathrm{~g}} \int_{1100}^{300} \int_{1100}^{\mathrm{P}}\left[\delta_{f}(n+1)-\delta_{f}(n)\right] d p^{\prime} d p, n$ the sequential

number of the cruise. 
Dynamic height at $500 \mathrm{db}$ referxed to $1000 \mathrm{db}$ calculated according to (1.25) is plotted for each cruise in Figs. 2.8 to 2.10 . The plots are objective maps with an imposed correlation scale of $100 \mathrm{~km}$, as indicated by correlation function calculations. The signal in dynamic height is weak (a maximum of \pm 3 dyn $\mathrm{cm}$ ) and generally correlated with the dispacements $\pi$ between 500 and $1000 \mathrm{db}$ (also plotted in the dynamic height maps). The dynamic height field is not steady but shows evidence of slow westward propagation of features at 2 to $5 \mathrm{~cm} \mathrm{sec}^{-1}$ (Fig. 2.11)

Because the changes in salinity over the field are small comparea to the salinity itself, and features of interest may be obscured, salinity anomaly (defined as the difference between the observed salinity and the salinity which a parcel of water at the observed potential temperature would have if it adhered to the Worthington-Metcalf and Iselin $\theta-s$ curves) was examined. Armi (personal communication) fitted a cubic spline to the combined Worthington and Metcalf (1961) $\theta-5$ curve for the western North Atlantic and a modified version of Iselin's (1939) T-S curve for the intermediate and warmer waters, in which temperature was converted to potential temperature using standard temperature-pressure correlations from hydrographic data. The coefficients for this cubic spline fit, which were modified slightly in the near surface waters by Maillard (personal communication) are found in Appendix B. The curve is plotted in Fig. 2.4.

The average over all cruises of the horizontally averaged salinity anomaly on reference steric surfaces is plotted in Fig. 2.12. There is some change in the average structure between cruises; however, the horizontal average for a given cruise is biased by the location of the stations 
Figures 2.8 objective maps of dynamic height at 500 relative to $1000 \mathrm{db}$ to 2.10 for all cruises (contour units are 1 dyn. cm.). The horizontal coordinates are kilometers from the origin position, chosen to be $45.5^{\circ} \mathrm{N}, 7^{\circ} \mathrm{W}$. Superimposed upon the dynamic height contours are the displacements $\pi$ as a function of depth for each station. The symbol identifies the cruise and is located at the geographical position of the station. It also marks the $1000 \mathrm{db}$ depth on the pressure axis. The scales for pressure and displacement are given in the inset on each figure. The cruises are divided up by year: Fig. 2.8 is 1972 (Phygas 22, 23,24), Fig 2.9 is 1973 (Phygas 31, 32, 33, 34) and Fig. 2.10 is 1974 (Phygas $41,42,43,44$ ). 


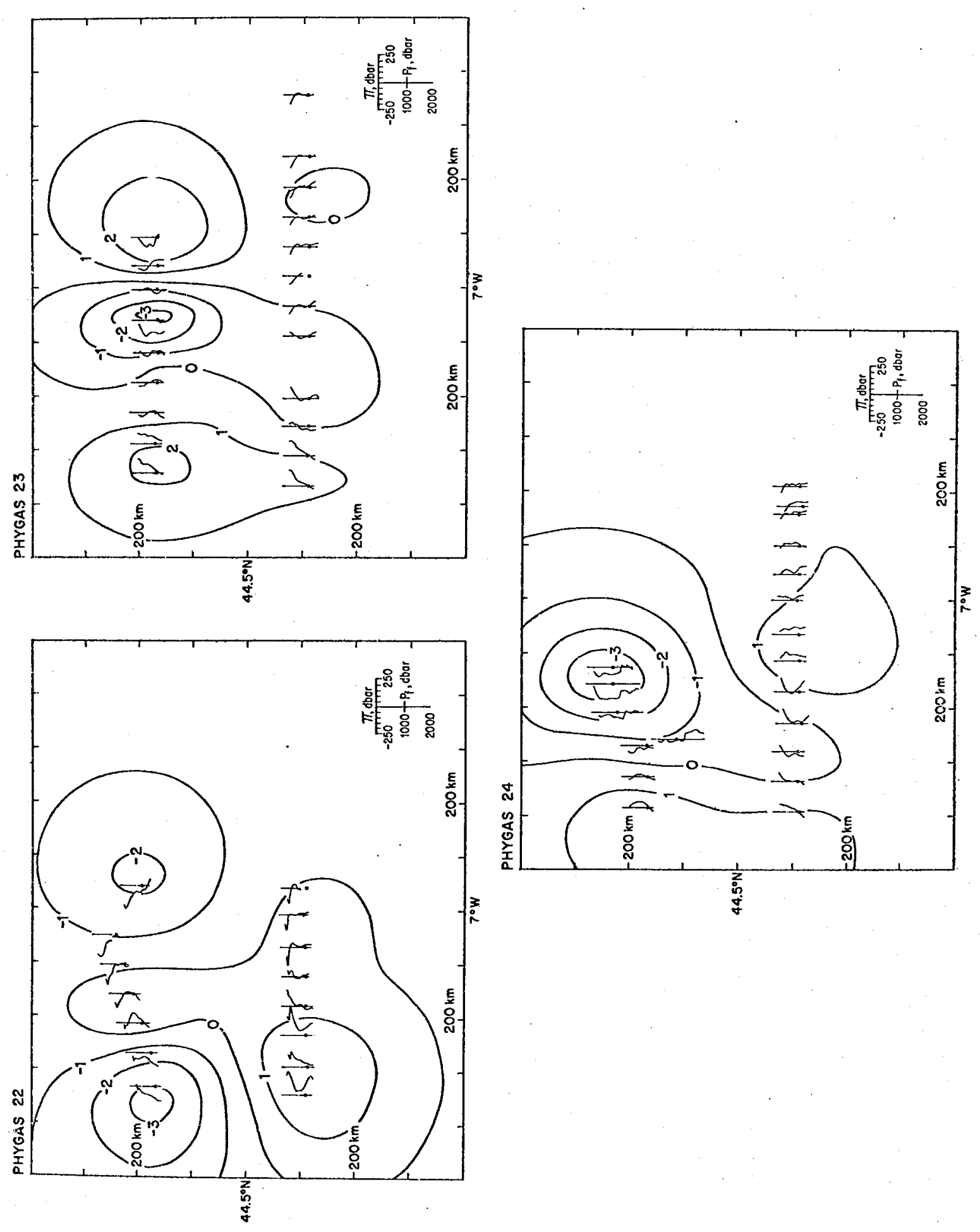



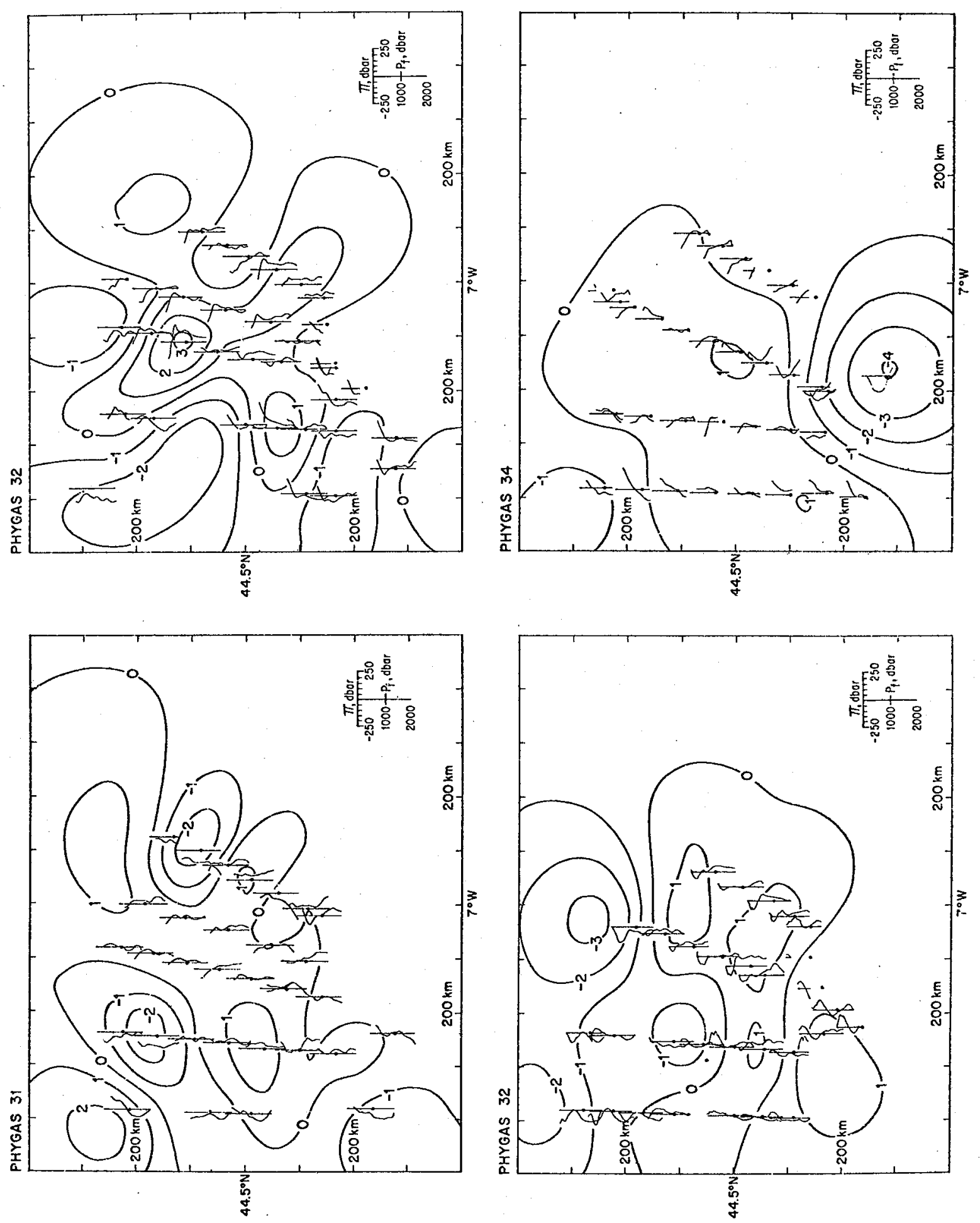

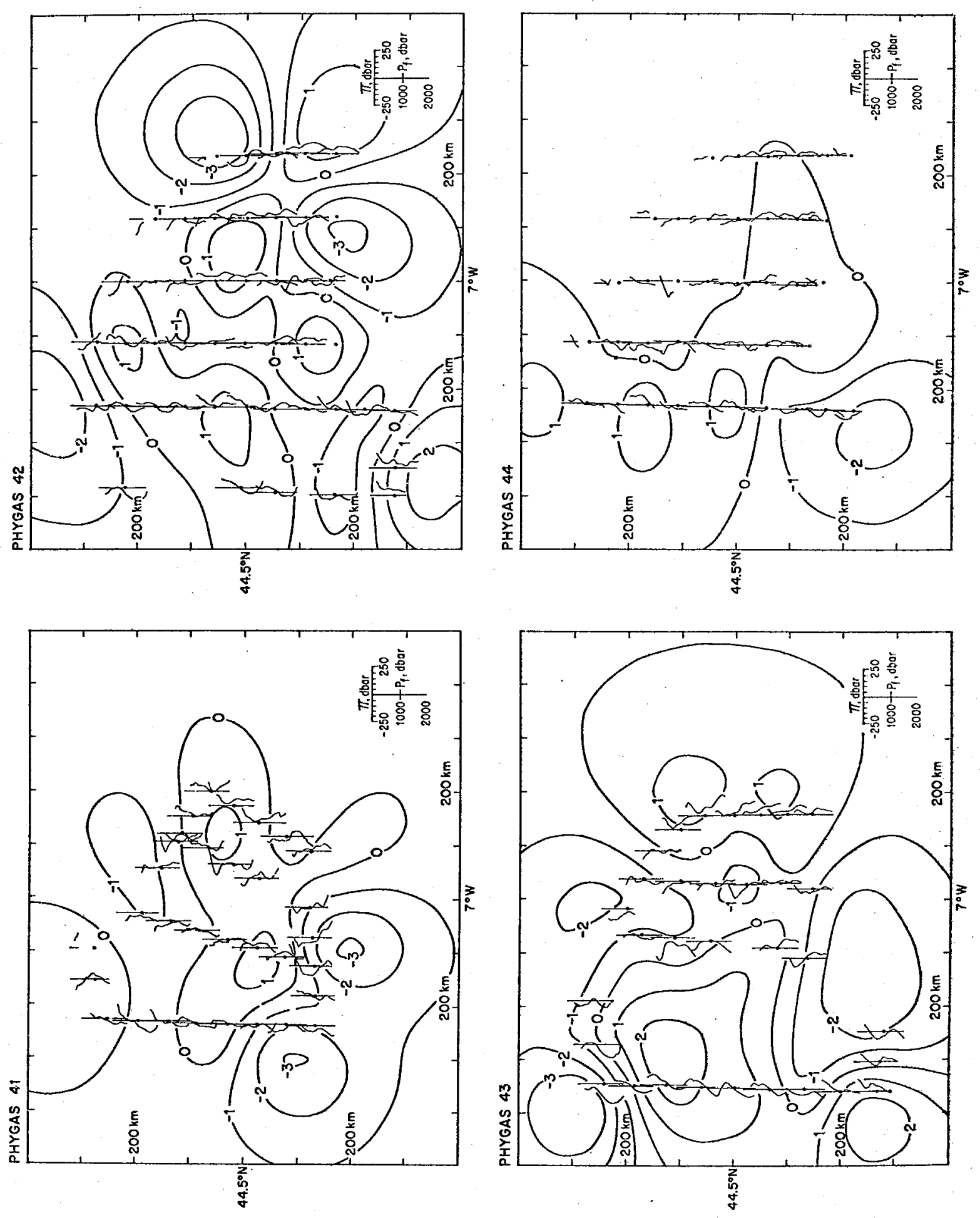
Figure 2.11 Contour plot of dynamic height as a function of time (vertical axis) along a line $200 \mathrm{~km}$ south of the origin $\left(45.5^{\circ} \mathrm{N}, 7^{\circ} \mathrm{W}\right)$. Contour interval is 1 dyn.cm. 


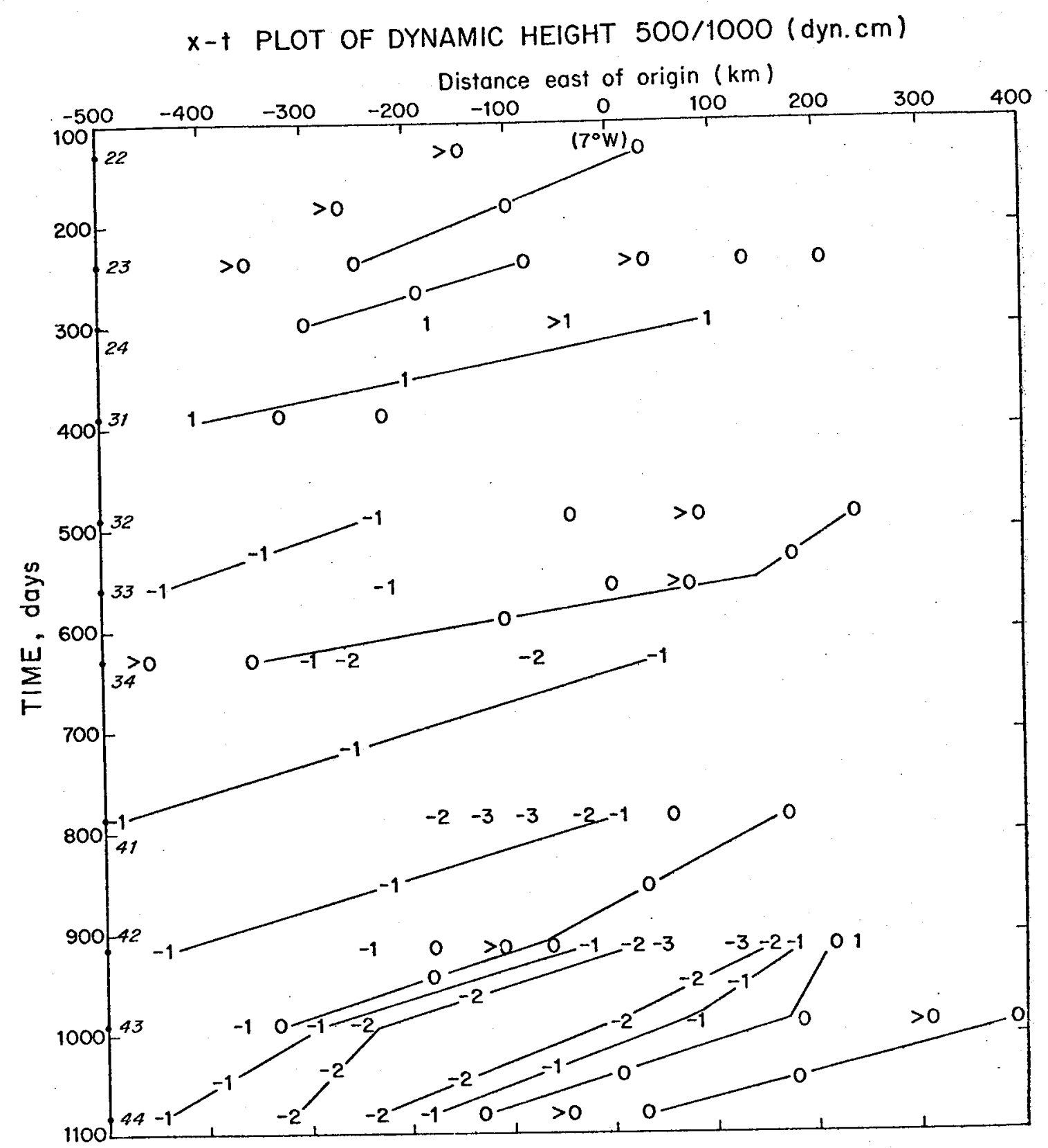


Figure 2.12 Salinity anomaly vs pressure: average over all cruises. Horizontal lines indicate range of values from individual cruise averages. 


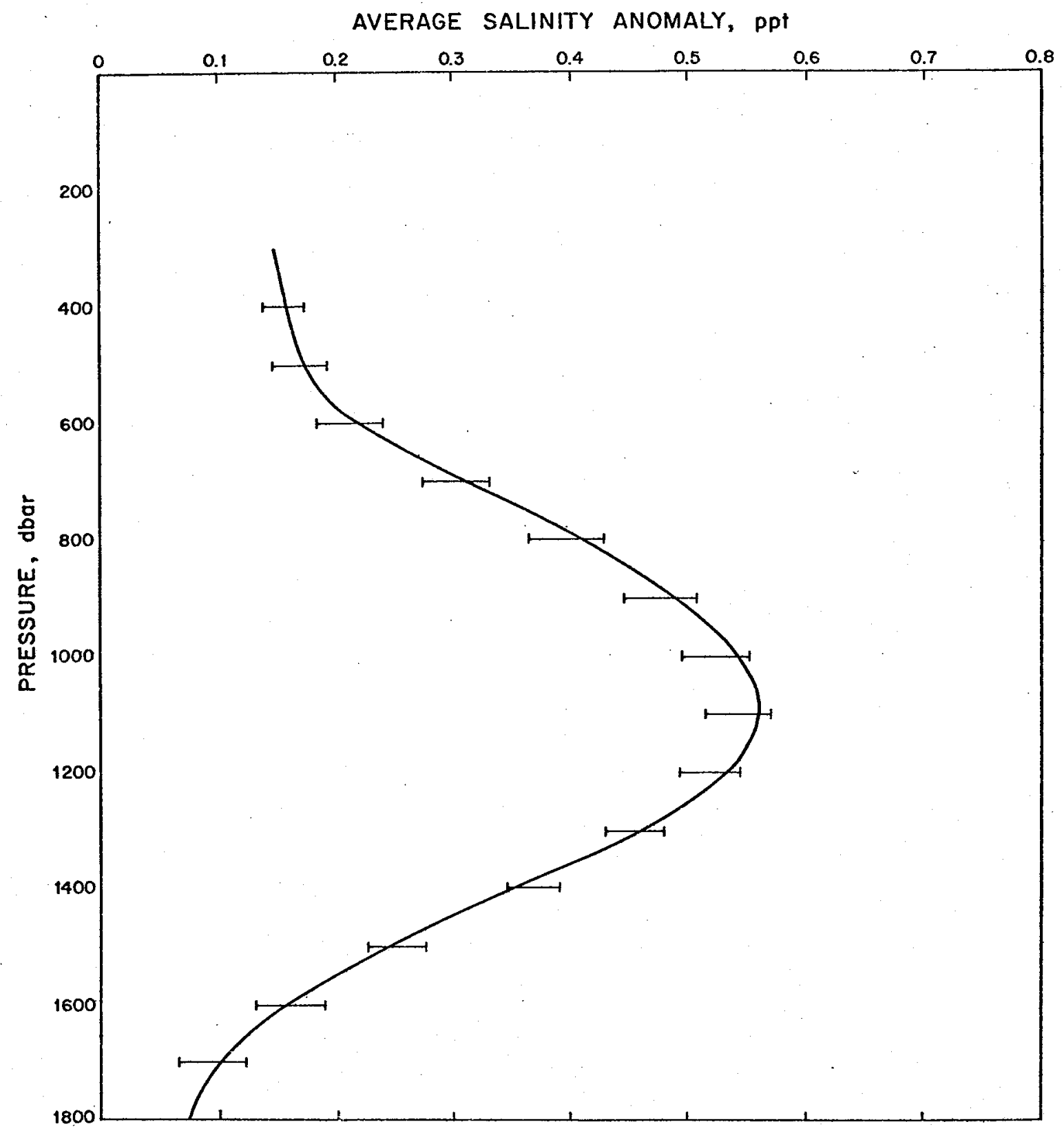


since there are strong mean horizontal gradients, as can be seen in the objective maps of salinity anomaly on the reference surface at 1000 dbar (Figs. 2.13-2.15).

The mean horizontal structure of the salinity anomaly field was determined using a least squares linear regression in $x$ (east) and $y$ (north) distance from the origin at $45.5^{\circ} \mathrm{N}, 7^{\circ} \mathrm{W}$ (see Fig. 2.1):

$$
s=\sum_{j=0}^{M} \sum_{i=0}^{N} s_{i j}\left(x-x_{0}\right)^{i}\left(y-y_{0}\right)^{j}
$$

with $x_{0}$ and $y_{0}$ the origin coordinates. These regressions were performed over steric surfaces correspording to $400,600,800,1000$ and $1200 \mathrm{db}$ for all cruises, and also over 1400, 1600, $1700 \mathrm{db}$ for cruises with sufficiently deep data. The optimum number of terms in the regression was determined by examining the statistical confidence of the ratio of each coefficient $\Delta S_{i j}$ to its standard deviation. Four terms were identified at $95 \%$ confidence for most levels and cruises: $\Delta S_{00}$, the average value, $\Delta \mathrm{S}_{10}$, the zonal $(\mathrm{x})$ gradient: $\Delta \mathrm{S}_{01}$, the meridional (y) gradient; and $\Delta \mathrm{S}_{20}$ ' the zonal curvature or second derivative. The mean zonal and meridional gradients (ụsing all stations) are listed in Table 2.6. Note that the gradients are evaluated at the origin. Horizontal finestructure errors in salinity anomaly were estimated by examining the variance of the regression estimate of $\Delta_{S}$ as was done in section 2 to determine the vertical finestructure errors in $\theta$ and $p$. The estimates of error in $\Delta S$ are plotted in Fig. 2.17. Similar regressions were done using the displacement $\pi$ and the vertical finestructure error $\sigma_{\theta}$. A mean horizontal gradient in $\pi$ would indicate a mean slope in the isopycnals; however, no discernable (i.e., 95\% confidence) grad- 
Figures 2.13 objective maps of salinity anomaly at $1000 \mathrm{db}$ for all to 2.15 cruises. Contour intervals are .050 ppt; imposed correlation scale is $100 \mathrm{~km}$. 


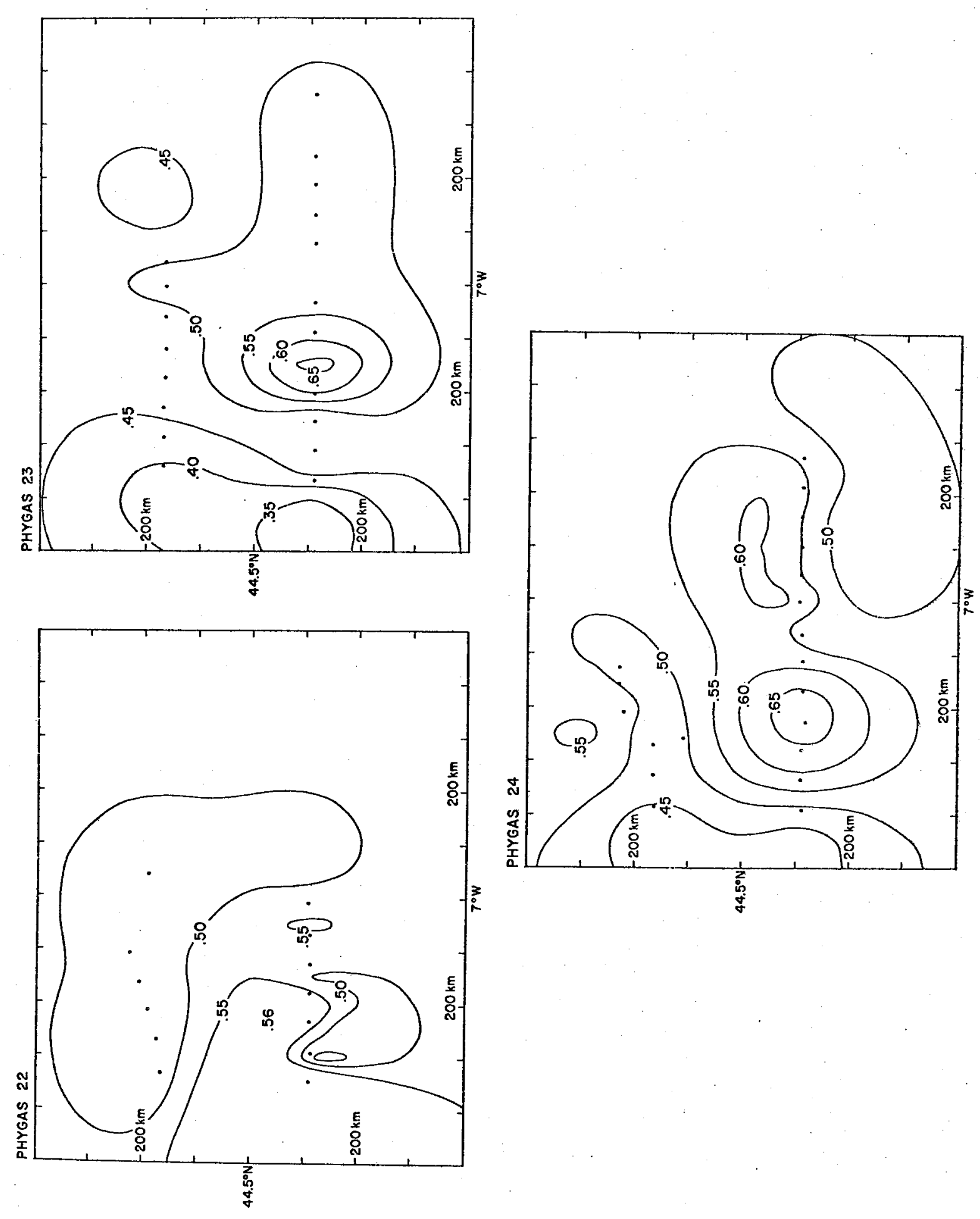



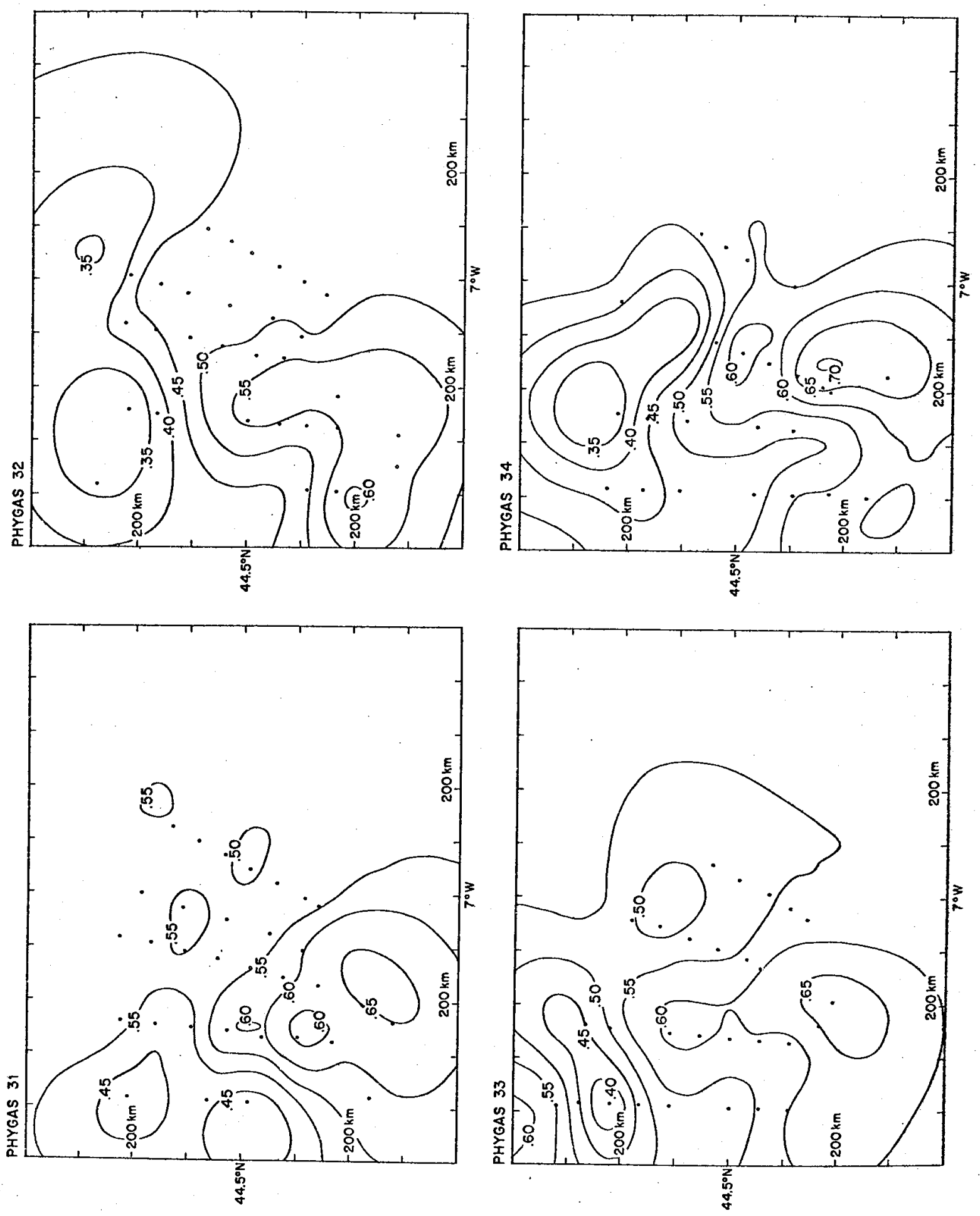

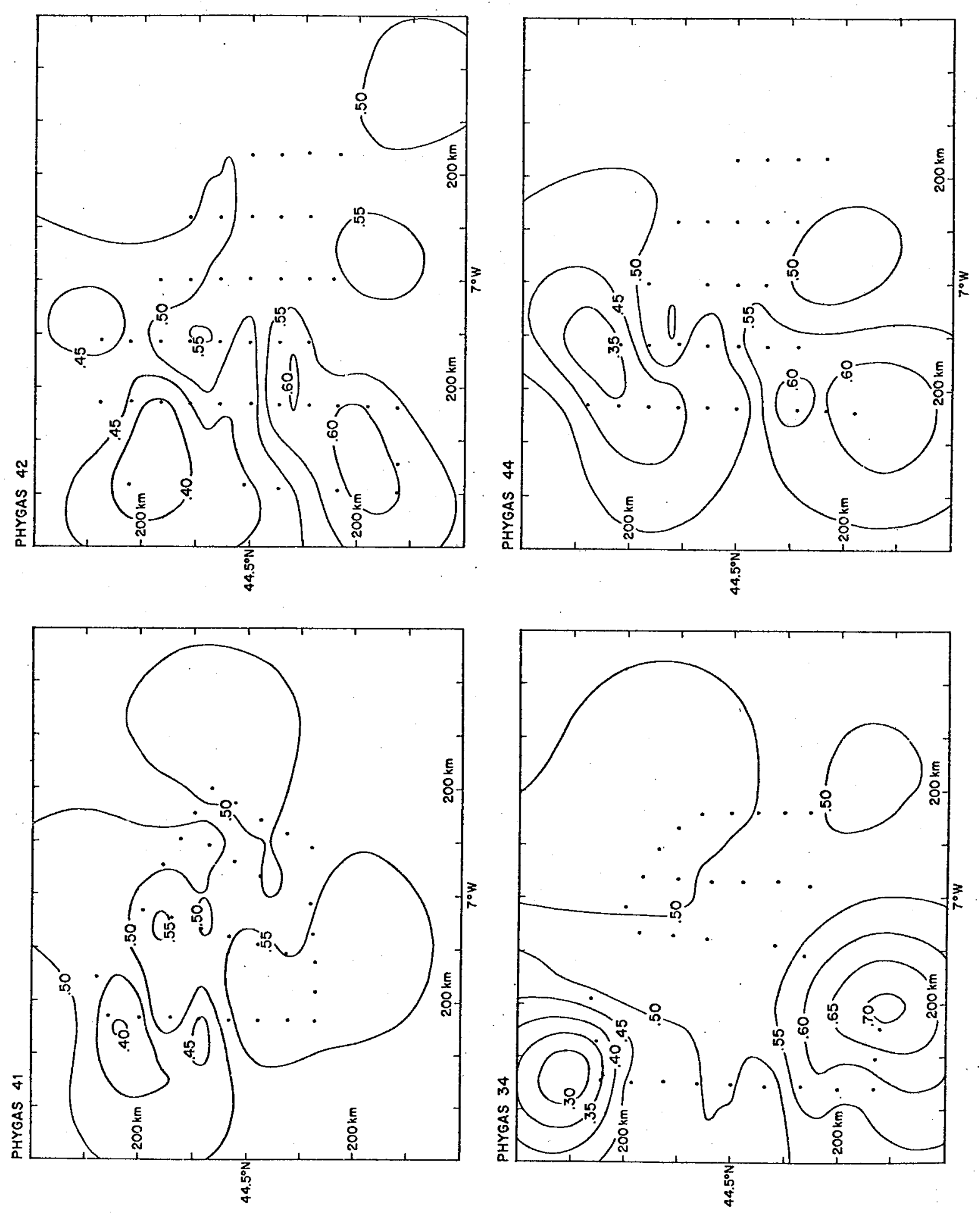
TABLE 2.6

Gradients of Salinity of Anomaly at Origin

\begin{tabular}{|c|c|c|c|c|}
\hline \multirow[b]{3}{*}{ Depth } & Mean ${ }^{3}$ & Mean $^{2}$ & \multicolumn{2}{|c|}{ Number of } \\
\hline & $s_{x}\left(s_{10}\right)$ & $S_{y}\left(s_{0 I}\right)$ & realiza & Lons $^{3}$ \\
\hline & $10^{3} \mathrm{ppt} \cdot \mathrm{km}^{-1}$ & $10^{3} \mathrm{ppt} \cdot \mathrm{km}^{-1}$ & $\mathrm{~s}_{\mathrm{x}}$ & $\mathrm{S}_{\mathrm{y}}$ \\
\hline 400 & $0.00(.03)$ & $-0.05 \quad(.01)$ & 11 (5) & (11) \\
\hline 600 & $-0.02(.04)$ & $-0.09 \quad(.02)$ & 11 (3) & (11) \\
\hline 800 . & $-0.05(.16)$ & $-0.24(.05)$ & 11 (3) & (11) \\
\hline 1000 & $-0.11(.16)$ & $-0.34(.08)$ & 11 (5) & (11) \\
\hline 1200 & $-0.12(.21)$ & $-0.42(.13)$ & 11 (7) & (11) \\
\hline 1400 & $-0.10(.11)$ & $-0.40(.10)$ & $7(1)$ & (7) \\
\hline 1600 & $+0.06(.16)$ & $-0.28 \quad(.03)$ & 7 (1) & (7) \\
\hline 1700 & $+0.08 \quad(.13)$ & $-0.23(.04)$ & $6(1)$ & (6) \\
\hline
\end{tabular}

1 Mean over all cruises of value of $s_{10}$ at origin. In parentheses: standard deviation over all cruises.

2 Mean over all cruises of value of $\mathrm{S}_{\mathrm{Ol}}$ at origin. In parentheses: standard deviation over all cruises.

3 In parentheses: $\dot{S}_{x}=$ - number of realizations for which $s_{10}$ is different from zero at the 95\% confidence level; $S_{y}-$ same as $s_{x}$, except for $\mathrm{s}_{\hat{\mathrm{gl}} \mathrm{I}}$. 
ients in $\pi$ were found. The finestructure error in potential temperature is an indication of the strength of vertical mixing: in general the greater the finestructure, the less well mixed is the fluid. There are strong zonal gradients of temperature finestructure in these data, with the strongest mixing occurring near the boundary (see Table 2.7). The zonal gradient increases with depth between 400 and $1200 \mathrm{db}$ which may simply reflect the strong variability offshore associated with the Med Water which has a maximum at roughly $1200 \mathrm{db}$. The meridional gradient of temperature finestructure is only occasionally discernable at $95 \%$ confidence. 
TABLE 2.7

Zonal Gradient of Temperature Finestructure

$\begin{array}{lll}\text { Mean zonal } & \text { Std. dev. } & \text { Number of } \\ \text { gradient } & & \text { realizations }\end{array}$

$\begin{array}{lccc}\text { Pressure } & \left(10^{-5}{ }^{\circ} \mathrm{C} \mathrm{km}^{-1}\right) & & \\ 400 & 3.9 & 2.7 & 11(8) \\ 600 & 5.3 & 1.7 & 11(9) \\ 800 & 9.6 & 3.7 & 10(8) \\ 1000 & 11.2 & 3.0 & 10(10) \\ 1200 & 13.6 & 3.9 & 10(10) \\ 1400 & 12.0 & 6.0 & 7(4)\end{array}$

I In parentheses: number of realizations significantly different from zero at greater than $95 \%$ confidence. 


\subsection{Seasonal variability}

There are two types of seasonal signal below the main thermocline in the Bay of Biscay data: an oscillation of the reference steric field which necessarily occurs on horizontal scales of at least $1000 \mathrm{~km}$ (the horizontal extent of the data); since $\delta_{f}$ is independent of horizontal. position; and seasonal variations in the horizontal structure of salinity anomaly, which occurs on smaller horizontal scales. Because there are only eleven data points in the time series, a simplified harmonic analysis is used to identify the frequency, amplitude and phase of the signals. Phase and amplitude at a given frequency $\omega$ are calculated by a least squares regression of the form

$$
\begin{aligned}
\psi(t) & =a \cos (\omega t+\phi) \\
& =a_{1} \cos \omega t+a_{2} \sin \omega t
\end{aligned}
$$

with

$$
\begin{aligned}
& a_{1}=a \cos \phi \\
& a_{2}=-a \sin \phi
\end{aligned}
$$

$\psi$ any variable, $\phi$ the phase, and $\cos \omega t$, sin $\omega t$ the independent variables. By stepping through a range of frequency and comparing the statistical confidence of the coefficients $a_{1}$ and $a_{2}$ ' the best fit of frequency can be established. The ratio of each coefficient to its standard deviation for frequencies corresponding to periods of 8 to 16 months are plotted in Fig. 2.16 for salinity anomaly $\left(\Delta \mathrm{s}_{00}\right)$ at $1000 \mathrm{db}$. The only period for which both coefficients have greater than $95 \%$ confidence is 12 months. 
Figure 2.16 Ratios of the coefficients of the harmonic analysis (2.4) to their standard deviations for a range of frequency. The values at frequency $I$ cpy are circled. 

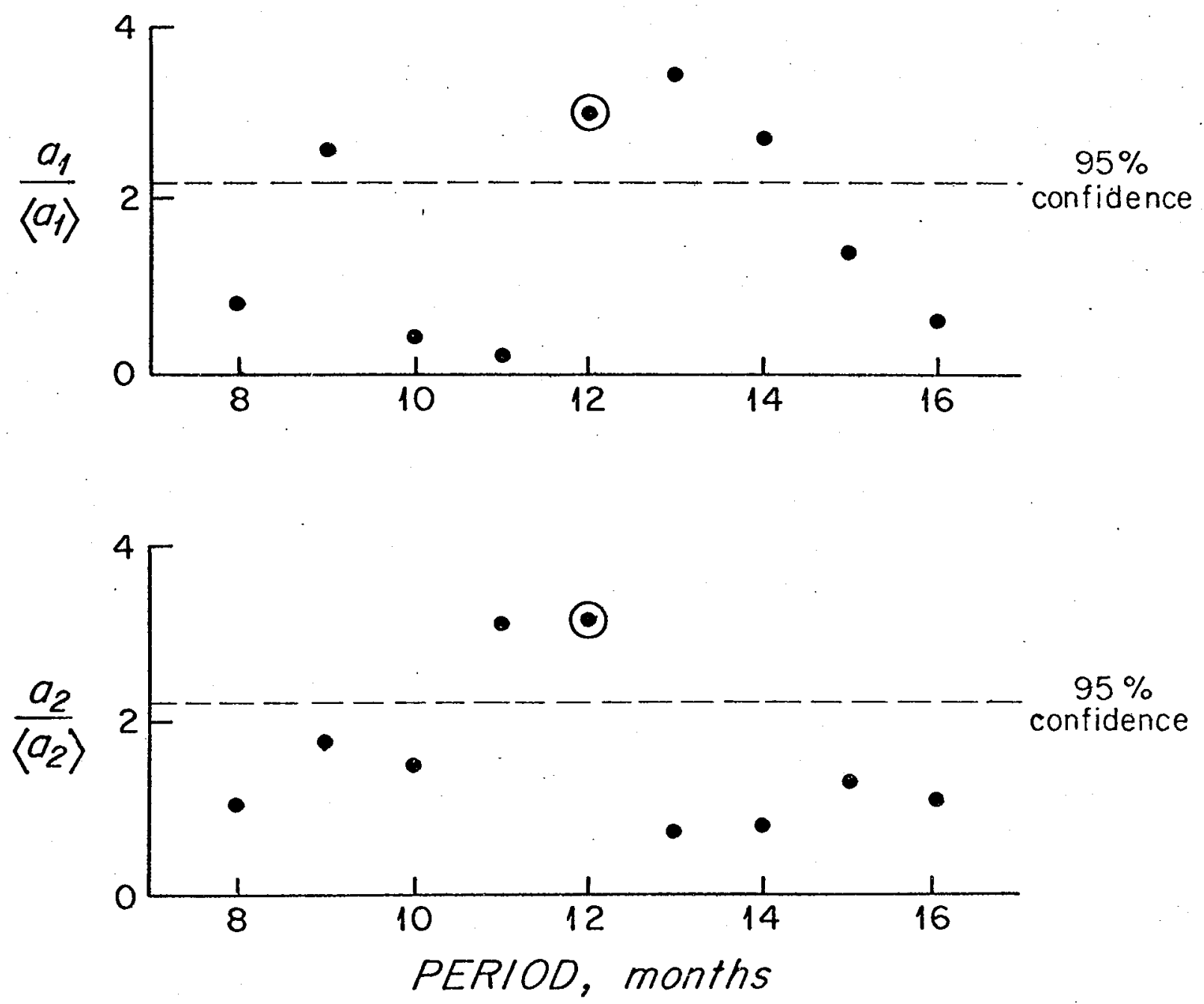
There are seasonal signals also at 800 and $1200 \mathrm{db}$ (Fig. 2.17); there are too few points below 1200 db to identify a seasonal signal with 95\% confidence. The signal double amplitude (peak to trough) is $40 \mathrm{ppm}$ at $800 \mathrm{db}$ and $50 \mathrm{ppm}$ at 1000 and $1200 \mathrm{db}$. For the two deeper levels the phases calculated correspond to maximum values at 130 and 134 days from 1 January. The difference in phase is less than the probable error in phase determination. The signal at $800 \mathrm{db}$ is not strong enough to allow a determination of phase, so a phase of 130 days was used in the regression and only amplitude calculated. None of the higher order terms $\Delta \mathrm{S}_{10}, \Delta \mathrm{S}_{01}, \Delta \mathrm{S}_{20}$ shows a clearly seasonal signal; although $\Delta S_{20}$, the zonal curvature term, does change in time, unlike either gradient term. The change in $\Delta \mathrm{S}_{20}$ cannot be clearly identified as seasonal. A separate analysis of horizontal structure was performed in which the curvature $\left(\Delta s_{20}\right)$ term was omitted from the horizontal regression. The $\Delta S_{\text {oo }}$ terms which result are different from those of the initial calculation and show no seasonal variability. This is interpreted as evidence that the seasonal signal in $\Delta \mathrm{s}_{00}$ results from changes in the horizontal structure of the salinity anomaly field.

The reference steric field also changes seasonally: at 1000 and $1200 \mathrm{db}$ the double amplitude is $2 \times 10^{-5} \mathrm{~cm}^{3} \mathrm{gm}$ with phase set at 130 days from 1 January (see Fig. 2.18). This represents a seasonal displacement of the reference steric surfaces of $\pm 15 \mathrm{~m}$. There is no evidence from this analysis that there is a seasonal signal in the vertical displacements $\pi$; however there are changes in the vertical structure of GPE which may be seasonal (see Fig. 2.19). The strongest maxima (relative to the thermocline) in GPE below the thermocline occur in the winter cruises (31 and 41 ) and summer cruises ( 33 and 43 ). The reference 
Figure 2.17 Salinity anomaly at origin $\left(45.5^{\circ} \mathrm{N}, 7^{\circ} \mathrm{W}\right)$, as estimated by horizontal regression, vs time. Exror bars are horizontal finestructure uncertainties in the coefficient of the zeroth order polynomial term, $\Delta \mathrm{S}_{\mathrm{oo}} \cdot$ Solid lines are harmonic analysis fits of amplitude and phase with frequency 1 cpy. 


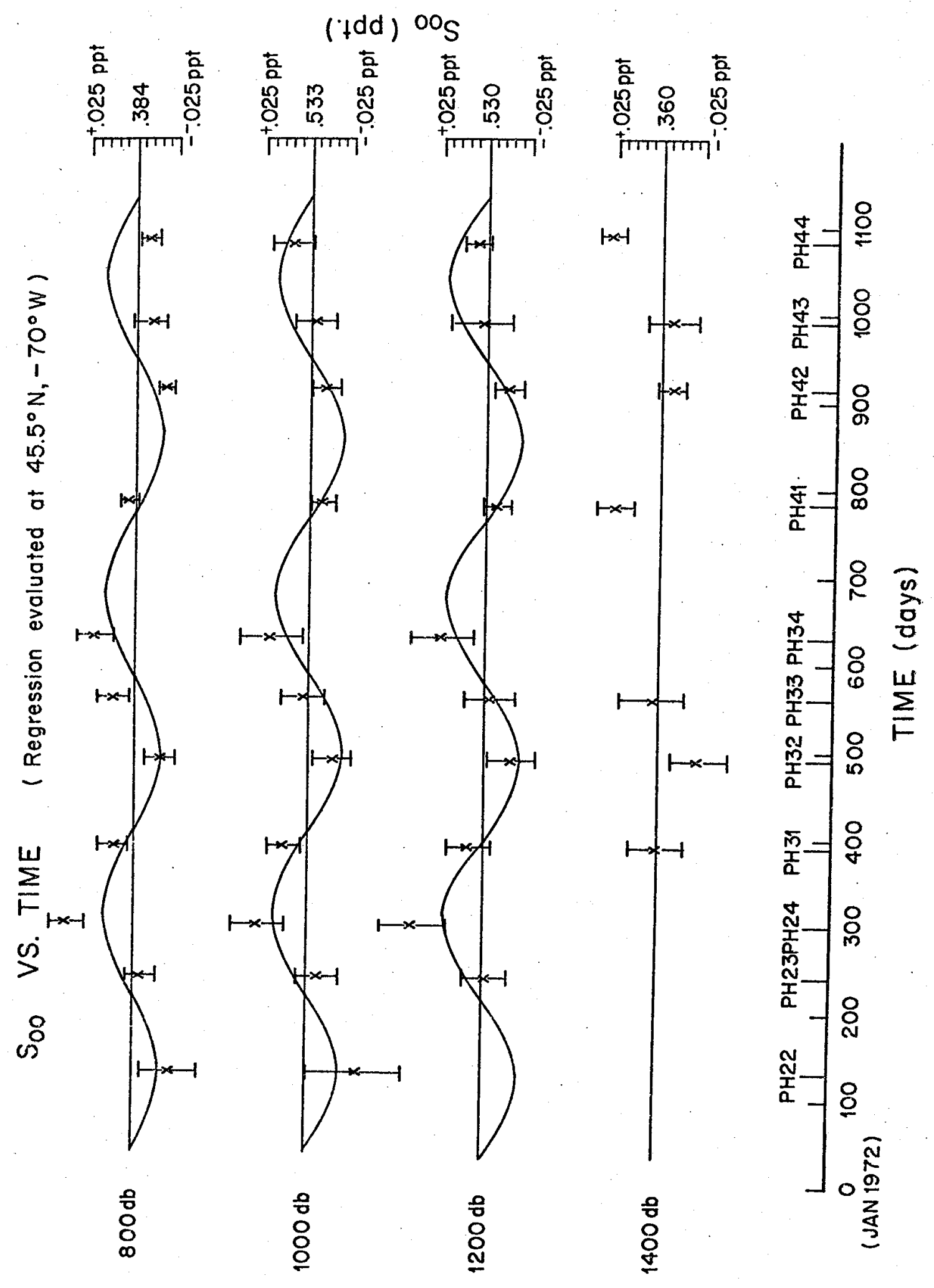


Figure 2.18 Reference specific volume anomaly $\delta_{f}$ vs time. Error bars are measurement errors, which are largex than finestructure errors inferred from differences in uptrace and downtrace calculations. Solid lines are harmonic analysis fits of amplitude; phase is the same as for $1000 \mathrm{db} \Delta \mathrm{s}_{\mathrm{OO}}($ Fig. 2.17) and frequency is $1 \mathrm{cpy}$. 


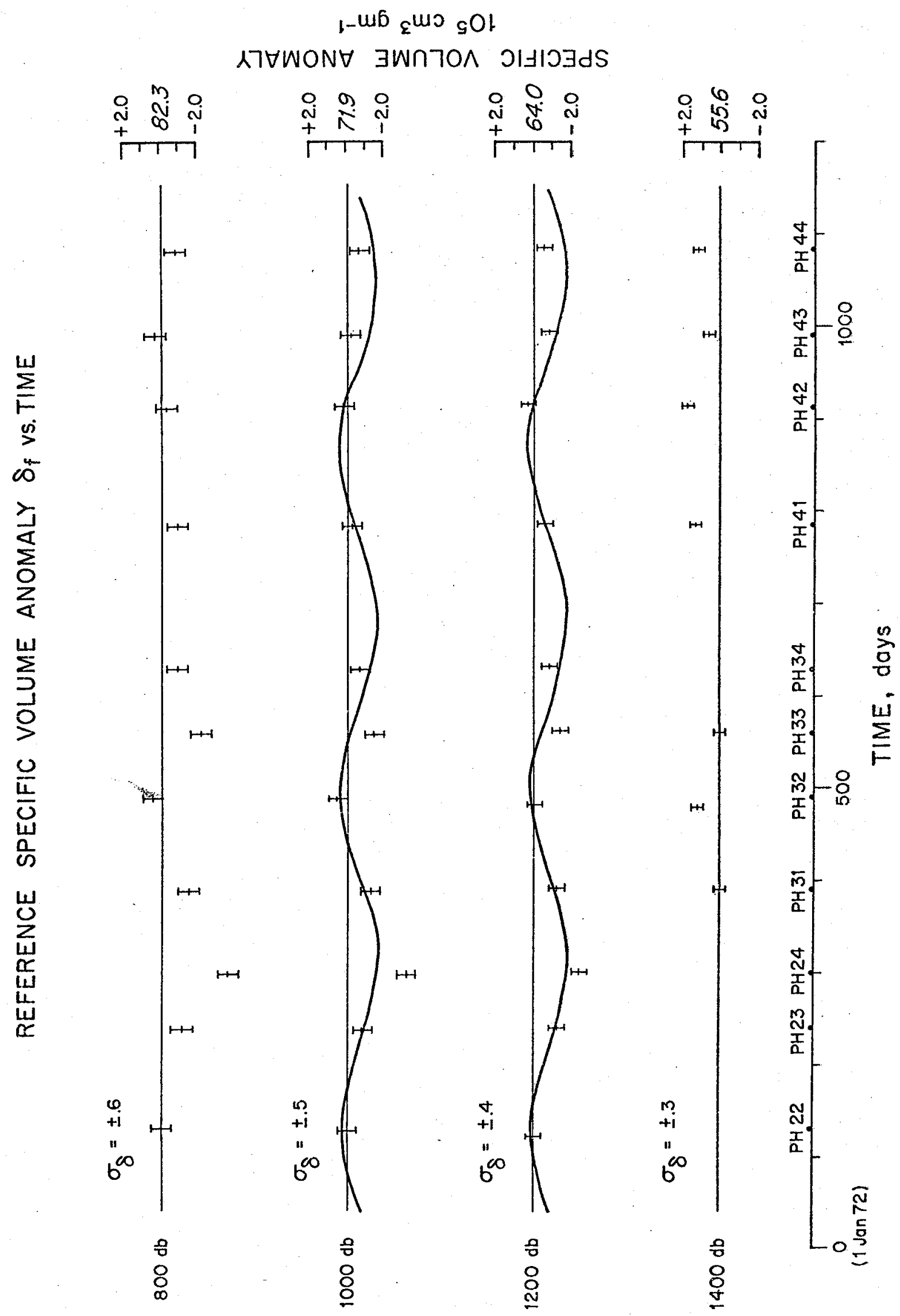


level (1100 ab) GPE (Fig. 2.19, inset) also appears to vary with seasonal periodicity but that signal cannot be identified with $95 \%$ confidence. Of primary interest are the horizontal scales of the different seasonal signals, in particular, that of the salinity anomaly signal. A rough estimate of that scale can be made by comparing the time changes of the zero order term $\left(\Delta \mathrm{S}_{00}\right)$ and the zonal curvature term $\left(\Delta \mathrm{s}_{20}\right)$ :

$$
L^{2} \cong \frac{\frac{\partial}{\partial t} \Delta S_{O O}}{\frac{\partial}{\partial t} \Delta S_{20}} \cong \frac{25 \times 10^{-3} \mathrm{ppt}}{5 \times 10^{-7} \mathrm{ppt} / \mathrm{km}^{2}}
$$

or

$$
\mathrm{L} \cong 200 \mathrm{~km} \text {. }
$$

However, if the signal is actually a!propagating wave, it cannot be identified properly using this technique. Given a zonal wavelength, the data can be fitted to a propagating wave model, with amplitude and phase determined by least squares techniques. The new phase would then contain only time information, not mixed time and space information as it does in this analysis.

In the next chapter a model is constructed in which the wave lengths of the dominant free propagating waves are calculated, and the resulting change in salinity anomaly estimated. The model predicts seasonal variability in the displacement field as well. Using the model prejudice for horizontal scale, the data are refitted to traveling plane waves with seasonal period and good agreement is found between the model and the data in the salinity anomaly signal and also in the displacement field, which is found to have a seasonal signal when the appropriate analysis is applied. 
Figure 2.19 GPE for all cruises, with the reference value (1100 db) subtracted, for comparison of vertical structure. Inset has APE (1100 db) vs time. 


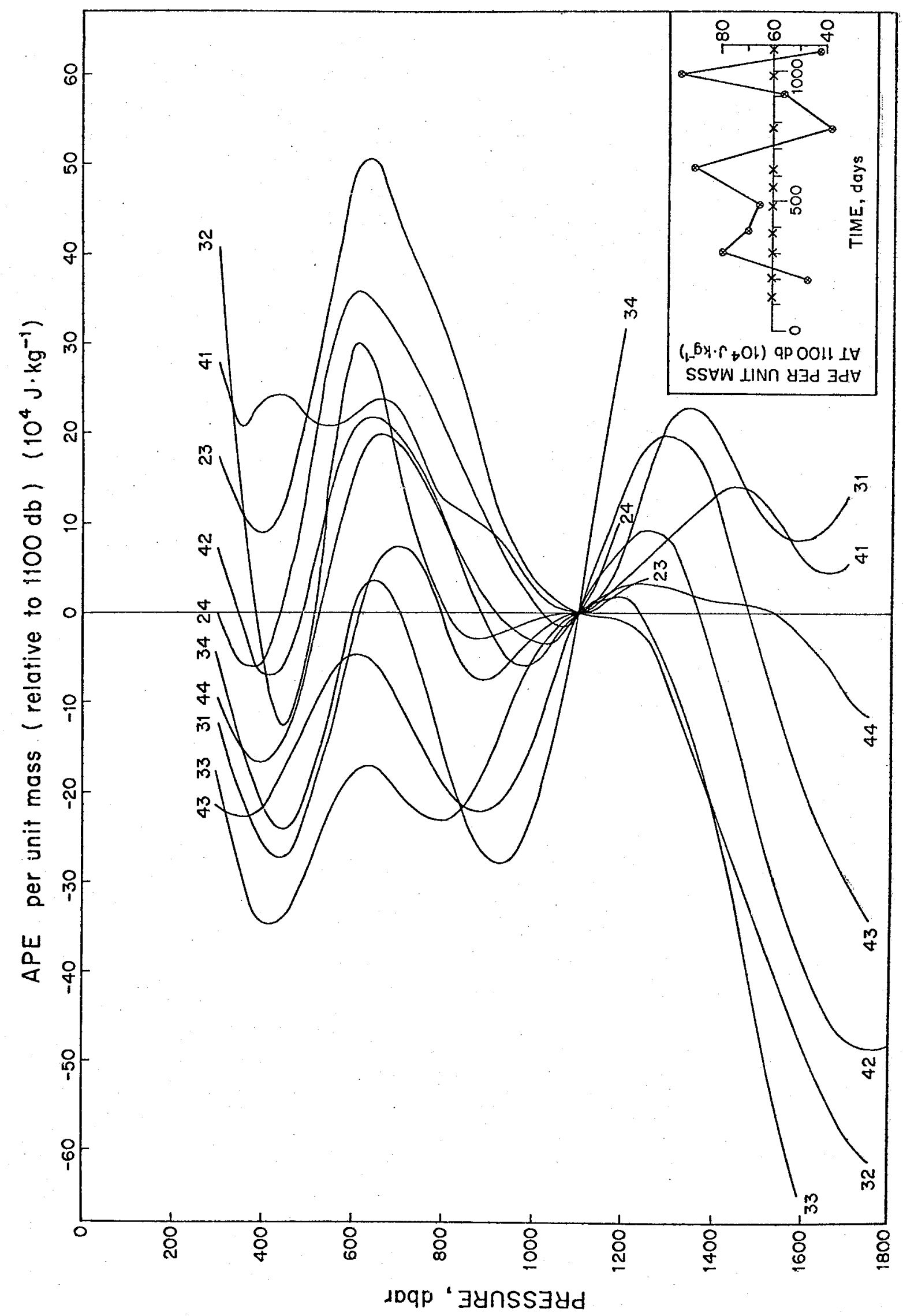




\subsection{Discussion}

From this analysis of the Bay of Biscay observations the following conclusions may be drawn:

1) There is a well defined mean field in salinity anomaly in the Bay of Biscay between depths of 400 and $1800 \mathrm{~m}$. The horizontal structure is dominated by a strong meridional gradient with a maximum value of $-.42 \times 10^{-3} \mathrm{ppt} \mathrm{km}^{-1}$ at $1200 \mathrm{db}$; there is a weaker zonal gradient with maximum value of $-.12 \times 10^{-3}$ ppt $\mathrm{km}^{-1}$ at the same depth and zonal curvature with a maximum value of $-.5 \times 10^{-6}$ ppt $\mathrm{km}^{-2}$ also at $1200 \mathrm{db}$.

2) Departures from the mean horizontal structure below the thermocline are seasonal and result from changes in the zonal structure, evidenced by changes in the zonal curvature term. The resultant change in salinity anomaly at a given location is 0 ( $\pm 25 \mathrm{ppm})$.

3) The reference specific volume field oscillates seasonally with an amplitude of about $1 \times 10^{-5} \mathrm{~cm}^{3} \mathrm{gm}^{-1}$, which corresponds to a vertical displacement of the thermocline of $\pm 15 \mathrm{~m}$. The scale of this motion is not determined by the observations; but must be larger than the spatial extent of the data, since the reference field is independent of horizontal position.

4) The vertical structure of APE per unit mass consists of two maxima: one at the thermocline, and one of the same approximate magnitude at about $1400 \mathrm{db}$. The thermocline APE is of the order of $70 \times 10^{-4} \mathrm{~J} \mathrm{~kg}^{-1}$. The amplitude and the vertical structure of APE change between realizations. That change is not demonstrably seasonal, although there is visual similarity between cruises which occurred at the same time of the year. The unresolved questions raised by the analysis of observations are: 
1) What are the horizontal scales of the seasonal variations in salinity anomaly and density?

2) What causes the observed seasonal vaxiations?

3) Should corresponding signals be expected in vertical displacement and hence in APE?

In Chapter 3 a theóretical model of oceanic response to atmospheric forcing is constructed to assist in the interpretation of the observational results. The model explores the consistency of seasonal windstress forcing as a mechanism for creating the observed seasonal signals. The model reproduces the observations, and provides a means for extrac-ting additional information from the observations. The windstress model was chosen in part because observations of the large scale winds are available. An alternate model might hypothesize that the observed seasonal signals result from wintertime convection in either the Labrador Sea or the Medicerranean Sea, both of which are sources of intermediate water in the Bay of Biscay. It is equally difficult to prove or disprove that hypothesis, since the amplitude of the convection, its horizontal structure and extent are unknown; neither is there information available about the expected phase relationship between the convection and its eventual influence on intermediate waters far from the convective source. The short horizontal scale of the salinity anomaly signal implied by the observations, and confirmed by further reduction of the data in Chapter 3 . argues against convection as a source, particularly if diffusion is important. However, lacking further observations, the hypothesis of a convective source remains untested: 
Chapter 3

\section{I Introduction}

The observation of seasonal signals in density and in salinity anomaly below the main thermocline introduces several questions which cannot be answered by the observations alone: What are the horizontal scales of these signals? What forces them? Why do they appear to be stronger below the thermocline? Should corresponding signals in vertical displacement of density surfaces and potential energy be expected? In this chapter an attempt to answer these questions is made through the construction of an analytical model: of atmospheric (windstress) forcing and oceanic response. The model is highly simplified, in order to be analytically tractable, but by hypothesis contains the essential physics of the problem. (That this is in fact true must be verified by comparison of the neglected terms with those retained.) The model, which follows that of Bryan and Ripa, 1978, assumes that the forcing consists of a waveform which may be propagating eastward or westward, or standing, and whose wavelength, phase, amplitude and frequency are parameters. The forcing acts upon the ocean, through a mixed layer of finite depth, as a body force rather than a stress. The model ocean is continuously stratified with depth variable buoyancy frequency $\mathrm{N}$ below a surface mixed layer; the Coriolis parameter is allowed to vary with latitude in the usual B-plane approximation. There is a meridional barrier at the eastern edge of the basin, and no bottom topography, friction or diffusion. The stratification, mixed layer depth and total depth are parameters. The oceanic response to the forcing consists of two parts: a forced wave of wavelength and frequency corresponding to the atmospheric forcing, and a sum of free waves required to satisfy the constraint of no flow into 
the boundary. The free waves are Rossby waves with meridional wavenumber equal to that of the forced wave, anc zonal wavenumber determined by the Rossby wave dispersion relation. The forcing scale at seasonal period is not well known, but it is certainly as large as the basin. Observational evidence of annual windstress forcing is discussed in section 4 of this chapter. The free response zonal scales are determined primarily by the stratification, which is well known. Once the dominant free wave scales have been established, the model can then predict the phase, horizontal and vertical displacements, the kinetic and potential energies of the system.

The predicted horizontal displacements, acting on a tracer field with mean horizontal gradients, will deform the initial tracer field (reversibly, in the absence of friction and diffusion) so that the progress of the disturbance through the fluid is traced by the anomaly in horizontal structure. Using the observed mean gradients of salinity anomaly $(\Delta \mathrm{s})$, the model predicts the amplitude and phase of the signal in $\Delta \mathrm{s}$. In order to compare the result with the observed salinity anomaly in section 4 , the data are fit to a progressive wave of set wavelength and frequency, and the amplitude and phase calculated. In terms of particle velocities, the dominant oceanic response away from the coast is the first free baroclinic wave, so only that wave is used in the fit to the observations. A similar analysis is pexformed using the observed vertical displacements. 


\subsection{Model}

The governing momentum equations are:

$$
\begin{aligned}
& \frac{\partial u}{\partial t}-f v=-\frac{1}{\rho_{0}} \frac{\partial p}{\partial x}+x \\
& \frac{\partial v}{\partial t}+f u=-\frac{1}{\rho_{0}} \frac{\partial p}{\partial y}+y
\end{aligned}
$$

with $\mathrm{x}$ and $\mathrm{y}$ positive eastward and northward co-ordinates, $\mathrm{u}$ and $\mathrm{v}$ the eastward and northward velocities, $p$ the pressure and $X$ and $Y$ body forces which parameterize the effect of the windstress through the mixed layer. The fluid is assumed to be hydrostatic; so that

$$
\frac{\partial p}{\partial z}=-\rho g
$$

with $z$ the vertical co-ordinate, which decreases with depth frorn a value of zero at the surface. It is also assumed that the fluid is incompressible, and that density is conserved following fluid parcels. The equation of continuity is then

$$
\frac{\partial u}{\partial x}+\frac{\partial v}{\partial y}+\frac{\partial w}{\partial z}=0
$$

The density $\rho$ is presumed to be constant through the mixed layer but a function of $x, y, z$ and $t$ below:

$$
\begin{aligned}
& \rho=\bar{\rho}(z)+\rho^{\prime}(x, y, t) \\
& \rho=\rho_{0}=\text { constant } \quad 0>z>-h
\end{aligned}
$$




$$
\rho=\rho_{0}+\frac{\rho_{\mathrm{o}}}{\gamma g} \cdot \mathrm{N}_{\mathrm{o}}^{2}\left[\mathrm{e}^{\gamma \mathrm{h}}-\mathrm{e}^{\gamma \mathrm{z}}\right] \quad-\mathrm{h}>\mathrm{z}>-\mathrm{H}
$$

where $h$ is the depth of the mixed layer and $H$ is the depth of the fluid. This distribution of density results in a simple buoyancy frequency profile:

$$
N^{2}=-\frac{g}{\rho_{0}} \frac{\partial \bar{\rho}}{\partial z}=N_{0}^{2} e^{\gamma z}
$$

which is analytically tractable. The density equation may then be written as :

$$
\frac{\partial \rho}{\partial t}-\frac{\rho O N^{2}}{g} \dot{w}=0
$$

where the horizontal advection of density has been neglected; $w$ is the vertical velocity.

A vorticity equation may be derived by subtracting the derivative with respect to $y$ of (3.1a) from the derivative with respect to $x$ of (3.1b).

$$
\frac{\partial}{\partial t}\left(\frac{\partial v}{\partial x}-\frac{\partial u}{\partial y}\right)-f \frac{\partial w}{\partial z}+\beta v=\frac{\partial y}{\partial x}-\frac{\partial x}{\partial y}
$$

From the density equation (3.4), and using (3.1c), the following expression for the vertical velocity may be derived:

$$
\frac{\partial w}{\partial z}=-\frac{\partial}{\partial t}\left[\frac{\partial}{\partial z} \frac{I}{\rho_{0} N^{2}} \frac{\partial p}{\partial z}\right]
$$


If the geostrophic streamfunction $p$ is defined by

$$
\begin{aligned}
& \rho_{0} f v=\frac{\partial p}{\partial x} \\
& \rho_{0} f u=-\frac{\partial p}{\partial y}
\end{aligned}
$$

then (3.2) may be written in terms of $p$ :

$$
\frac{\partial}{\partial t}\left[\nabla_{H}^{2}-\lambda^{2}\right] p+\beta \frac{\partial p}{\partial x}=f_{0}\left[\frac{\partial Y}{\partial x}-\frac{\partial x}{\partial y}\right]
$$

with $\nabla_{H}^{2}$ the horizontal laplacian operator $\frac{\partial^{2}}{\partial x^{2}}+\frac{\partial^{2}}{\partial y^{2}}$, and $\lambda^{2}$ a constant.

Equation (3.4) holds provided

$$
\frac{\partial}{\partial z}\left[\frac{f^{2}}{N^{2}} \frac{\partial p}{\partial z}\right]-\lambda^{2} p=0
$$

which allows the separation of the solution $p$ into vertical and horizontal structure functions. Thus solutions of (3.4) and (3.5) are sought in the form:

$$
p=\hat{p}(z) Q(x, y)
$$

The boundary conditions imposed are

$$
\begin{aligned}
& \mathrm{u}=0 \text { at } \mathrm{x}=0 \\
& \mathrm{w}=0 \text { at } \mathrm{z}=0,-\mathrm{H} \\
& \mathrm{w}, \mathrm{p} \text { continuous across } \mathrm{z}=-\mathrm{h} .
\end{aligned}
$$

The windstress forcing is assumed to have the form 
$X, Y=\hat{x} \sin \ell y, \hat{y} \cos \ell y) \exp \left(i\left(k_{f} x-\omega t\right)\right)$

The response may be expressed as a sum of a forced wave and an infinite number of free waves:

$$
\begin{gathered}
p=\alpha_{f} \hat{p}_{f}(z) \cos l y \exp \left[i\left(k_{f} x-\omega t\right)\right]+ \\
\sum_{n=0}^{\infty} \alpha_{n} \hat{p}_{n}(z) \cos l y \exp \left[i\left(k_{n}-\omega t\right)\right]
\end{gathered}
$$

The amplitudes $\alpha_{n}$ are determined by application of the boundary condition at $x=0$. The vertical structure function $\hat{p}_{f}(z)$ of the forced wave is determined from (3.5) with $\lambda$ given by the forcing parameters according to $(3.4)$ :

$$
\lambda_{f}^{2}=\frac{\beta k_{f}}{\omega}-k_{f}^{2}+\ell^{2}
$$

The structure functions $\hat{p}_{n}(z)$ of the free waves are determined from (3.5) for discrete values of $\lambda_{n}^{2}$ which satisfy the constraint of continuous pressure across the base of the mixed layer.

The vertical equation (3.5) can be rewritten in terms of the transformed $z$-variable $s=\exp \left(\gamma_{z} / 2\right)$ :

$$
\begin{aligned}
& s^{2} \hat{p}^{\prime}-s \hat{p}^{\prime}+s^{2} \mu^{2} p=0 \quad-h>z>-H \\
& \mu^{2}=\left[\frac{2 N(-h) \lambda}{f \gamma}\right]^{2}
\end{aligned}
$$


which can be solved in terms of first order Bessel functions. Since the boundary conditions on the vertical equation are easily expressed in terms of vertical velocity, it is simplest to relate pressure to vertical velocity $w$ and solve the problem in $w$. In doing that the barotropic mode is excluded; in order to retain both the barotropic mode and the simple boundary conditions, solutions in $p$ and in $w$ will be used, and a consistent conversion between them established. This procedure departs from that used by Bryan and Ripa, who ignore the barotropic wave altogether. Pressure and vertical velocity are related by:

$$
\frac{\partial w}{\partial z}=-\frac{\partial}{\partial t} \frac{\partial}{\partial z}\left(\frac{1}{N^{2} \rho_{0}} \frac{\partial p}{\partial z}\right)
$$

or

$$
\hat{w}_{z}=\frac{-i \omega \lambda^{2}}{f^{2} \rho_{0}} \hat{p}=-\lambda^{2} A^{-1} \hat{p}_{i} \quad \AA=\frac{f^{2} \rho_{0}}{i \omega}
$$

The equation for $\hat{w}$ is then

$$
s^{2} \hat{w}^{\mu}+s \hat{w}+s^{2} \mu^{2} \hat{w}=0
$$

with solutions of the form

$$
\hat{w}=c_{n} \zeta_{0}\left(\mu_{n} s\right)=c_{n}\left[J_{0}\left(\mu_{n} s\right)+b_{n} Y_{0}\left(\mu_{n} s\right)\right]
$$

$J_{0}$ and $y_{0}$ Bessel functions.

In addition to boundary conditions at the top and bottom, the vertical velocity and its first derivative are constrained to be continuous across $z=-h$. The mixed layer solution for $\hat{w}_{n}$ is simply proportional to $z$. Applying the constraint on $w$ at $z=-h$, the solution for 
$\hat{w}_{n}$ is:

$$
\begin{array}{ll}
\hat{w}_{n}=-z c_{n} \oint_{0}\left(\mu_{n} s^{*}\right) / h & 0>z>-h \\
\hat{w}_{n}=c_{n} \oint_{0}\left(\mu_{n} s\right) & h>z>-H \\
s^{*}=s(-h)=\exp [-\gamma h / 2] &
\end{array}
$$

The condition $w=0$ at $z=-H$ is satisfied separately by each mode if

$$
\begin{aligned}
& b_{n}=\frac{-J_{0}\left(\mu_{n} \varepsilon\right)}{Y_{0}\left(\mu_{n} \varepsilon\right)} \\
& \varepsilon=s(-H)=\exp [-\gamma H / 2]
\end{aligned}
$$

The eigenvalues $\mu_{n}$ are determined by application of the constraint on $\hat{w}_{z}$ at $z=-h$ :

$$
\zeta_{0}\left(\mu_{n} s^{*}\right)=\left(\frac{r h}{2}\right) \mu_{n} s^{*} \zeta_{1}\left(\mu_{n} s^{*}\right)
$$

The condition of continuous $\hat{w}_{z}$ across $z=-h$ is equivalent to continuous p. Using (3.8) the solution in terms of $\hat{p}$ is:

$$
\begin{array}{ll}
\hat{p}_{n}=\frac{-A}{\lambda_{n}^{2}} \frac{c_{n}}{h} \oint_{0}\left(\mu_{n} s^{*}\right) & 0>z>-h \\
\hat{p}_{n}=\frac{A}{\lambda_{n}^{2}} \frac{\mu_{n} \gamma}{2} c_{n} s \xi_{1}\left(\mu_{n} s\right) & -h>z>-H
\end{array}
$$


The eigenfunctions $\hat{p}_{n}$ are orthonormal, and the normalizing constant determines $c_{n}$; that is,

$$
\int_{\varepsilon}^{s^{*}} \frac{\hat{p}_{n} \hat{p}_{m}}{s} d s=\delta_{m n}
$$

(with $\delta_{\mathrm{mn}}$ the Kroenecker delta) implies that

$$
\frac{1}{c_{n}^{2}}=\left[\frac{\mu n \gamma}{2 \lambda_{n}^{2}}\right]^{2} A^{2}\left[\frac{s^{2}}{2}\left\{\xi_{1}^{2}\left(\mu_{n} s\right)+\zeta_{0}^{2}\left(\mu_{n} s\right)-\frac{2}{\mu_{n} s} \oint_{0}\left(\mu_{n} s\right) \zeta_{1}\left(\mu_{n} s\right)\right\}\right]_{\varepsilon}^{s^{*}}
$$

for $n \geq 1$, and

$$
\frac{1}{c_{0}^{2}}=.5 \gamma(H-h)
$$

For the forced wave, if the wave is eastward-moving.$\lambda_{f}^{2}$ and therefore $\mu_{f}^{2}$ are negative and the solution for $\hat{w}_{f}$ is in terms of modified Bessel functions

$$
\begin{array}{rlr}
\hat{w}_{f_{+}}=-z c_{f} \mathcal{F}_{O}\left(\mu_{f} s^{*}\right) / h & 0>z>-h \\
\hat{w}_{f_{f}}=c_{f} \mathcal{F}_{O}\left(\mu_{f} s\right) & -h>z>-H \\
\mathcal{J}_{O}\left(\mu_{f} s\right)=\left[I_{O}\left(\mu_{f} s\right)+b_{f} K_{O}\left(\mu_{f} s\right)\right] &
\end{array}
$$

For a westward moving forced wave the solution is

$$
\hat{w}_{f-}=-z c_{f} \zeta_{0}\left(\mu_{f} s^{*}\right) / h \quad 0>z>-h
$$




$$
\hat{w}_{f-}:=c_{f-} \zeta_{o}\left(\mu_{f} s\right)
$$

The constants $\mathrm{b}_{f_{+}}$and $\mathrm{b}_{f_{-}}$are determined by requiring that $\hat{w}_{f}$ vanish at $z=-H$. The coefficients $c_{f_{+}}$and $c_{f_{-}}$are determined by assuming that the vertical velocity at the base of the mixed layer due to the wind is given by the divergence of the Ekman mass flux within the mixed layer:

$$
\hat{w}(-h)=\frac{\operatorname{curl} \tau}{\rho_{0} f}=c_{f}\left[\mathcal{F}_{O}\left(\mu_{f}^{s^{*}}\right), \hat{\zeta}_{O}\left(\mu_{f^{*}} s^{*}\right)\right]
$$

The coefficients $c_{f}$ are further adjusted so that the amplitudes of $w_{f_{+}}$and $w_{f_{-}}$are equal at the base of the mixed layer. Thus

$$
\begin{aligned}
& \hat{w}_{f}=\frac{\operatorname{curl} \tau}{\rho_{O} f \mathcal{F}_{O}}\left(\mu_{f} s^{*}\right) \mathcal{F}_{O}\left(\mu_{f} s\right) \\
& \hat{w}_{f}=\frac{\operatorname{curl} \tau}{\rho_{O} f \mathcal{F}_{O}}\left(\mu_{f^{s}} s^{*}\right)\left[\frac{\mathcal{F}_{O}\left(\mu_{f^{s}}\right)}{\zeta_{O}\left(\mu_{f^{*}} s^{*}\right.}\right] \zeta_{O}\left(\mu_{f} s\right)
\end{aligned}
$$

The forced wave streamfunctions corresponding to (3.17) are:

$$
\begin{aligned}
& \hat{p}_{f_{+}}=\frac{A}{\lambda_{f_{+}}^{2}} c_{f_{+}} s \mathcal{F}_{I}\left(\mu_{f} s\right) \cdot \frac{\mu_{f} \gamma}{2} \\
& \hat{p}_{f}=\frac{-A}{\lambda_{E}^{2}} c_{f} s \oint_{I}\left(\mu_{f} s\right) \cdot \frac{\mu_{f} \gamma}{2}
\end{aligned}
$$

where

$$
\mathcal{F}_{v}=\operatorname{Re}\left[I_{v}+e^{i \pi \nu} k_{v}\right]
$$


Finally, in order to determine the amplitudes $\alpha_{n}$ of the free waves the boundary condition at $x=0$ is applied:

$$
u(x=0)=0
$$

or

$$
\ell \cdot \sin \ell_{y} \hat{p}_{f}(z)=-\sum_{h=0}^{\infty} \ell \text { sinly } \alpha_{n} \hat{p}_{n}(z)
$$

Applying the orthogonality condition (3.13):

$$
\alpha_{n}=-\int_{\varepsilon}^{s^{*}} \frac{\hat{p}_{f} \hat{p}_{n}}{s} d s
$$

which can be evaluated analytically (Abramowitz and Stegun, 1972, p. 484). The solution depends upon the direction of the forcing; for the eastward propagating wave:

$$
\begin{aligned}
& \alpha_{n}=\frac{c_{f} c_{n} \mu_{f} \mu_{n} \gamma^{2} A^{2}}{4 \lambda_{f}^{2} \lambda_{n}^{2}} s \mu_{n} \zeta_{2} \mathcal{F}_{1}+s \mu_{f} \zeta_{I} \mathcal{F}_{2} s * \\
& \alpha_{0}=\frac{c_{O} c_{E} \gamma \mu_{E} A}{2 \lambda_{E}^{2}}\left[\mathcal{F}_{0}\left(\mu_{F} s\right)\right]_{E}^{s *}
\end{aligned}
$$

(where subscript zero refers to the barotropic mode) while for the westward propagating wave

$$
\begin{aligned}
& \alpha_{n}=\frac{c_{f} c_{n} \mu_{f} \mu_{n} \cdot \gamma^{2} A^{2} \cdot s \mu_{n} \zeta_{2}\left(\mu_{n} s\right) \zeta_{I}\left(\mu_{f} s\right)-s \mu_{f} \ell_{I}\left(\mu_{n} s\right) \zeta_{2}\left(\mu_{f} s\right)}{4 \lambda_{f}^{2} \cdot \lambda_{n}^{2}} \mu_{n}^{2}-\mu_{f}^{2} \\
& \alpha_{0}=\frac{c_{0} c_{f} \gamma \mu_{f} A}{2 \lambda_{f}^{2}}\left[\zeta_{f}\left(\mu_{f} s\right)\right]_{\varepsilon}^{s *}
\end{aligned}
$$


The boundary condition of no zonal velocity at $x=0$ assures that there is no energy flux into the boundary. As $x$ approaches $-\infty$, the energy from the free waves, which exist only because of the presence of the meridional barrier, inst be propagating away from the barrier, or westward. This radiation condition requires that only the free waves with westward group velocity be included in the solution. The dispersion relation is a quadratic in $k$, so that two solutions are possible for each $\lambda_{n^{*}}$ The shorter wave has eastward group velocity, the longer wave westward group velocity. The long waves are therefore chosen for the solution. 


\subsection{Model results}

The parameters used in the calculation are listed in Table 3.1 Discussion of the windstress obsexvations which were used in choosing the forcing parameters is deferred to section 4. The mixed layer depth, total depth and the stratification parameters are taken from the Bay of Biscay observations. The eigenvalues of the first four modes were calculated using a Newton-Raphson iteration of (3.12); they are tabulated in Table 3.2. For $\mathrm{n}=2$ and higher, the modes are trapped within $20 \mathrm{~km}$ of the coast. (Recall that the observations extend roughly $700 \mathrm{~km}$ from the coast.) The dispersion relation is:

$$
k_{n}=\frac{-\beta}{2 \omega} \pm \frac{1}{2} \sqrt{\frac{\beta^{2}}{\omega^{2}}-4\left(\lambda_{n}^{2}+\ell^{2}\right)}
$$

For modes with $n \geq 2 \mathrm{k}_{\mathrm{n}}$ has two complex roots; the root which decays away from the coast must be chosen. Because the higher modes are strongly trapped only the barotropic and first baroclinic modes are included in the calculations which follow. The wavelengths and phase speeds of these modes are also given in Table 3.2. The first baroclinic wave has a wavelength of $391 \mathrm{~km}$. The barotropic wave is much longer than the basin and therefore has negligible displacements. The vertical and horizontal displacements associated with the first baroclinic wave are much larger than those associated with the forced wave and the barotropic wave. As a result the effective difference in terms of displacements between propagating and standing windstress forcing is the amplitude of the free response: for a standing wave the location of the nodes of the wave pattern are important in determining the response amplitude. Since the horizontal structure of the annual windstress is not well known, the eastward propagating wave was chosen as representative and the 
TABLE 3.1

Model Parameters

\begin{tabular}{|c|c|c|}
\hline Parametes & & Description \\
\hline $\mathrm{h}$ & : & Mixed layer depth \\
\hline $\mathrm{H}$ & : & Total depth \\
\hline $2 \pi / \mathrm{N}_{0}$ & : & Buoyancy period at $h$ \\
\hline $1 / \gamma$ & : & e-folding depth for stratification \\
\hline $2 \pi / k_{F}$ & $:$ & Zonal wavelength of forcing \\
\hline $2 \pi / l$ & $:$ & Meridional wavelength of forcing \\
\hline $2 \pi / \omega$ & $:$ & period \\
\hline$\beta$ & & Beta \\
\hline$f$ & $:$ & Coriolis parameter \\
\hline$\tau^{Y}$ & : & Meridional wind stress \\
\hline$\tau^{x}$ & : & Zonal windstress \\
\hline
\end{tabular}

Value

$400 \mathrm{~m}$

$5000 \mathrm{~m}$

$1.35 \times 10^{3} \mathrm{sec}$

$1300 \mathrm{~m}$

$12000 \mathrm{~km}$

$6000 \mathrm{~km}$

1 year

$1.6 \times 10^{-11}\left(\mathrm{~m} \mathrm{sec}^{-1}\right.$

$$
10^{-4} \sec ^{-1}
$$

10. $\times 10^{-2}$ pascal

$-4 . \times 10^{-2}$ pascal 
TABLE 3.2

Eigenvalues and Corresponding Wavelengths and Phase Speeds

$\begin{array}{cccc}\text { MODE } & \mu_{\mathrm{n}} & 2 \pi / \mathrm{k} & \mathrm{\omega} / \mathrm{k} \\ \text { forced } & .647 & \mathrm{~km} & \mathrm{~m} \mathrm{sec}^{-1} \\ \text { Barotropic Mode } & .049 & 12000 & -13.0 \\ \text { Baroclinic Modes } & & -4.1 \times 10^{5} & \\ 1 & 3.59 & & -.0125 \\ 2 & 7.57 & -391 & \\ 3 & 11.7 & & \\ 4 & 16.0 & & \end{array}$


results which follow are for that case:

The streamfunctions $\hat{p}_{f}, \hat{p}_{0}, \hat{p}_{1}$ are plotted in Fig. 3.1 , illustrating the relative amplitudes of the barotropic and baroclinic components. Recall that the boundary condition at $\mathrm{x}=0$ requires that the total streamfunction vanish there. As a result, no signal in sea level at the coast is expected.

Displacement amplitudes $\zeta$ as a function of depth were calculated

as

$$
\frac{d \hat{\zeta}}{d t}=\hat{w}(z)
$$

or

$$
\hat{\zeta}=\frac{-\hat{w}}{i \omega}
$$

and are plotted in Fig. 3.1 for both the forced and the first free modes. The meridional velocity amplitude

$$
\hat{v}=k \hat{p}(z)
$$

is plotted in Fig. 3.3, for the forced and first baroclinic modes. The meridional velocity. of the barotropic mode is two orders of magnitude smaller than that of the forced mode.

Since the free wave zonal velocity is much smaller than the meridional velocity, the salt conservation equation with no diffusion is approximately

$$
\frac{\partial S}{\partial t}+v(x) \frac{\partial S}{\partial y} \stackrel{\simeq}{=}
$$

or 
Figure 3.1 Streamfunction $\hat{p}$ for eastward forced wave and corresponding barotropic and first baroclinic free waves. 


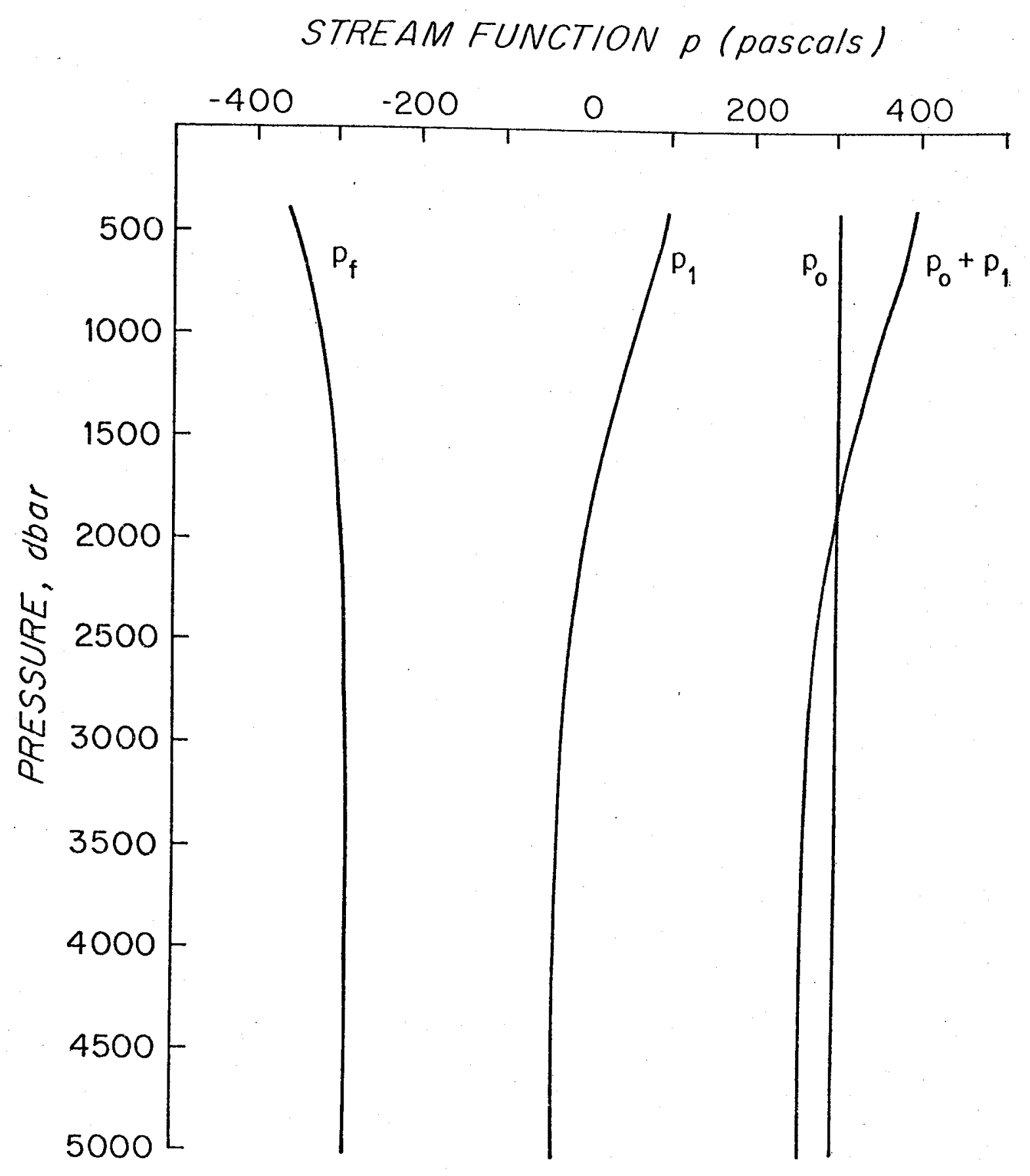


Figure 3.2 Vertical displacements $\hat{\zeta}$ for eastward forced wave and corresponding first baroclinic wave. 


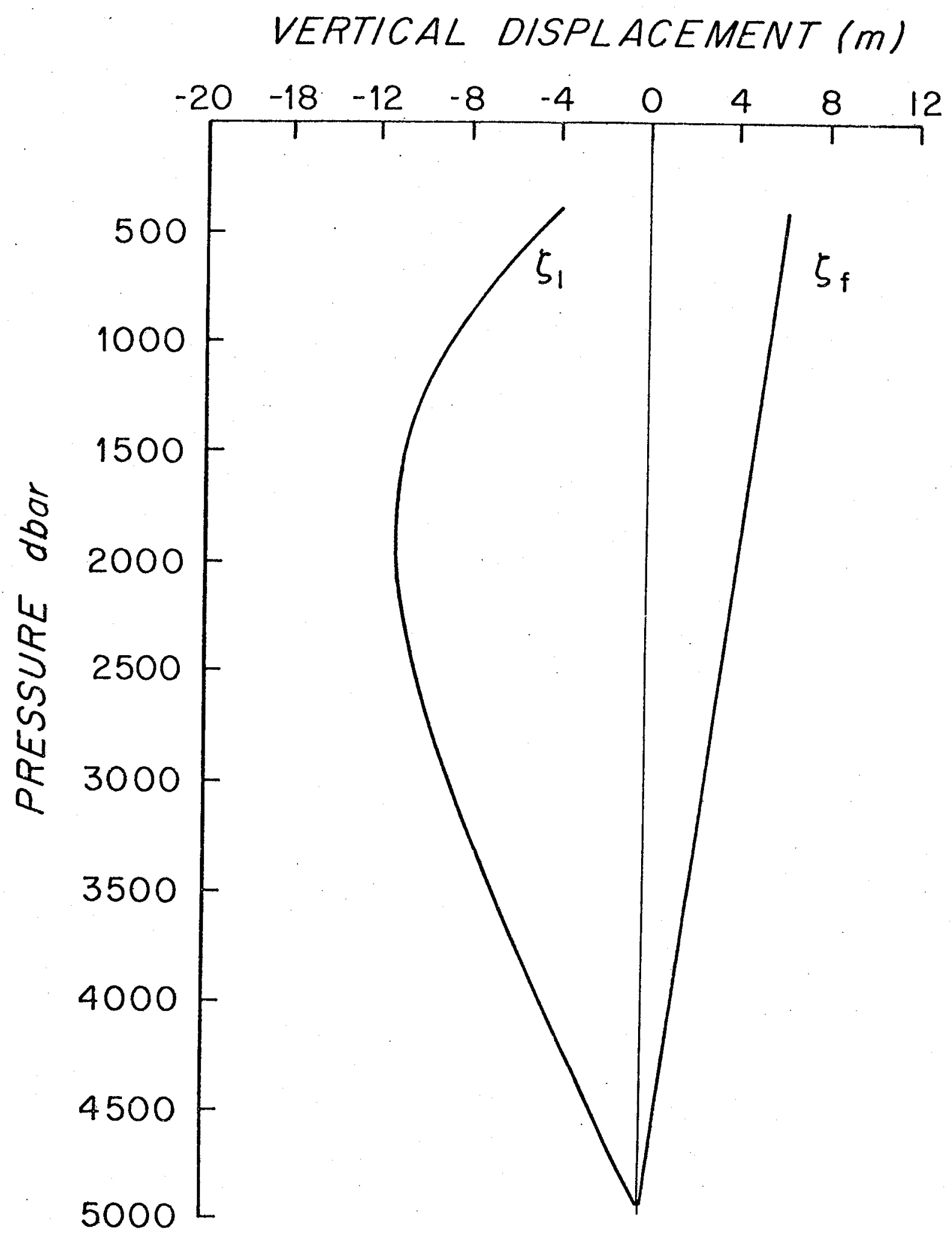


Figure 3.3 Meridional velocity $\hat{v}$ for eastward forced wave and corresponding first baroclinic wave. 


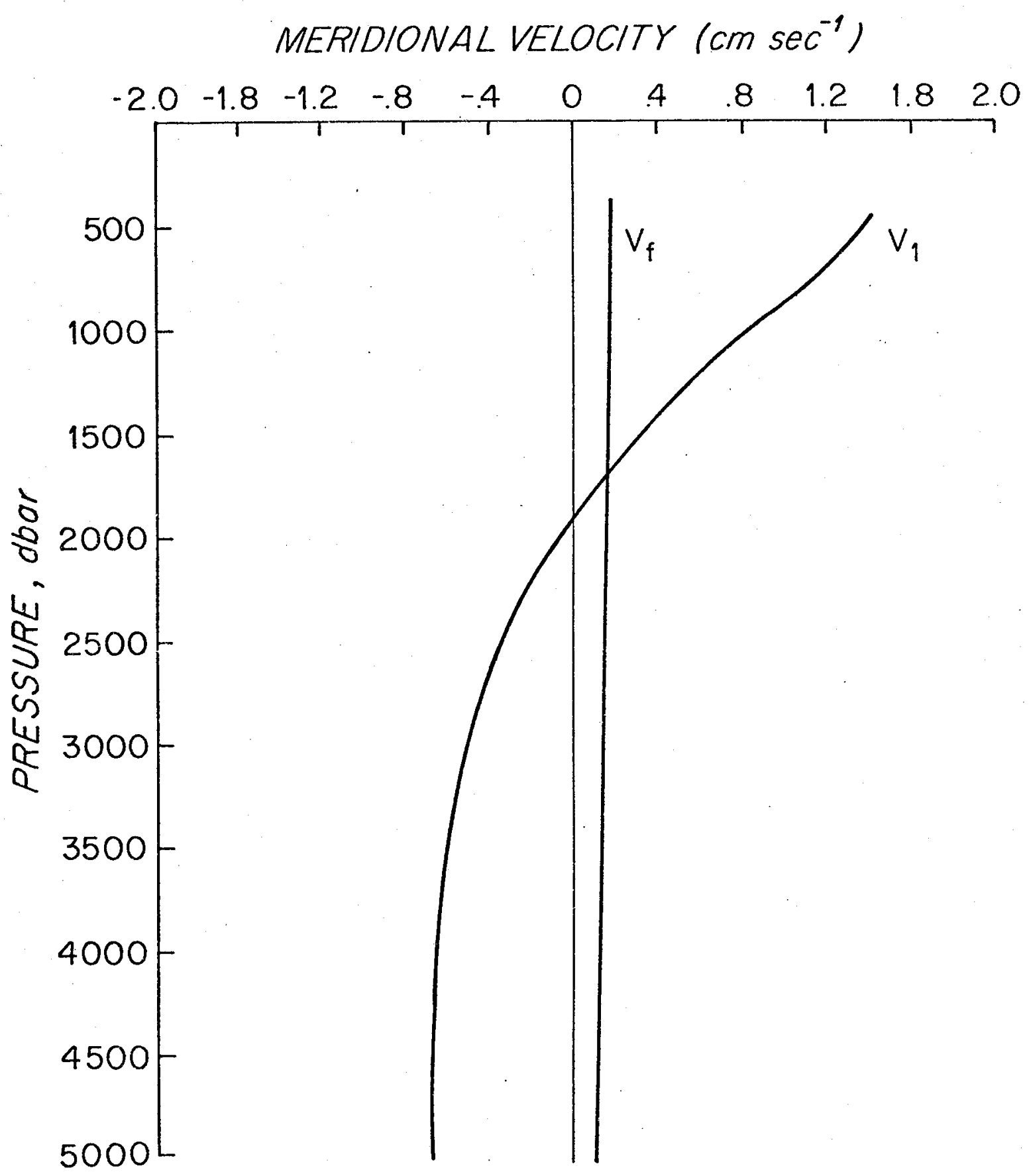




$$
\begin{gathered}
s(x, y, z, t)=s_{0}(x, \eta, 0) \cdot \hat{s}(z) \\
\eta=y-\int_{0}^{t} v d t
\end{gathered}
$$

For a simple initial field $s_{o}$, with salinity anomaly a linear function of $y$ and an arbitrary function of $x$ :

$$
s(x, y, z, t)=\left[s_{0}(x, y, 0)+s_{y} \cdot \int_{0}^{t} v d t\right] \hat{s}(z) .
$$

Using the observed values of $S_{y}$ from the Bay of Biscay data, one may obtain an estimate of the salinity anomaly variation amplitude:

$$
\hat{\Delta S}=-\left(S_{y}\right) \text { obs } \frac{\hat{v}(z)}{i \omega}
$$

which is plotted in Fig. 3.6 for those levels where $\left(s_{y}\right)$ obs is available.

Note that certain phase relationships predicted by the model may be tested. If the windstress varies as

$$
\begin{aligned}
& \tau \cong \cos \Phi+i \sin \Phi \\
& \Phi=k_{f} x-\omega t-\phi
\end{aligned}
$$

then the displacement and the salinity anomaly fields will vary as $(\cos \Phi+i \sin \Phi)$, whereas the velocity will be $90^{\circ}$ out of phase with the forcing. The observed phase $\phi$ in salinity anomaly and displacement can be compared to the observed phase of the annual windstress, as discussed in the following section. 


\subsection{Comparison of Model and Observations}

There are two concerns in this section: a discussion of the observation of windstress in the North Atlantic which were used to determine the choice of forcing parameters in the model and the phase of the forcing; and a further reduction of the oceanic observations of Chapter 2 which allows direct comparison of the model predictions and the observational results.

There exists a comprehensive meteorological data set for the North Atlantic from 1948 to 1972 (Bunker and Worthington, 1976). Charts of the average annual windstress over the North Atlantic were prepared by Bunker and Worthington; to estimate the amplitudes and wavelengths of the annual windstress forcing appropriate to this problem zonal and meridional sections of $\tau^{x}$ and $\tau^{y}$ (along $45^{\circ} \mathrm{N}$ and $10^{\circ} \mathrm{W}$ ) were taken from those charts (see Fig. 3.4). The entire 25 year record of windstress was examined for evidence of phase propagation, with no conclusive results. There is some evidence that the zonal windstress maximum shifts eastward in summer and fall relative to its position in winter and spring (Fig. 3.5). The forcing wavelengths used in the model were estimated from Fig. 3.5. The model results are not sensitive to the choice of forcing scales within reasonable limits. The forcing amplitudes were chosen such that the model $\Delta S$ response amplitude was approximately that given by the observations, with the ratio of $\tau^{x} / \tau^{y}$ as given by the wind observations held constant. The long term average amplitude of seasonal change in windstress estimated from the difference between winter and summer 25 year means is roughly $5 . \times 10^{-2}$ pascals $\left(.5\right.$ dyne $\left.\mathrm{cm}^{-2}\right)$ for $\tau^{\mathrm{x}}$ and $2 \times 10^{-2}$ pascals for $\tau^{Y}$ along $45^{\circ} \mathrm{N}$ between $10^{\circ}$ and $70^{\circ} \mathrm{W}$. One standard deviation of $\tau$ 
(a) Mexidional sections along $10^{\circ} \mathrm{W}$ and

(b) Zonal sections along $45^{\circ} \mathrm{N}$ of the annual average windstress components $\tau^{\mathrm{X}}$ and $\tau^{\mathrm{Y}}$. Values taken from Bunker and Worthington (1976). 


Figure 3.5 Seasonal averages using all the Bunker and Worthington (1976) data (1948-1972) of $\tau^{x}$ along $45^{\circ} \mathrm{N}$, by $10^{\circ}$ Marsden square. 


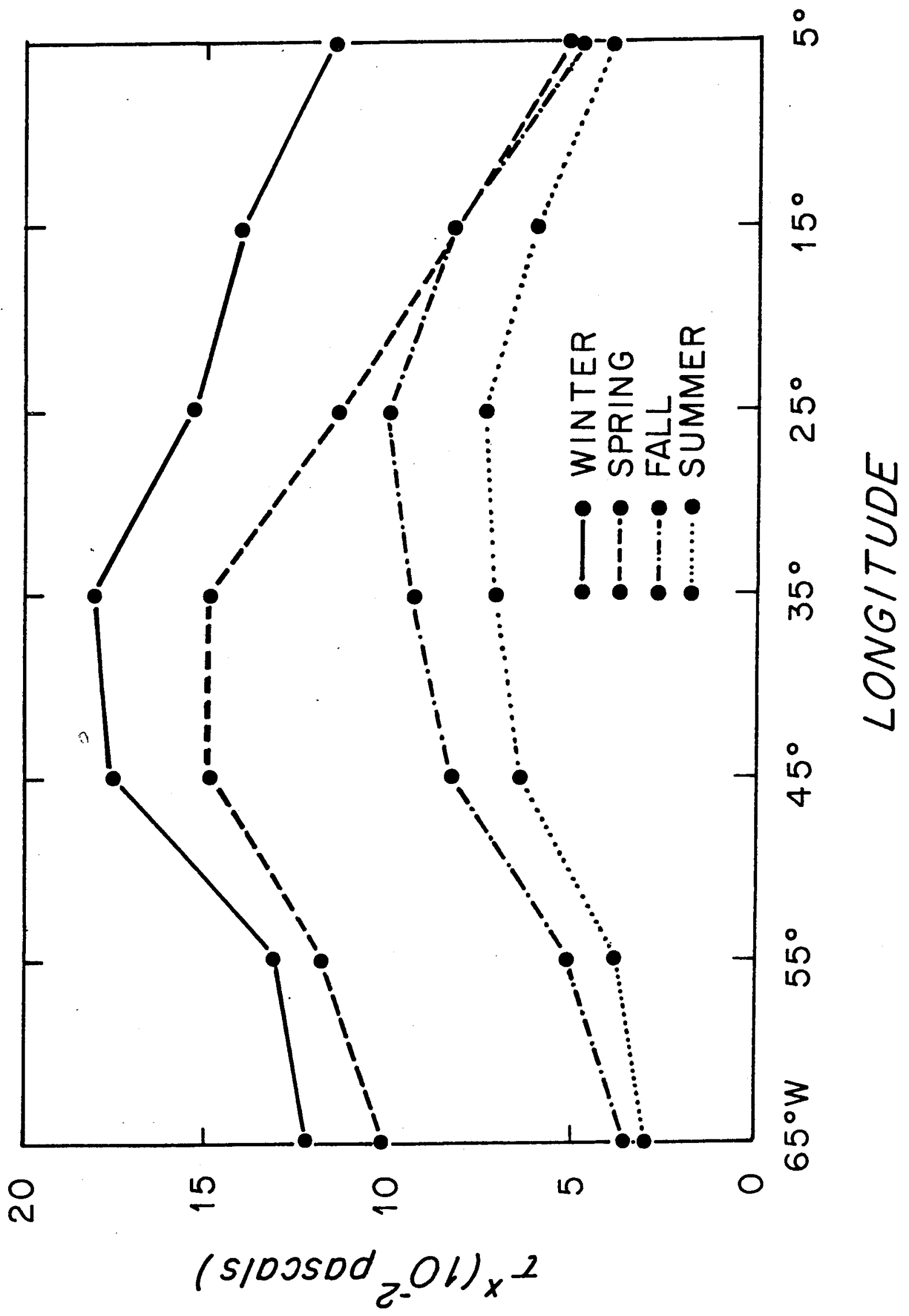


is 13. $\times 10^{-2}$ pascals and of $\tau^{Y} 9.7 \times 10^{-2}$ pascals for the winter season in the ten-degree Marsden Square corresponding to $40^{\circ}$ to $50^{\circ} \mathrm{N}$, $0^{\circ}$ to $10^{\circ} \mathrm{W}$. The standard deviations there for the summer season are $5.2 \times 10^{-2}$ pascals and $3.1 \times 10^{-2}$ pascals for $\tau^{x}$ and $\tau^{y}$ respectively. Similar variances are found along $45^{\circ} \mathrm{N}$ between $10^{\circ}$ and $70^{\circ} \mathrm{W}$. The windstress amplitudes used in the model are $10 \times 10^{-2}$ and $-4 \times 10^{-2}$ pascals for $\tau^{\mathrm{x}}$ and $\tau^{\mathrm{y}}$, within a standard deviation of the 25-year means.

The phase of the seasonal cycle of windstress was calculated by Firing (1978) for different longitude bands. At $38^{\circ} \mathrm{N}$, between $30^{\circ} \mathrm{W}$ and $40^{\circ} \mathrm{W}$, he calculated that the maximum in windstress occurred 55 days from January.

The model predicts westward propagating free disturbances of dominant wavelength $391 \mathrm{~km}$, frequency $I$ cpy and phase corresponding to a maximum at 55 days from 1 January. In Chapter 2 the data were fitted to polynomials in $x$ and $y$ to study the horizontal structure of salinity anomaly and vertical displacement. That procedure is inappropriate for comparing the model and the observations, since the propagation of waves through the field results in a complex interaction of space and time information which cannot be sorted out by fitting to polynomials. Instead, the data were fitted to traveling plane waves of the form

$$
\cos (k x-\omega t-\phi) \text {. }
$$

In that analysis, the steady horizontal structure of the salinity anomaly field was removed. The residuals were then fitted to

$$
s=a_{1} \sin \left(k_{1} x-\omega t\right)+a_{2} \cos \left(k_{1} x-\omega t\right)
$$


with

$$
\begin{aligned}
\mathrm{k}_{1} & =2 \pi / 391 \mathrm{~km} \\
\omega & =2 \pi / 365 \text { days } \\
\mathrm{a}_{1} & =-\mathrm{a}_{\mathrm{s}} \sin \phi \\
\mathrm{a}_{2} & =\mathrm{a}_{\mathrm{s}} \cos \phi
\end{aligned}
$$

and $x$ measured as actual horizontal distance from the $600 \mathrm{~m}$ isobath along the eastern boundary. The $600 \mathrm{~m}$ isobath does not correspond to a meridional barrier; the implications of that choice for $\mathbf{x}=0$ are discussed in section 3.5 .

The regression was done using all cruises at each level, and separately by cruise. In order to determine the amplitudes with statistical confidence greater than 90 per cent, the best estimate of phase was used for $\phi$, and the amplitude alone was fitted, with fixed phase.

The best estimate of $\phi$ corresponds to a maximum at 71 days from 1 January. The estimated error in the phase determination is $\pm .3 \mathrm{rad}$ (20 days), so that the calculated phase agrees with that of Firing within the errors of phase determination. The overall amplitude $a_{s}$ as a function of $z$ is plotted in Fig. 3.6, along with the amplitudes for cruises which show strong signals, and the model prediction for an eastward forced wave. Most of the variance in $a_{s}$ is found in four cruises: Phygas 23, 31, 42, 44; the others contribute very little signal. The lack of signal may be due in part to insufficient sampling, as there are significantly fewer stations in those cruises (see Table 2.1). For Phygas 42 the amplitude $a_{s}$ is positive, although it is plotted as -a in Fig. 3.7. The vertical structure is similar in the four cruises with large signals, and compares very well with the model prediction. 
Figure 3.6 Salinity anomaly amplitudes: heavy solid line is the model prediction, dased line is the overall value (excluding cruises 42 and 43) and the remaining thin lines are cruises $24,31,44$, and the negative of 42 , as noted. 
SALINITY ANOMALY AMPLITUDES, ppm

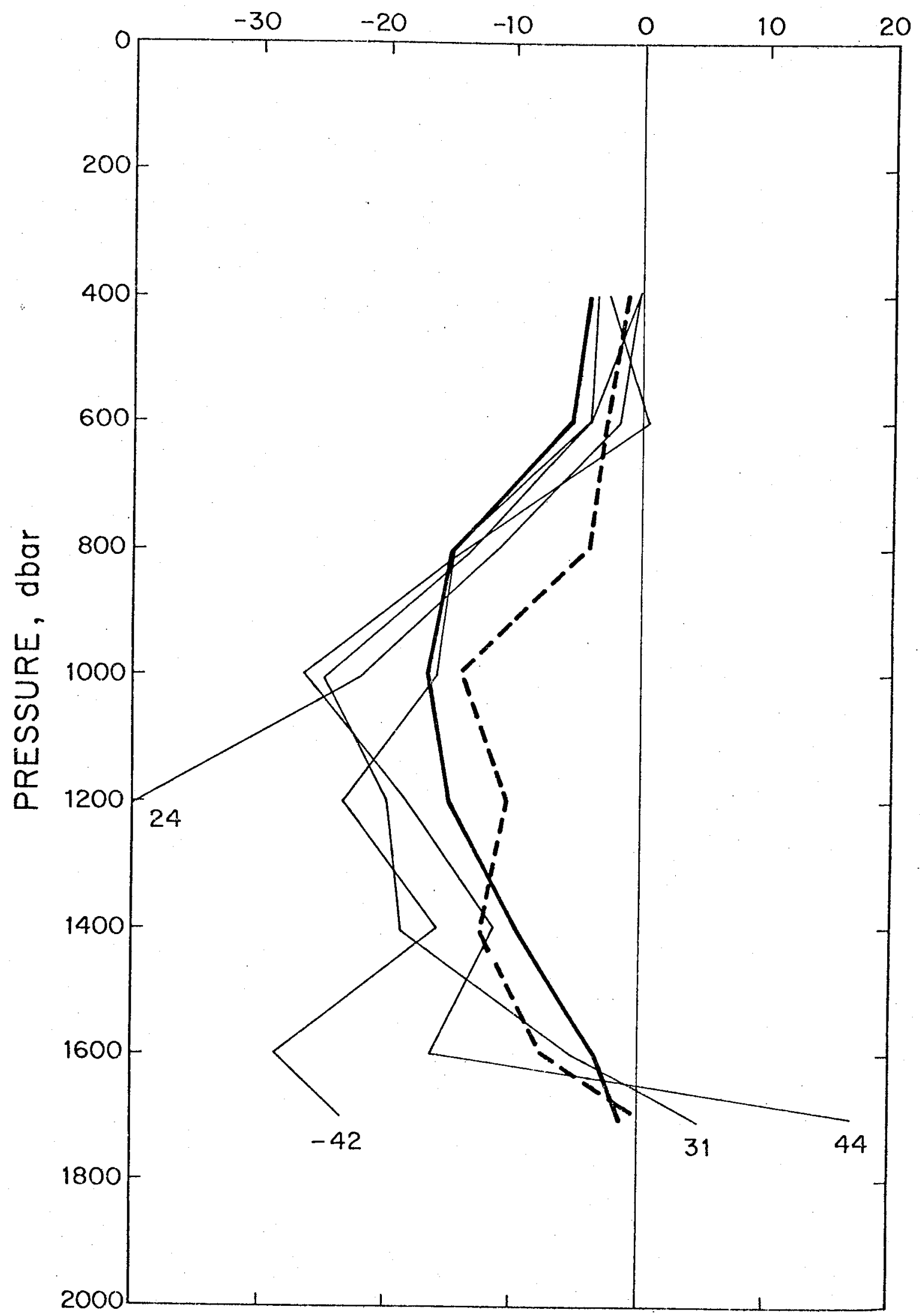


In order to determine how sensitive the regression fit is to the chosen parameters $k, \omega$, and $\phi$, tests were run using a range of values for each parameter, holding the other two at their chosen values. The ranges covered were

$2 \pi / \mathrm{k}(391 \mathrm{~km}): 300$ to $900 \mathrm{~km}$, steps of 100 and $200 \mathrm{~km}$ $2 \pi / \phi$ (12 months): 10 to 14 months; steps of 1 month $\phi-365 / 2 \pi$ (71 days) $: 50$ to 130 days, steps of 20 days

In all cases the ratio of the amplitude to its regression standard deviation was a maximum for the chosen parameters, and, with the exception of $\phi$ corresponding to 50 to 90 days, none other than the one with the three chosen parameters were significant at the 95 per cent confidence level (see Fig. 3.7). The estimated error in the determination of $\phi$ given above is taken from these results.

A similar analysis was carried out for the displacement field, although there were no identifiable mean horizontal gradients to be removed. The observed displacement amplitudes $a_{\pi}(z)$ are plotted in Fig. 3.8 along with the model prediction and values for Phygas 31 and 24 which showed the most consistent single cruise results.

The model predicts little seasonal change in APE per unit mass which is computed as an average over the area. Since the zonal extent of the data is longer than the zonal wavelength of dominant interior response, the predicted APE varies little with time. The model APE per unit mass does have vertical structure sinilar to that observed, with a maximum value at about $1400 \mathrm{db}$. The amplitude of the model APE is more than an order of magnitude smaller than observed; this is not surprising, since the root mean square displacements, calculated in Chapter 2, which include non-seasonal as well as seasonal variability, are 5 to 10 times as large 
Figure 3.7 Ratio of the least squares computed salinity anomaly amplitude $a_{s}$ to its standard deviation at 1000 db for ranges of wavelength, period and phase. Chosen parameters are circled. 

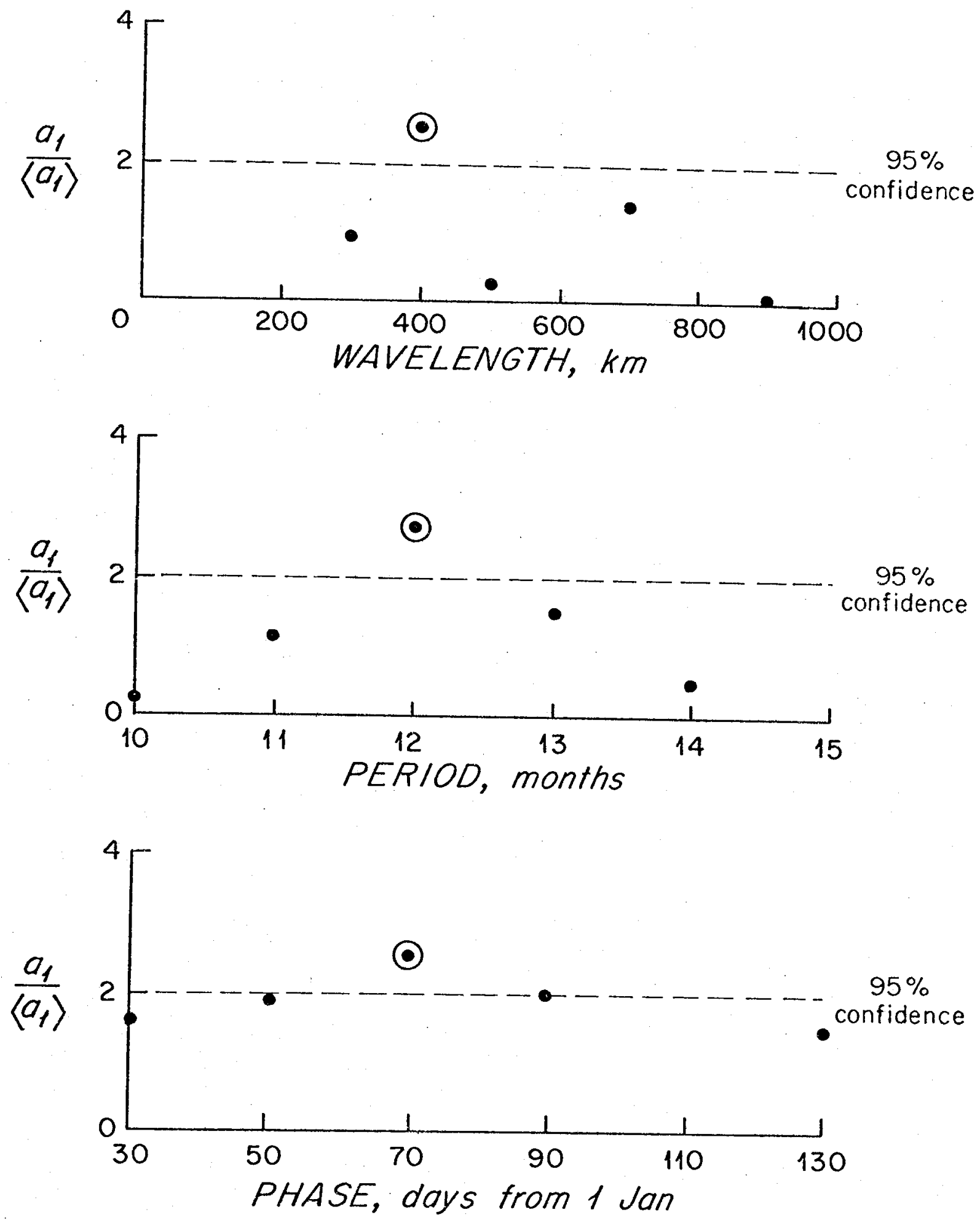
Figure 3.8 Displacement amplitudes: heavy solid line is the model prediction, dashed line is the overall value (excluding cruises 42 and 43 ) and the thin lines are cruises 31 and 24 . 


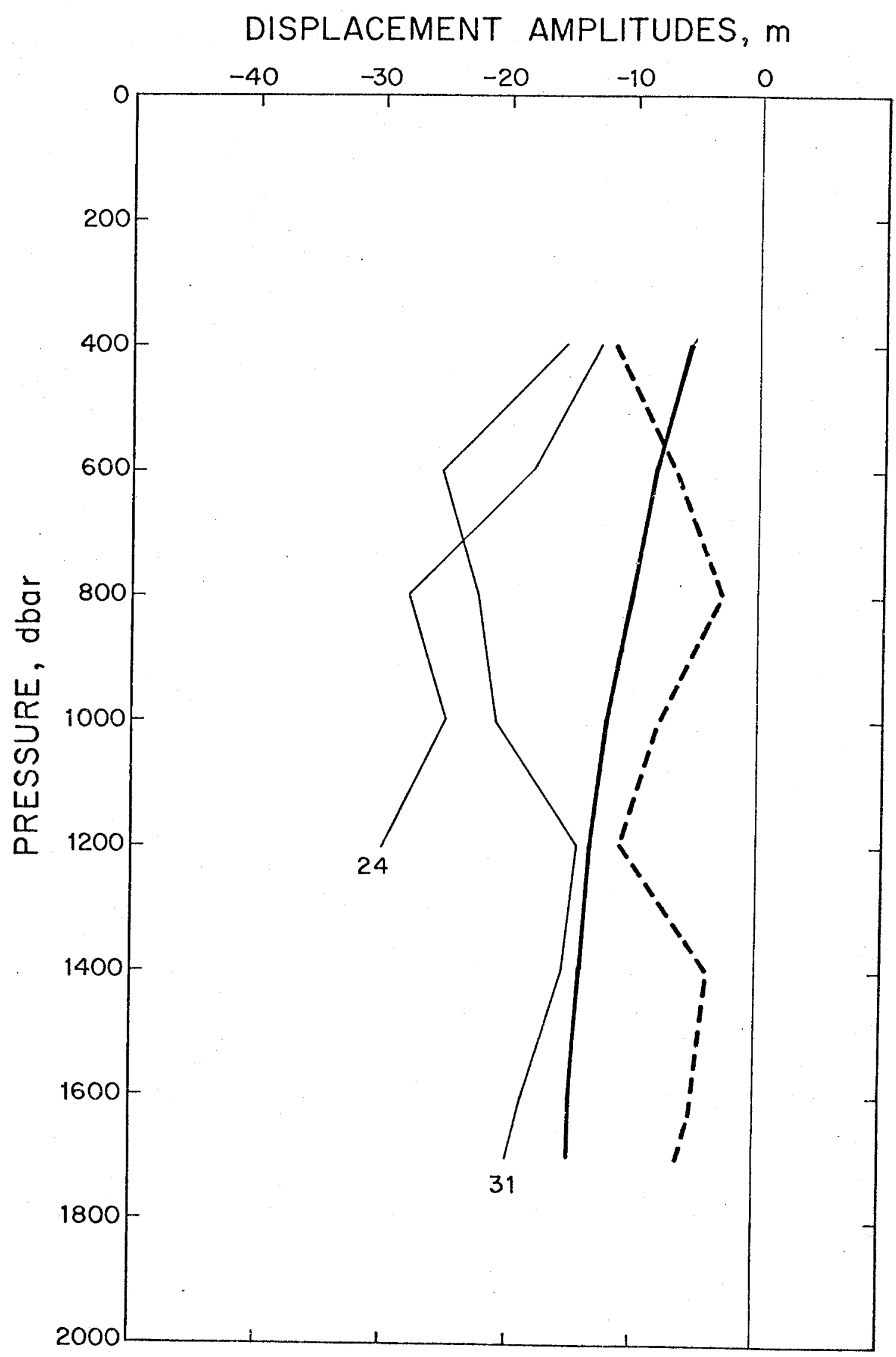


as the annual displacements. a calculated above. (See Figures 3.9 and 2.5.) An estimate of the observed annual component of APE can be made using the displacement amplitudes $a_{\pi}$ :

$$
\mathrm{APE}_{\text {annual }}=\cdot 5 \cdot \mathrm{N}^{2} \cdot \overrightarrow{\mathrm{a}^{2}}
$$

which is in agreement with the model prediction (Fig. 3.9) of the motions predicted by the model, only the forced wave has sufficiently long horizontal scale to explain the changes in the reference steric field, which is independent of horizontal position. The observed seasonal signal in the reference steric field corresponding to a vertical displacement of $O(-15 \mathrm{~m})$ at the thermocline is not well explained by the model, which predicts a displacement of $O(+5 \mathrm{~m})$ at the base of the mixed layer, decreasing monotonically below, for the forced wave. 
Figure 3.9 APE per unit mass: heavy solid line is the model prediction $\left(.5 \mathrm{~N}^{2} \zeta^{2}\right)$ and triangles are $\mathrm{APE}$ annual given by equation (3.27) . 
135

$$
.5 N^{2} \zeta^{2}\left(10^{4} \mathrm{~J} \cdot \mathrm{kg}^{-1}\right)
$$

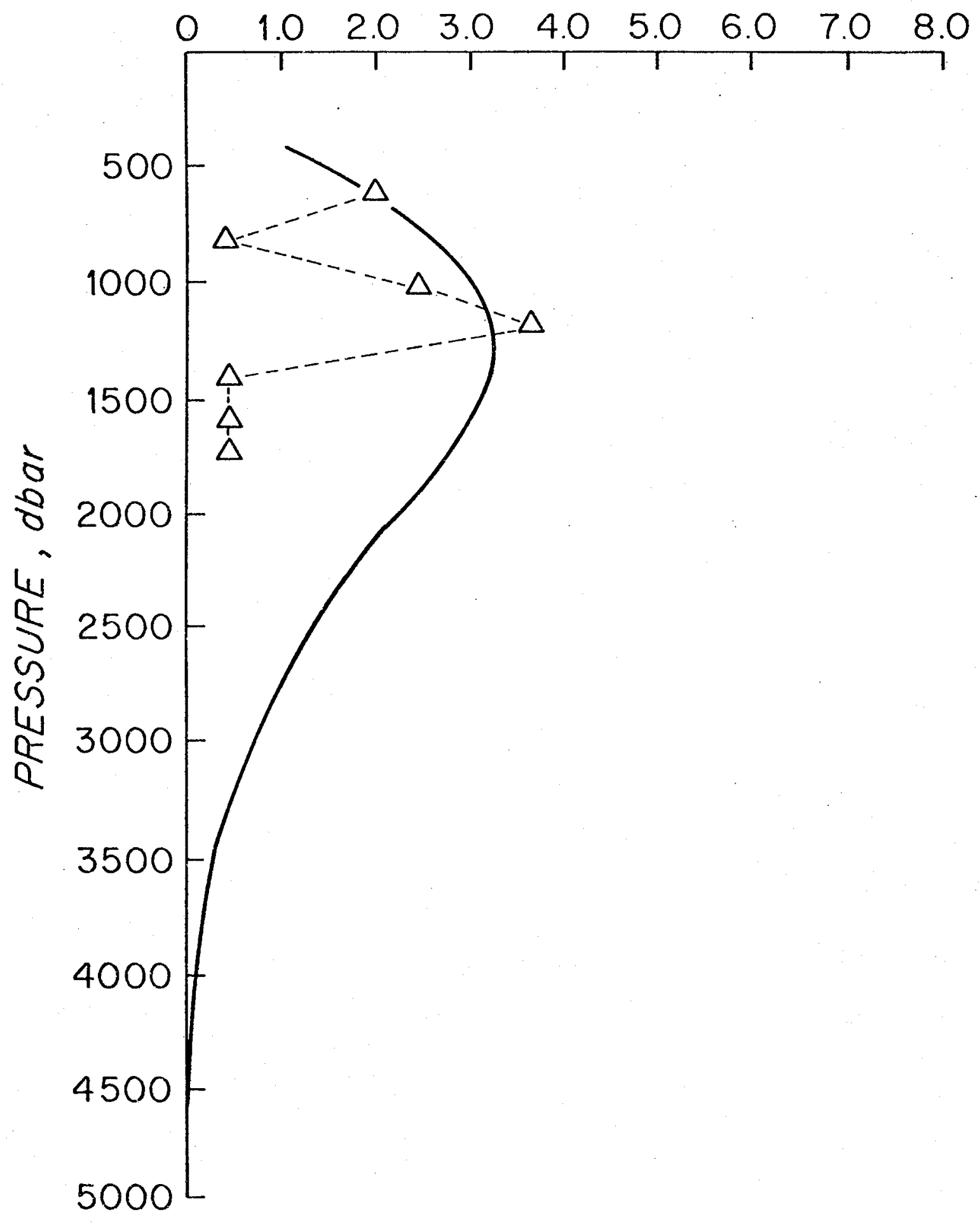




\subsection{Discussion}

In this chapter an analytical model of seasonal atmospheric forcing and oceanic response was constructed in an attempt to answer a number of questions raised by the observational results of Chapter 2. Foremost among those questions was that of the horizontal scale of the seasonal signal in salinity anomaly. Secondly, the question of forcing was posed: what types of atmospheric forcing might result in the observed seasonal signals, with regard to both horizontal scale and amplitude? Finally, what other variables might be expected to show corresponding signals consistent with the model forcing?

In the model ocean, the response consists of two parts: a forced wave with scales comparable to the atmospheric forcing, and a set of free waves required to satisfy the boundary condition at a meridional eastern barrier. The dominant fxee response away from the coast was found to be the first baroclinic mode of wavelength $390 \mathrm{~km}$. The barotropic wave is longer than the basin width, and has very small displacements. The second and higher modes are found to be trapped within $20 \mathrm{~km}$ of the coast. Thus, without any assumptions about the scale or amplitude of the forcing, the scale of the free annual signal in the interior has been established, and it has been determined that no shorter Rossby waves can escape the coastal boundary region.

In order to determine the relative importance of the forced and free waves in the interior further assumptions about the forcing must be made. For simplicity, the model forcing is assumed to be windstress forcing; this is mainly because more data on the distribution of windstress are available than on other types of atmospheric forcing. However, since the windstress forcing is further assumed to act as a body force 
through the mixed layer, so that it affects the remainder of the water column only through an imposed vertical velocity at the base of the mixed layer, the model is certainly compatible with other types of furcing. The horizontal scales of the model forcing, estimated from the observations compiled by Bunker and Worthington (1976), correspond to wavelengths of 12,000 and $6,000 \mathrm{~km}$ in the zonal and meridional directions respectively. The amplitudes used in the model are .1 and -.04 pascals (1. and -0.4 dyne-cm ${ }^{-2}$ ) for $\tau^{x}$ and $\tau^{y}$ respectively. The application of this forcing to the model ocean results in estimates of vertical and horizontal displacements for the free and forced waves. The meridional particle speed of the first baroclinic wave is approximately equal to the phase speed $\left(0\left(.01 \mathrm{~m} \mathrm{sec}^{-1}\right)\right.$; for the barotropic wave the meridional velocity is $0\left(10^{-4} \mathrm{~m} \mathrm{sec}^{-1}\right)$ and for the forced wave $0\left(10^{-3} \mathrm{~m} \mathrm{sec}^{-1}\right)$. The change in the zonal structure of the salinity anomaly field predicted by the model is estimated by the product of the model horizontal displacement and the observed steady meridional gradient in salinity anomaly. For comparison with the model prediction, the observations were fit to a traveling plane wave of wavelength $390 \mathrm{~km}$. The phase and amplitude were both estimated from the observations. The estimated phase in salinity anomaly agrees with the observed phase of windstress within the erxors of phase determination.. The vertical structure of the model and the observed salinity anomaly amplitude agree very well. The amplitude of the signal using all cruises except Phygas 42 is a maximum of -12 ppm, compared to $-16 \mathrm{ppm}$ predicted by the model. The individual cruises 24 , 31. 42, 44 have larger absolute amplitudes, of order 25 to $30 \mathrm{ppm}$. Phygas 42 is the only cruise for which the amplitude is positive; its vertical structure is indistinguishable from the other cruises. A positive 
amplitude is predicted by the model only for positive windstress curl. Although not generally the case in this part of the North Atlantic, positive curl represents something like one to two standard deviations of the observed stresses from the 25 year means, at the model horizontal scales.

The observed vertical displacements were also fit to a traveling plane wave of $390 \mathrm{~km}$ wavelength; the amplitude and vertical structure of the observed signal agrees well with that of the model. This is an independent test of the consistency of the model and of the forcing amplitude. The observed vertical displacement amplitude is $0(15 \mathrm{~m})$ for the $390 \mathrm{~km}$ wave.

The observations of seasonal variability in the zonal structure of salinity anomaly and vertical displacement are therefore consistent with the proposed model of windstress forcing, with respect to amplitude, phase, vertical and horizontal structure. The observed seasonal signal in the reference steric field and the apparently seasonal oscillations in the APE below the main thermocline are not adequately explained by the model; in the former case the observed signal is a factor of 3 too small and has the wrong phase, and in the latter the amplitude of the observed signal is more than an order of magnitude larger than the model prediction.

The model presented in this chapter is simplified in order that it be analytically tractable. Specifically, friction, diffusion, and bottom topography are ignored, the complex geometry of the coastline has not been incorporated, and the region is assumed to be semi-infinite, with no western boundary. The neglect of bottom topography is not serious, as most of the observations are from the area of the Biscay Abyssal Plain. Estjmates of the size of the friction term relative to the time derivative 
term in the momentum equation are found in Table 3.3. Friction is apparently not important for the first baroclinic mode. Lateral diffusion has a stronger effect on the solution: from the estimate given in Table 3.3 one might expect an exror of $25 \%$ in the predicted salinity anomaly signal due to the neglect of diffusion, for a diffusion coefficient of $10^{3} \mathrm{~m}^{2} \mathrm{sec}^{-1}$. For the present case that error is a maximum of $7 \mathrm{ppm}$, less than the measurement exror in salinity.

The coastline geometry used in the model is a simple meridional barrier which is a reasonable approximation of the eastern coast of the North Atlantic over a scale of $5000 \mathrm{~km}$. Locally, however, the coastal geometry is more complicated; in the Bay of Biscay it will be noted that the eastern boundary (taken as the shelf break) lies at an angle of perhaps $45^{\circ}$ west of north. If the model assumed an angled barrier of infinite extent, then there is a critical angle for which all free baroclinic waves are coastally trapped (Philander, 1978). For the parameters used in this model that critical angle is $30^{\circ}$ west of north. However, the constraint of infinite extent is unrealistic, especially since the northsouth extent of the angled coastline is considerably less than the north-south scale of the forcing. The observations suggest that the primary effect of the angled boundary is an alteration of the phase of the free response, since the best fit of the data to $\cos (k x-\omega t-\phi)$ used the distance from the $600 \mathrm{~m}$ isobath as the $\mathrm{x}$-coordinate. It should be noted that in the absence of a model which includes the coastal geometry, this suggestion is not justified theoretically. A proper solution to the theoretical problem might involve a separation of the region into a triangular section bounded by the Iberian Peninsula to the south, the coast of France to the east and noxth and the meridian at $9^{\circ} \mathrm{W}$; and the open 
ocean west of the triangular section. Separate solutions for each area, with matching conditions along $9^{\circ} \mathrm{W}$, could then be found. The solution in the triangular section will most likely involve Bessel functions in the horizontal structure, which introduces a practical difficulty in the comparison of such a model with data.

The model assumes that the region is semi-infinite, implying that the western boundary is sufficiently far way that it has no effect on the solution locally. In the absence of friction both waves will eventually reach a western boundary. A small amount of friction would make the western boundary effectively much farther away for the relatively short baroclinic wave. Even if both waves generated at the eastern boundary actually encounter the western boundary, and reflect, the reflected waves, with eastward group velocity, will have such small scales that oniy a small amount of friction would prevent them from propagating back across the basin to the eastern barrier. Therefore the assurption of a semi-infinite region is acceptable, provided a small amount of friction is included in the argument.

In summary, the observations indicate seasonal signals in salinity anomaly of amplitude $\pm 25 \mathrm{ppm}$ and vertical displacement of amplitude $\pm 15 \mathrm{~m}$, with a zonal wavelength of $390 \mathrm{~km}$ and a phase of 71 days from 1 January, with statistical confidence exceeding $90 \%$. The model presented in this chapter, for which the forcing is an annual signal in windstress with horizontal wavelengths of 12000 and $6000 \mathrm{~km}$ in the zonal and meridional directions, amplitudes of .1 and -.04 pascal and phase corresponding to a maximum at 55 days from 1 January, predicts the observed salinity anomaly and vertical displacement signals with regard to wavelength, vertical structure, amplitude and phase within the errors of the observations. 


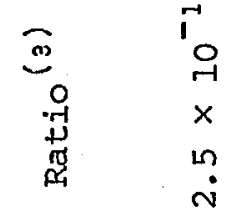
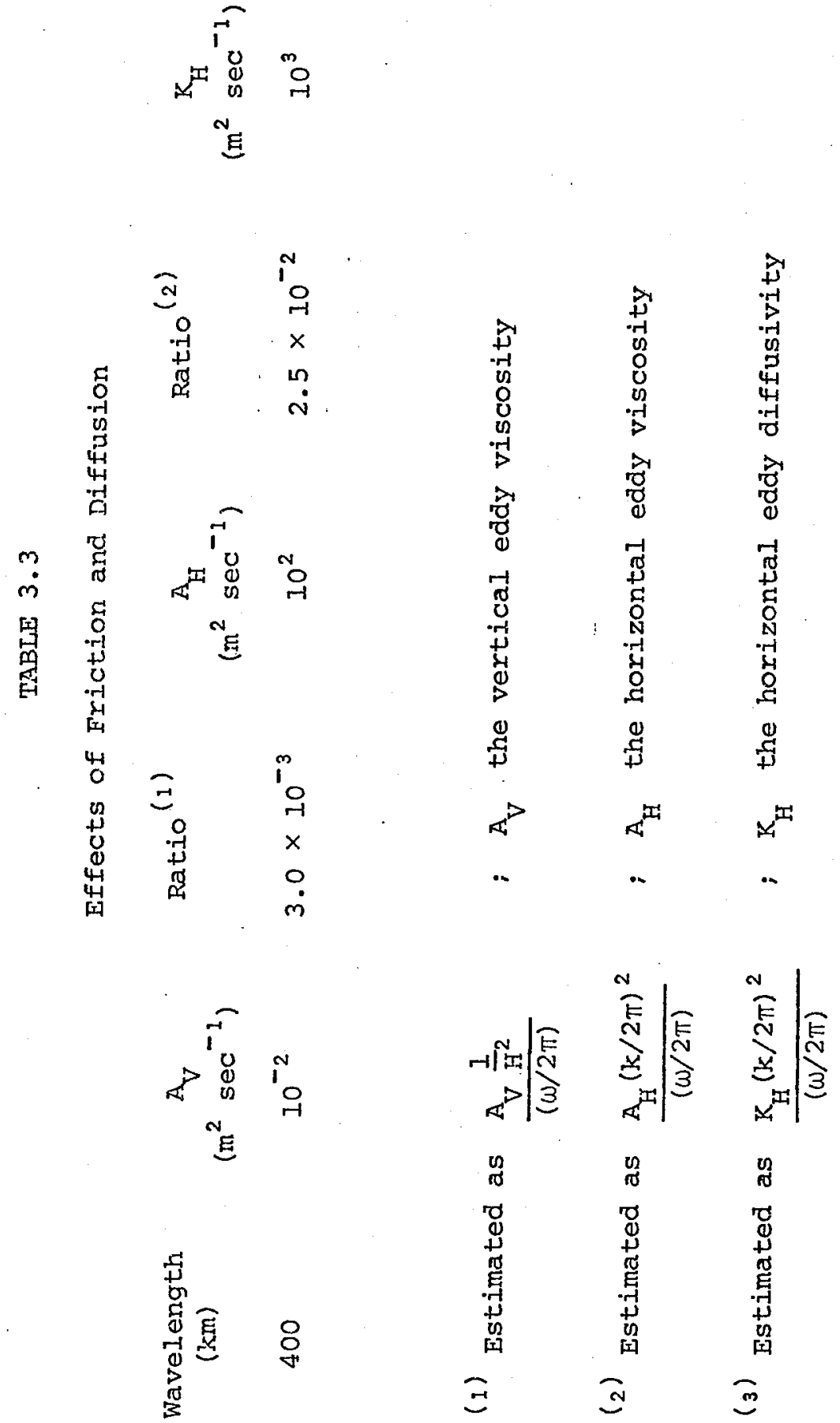


\section{CHAPTER 4}

This work had two primary and three corollary objectives. The primary objectives were: 1) to demonstrate observational evidence for seasonal variability below the main thermocline, and 2) to determine theoretically the types of atmospheric forcing which are consistent with the observations. The corollary objectives were: 1) to identify precisely from observations those material surfaces which are appropriate to the study of tracers in the ocean, 2) to establish a general technique for examining the horizontal distribution of tracers along those surfaces, and 3) to determine from observations the oceanic available potential energy (APE) with particular emphasis on identifying the errors in the estimate of APE. In this chapter the success of those endeavors is analyzed, the implications of the results are discussed, and suggestions are made for possible further work.

\subsection{Results}

The hydrographic observations in the Bay of Biscay have been examined by calculating the adiabatically leveled reference steric field appropriate to each cruise, the distribution of the tracer salinity anomaly along those reference steric surfaces, the vertical displacement of the observed steric surface from the reference surface for each cruise as a function of depth and horizontal position, and the available potential energy (APE). The three-year duration of the data, together with the sampling interval of three months and the large spatial extent of the data in both horizontal dimensions permits the evaluation of seasonal variability in salinity anomaly, vertical displacement, and the reference steric field itself with high statistical confidence. The salinity anomaly field experiences seasonal fluctuations which appeax as westward propagating plane waves with zonal 
wavelength $390( \pm 50) \mathrm{km}$. The disturbance is $180^{\circ}$ out of phase with the seasonal component of the zonal wind stress which has a maximum value at 55 days from 1 January. The computed phase in salinity anomaly corresponds to a minimum at $71( \pm 30)$ days from 1 January. The amplitude of the seasonal signal in salinity anomaly varies with depth, with a maximum amplitude of $30( \pm 10) \mathrm{ppm}$ between 1000 and $1200 \mathrm{db}$ (Fig. 3.6). There is also a seasonal signal in the vertical displacement field, with the same horizontal structure observed in salinity anomaly. The signal in displacement also varies with depth, with structure corresponding to that expected in vertical displacement for a first baxoclinic mode. The maximum amplitude is $30( \pm 7) \mathrm{db}$.

The reference steric field oscillates seasonally with an amplitude of $1 \times 10^{-8}\left( \pm .5 \times 10^{-8}\right) \mathrm{m}^{3} \mathrm{~kg}^{-1}\left(1 \pm .5 \times 10^{-5} \mathrm{~cm}^{3} \mathrm{gm}^{-1}\right)$. Since the reference steric field is independent of horizontal position, it appears that this fluctuation occurs over horizontal scales at least as large as the area 3 covered by the observations. The phase of that oscillation corresponds to a maximum at $130( \pm 30)$ days from 1 January.

The APE per unit mass in this region has two maxima in the vertical: one corresponding to the thermocline, and the second at about $1400 \mathrm{db}$. The thermocline APE is comparable in magnitude to that computed in MODE (Bray and Fofonoff, 1980) with a typical values of $70 \times 10^{-4} \mathrm{~J} \mathrm{~kg}^{-1}\left(70 \mathrm{~cm}^{2} \mathrm{sec}^{-2}\right.$ ) (Fig. 2.6). The second maximum is comparable in magnitude to the thermocline maximum for most cruises and exceeds the thermocline values for some cxuises (Fig. 2.9). There is visual similarity in the vertical structure for cruises which occur in the same seasons; Phygas 31 and 41 which are winter cruises and Phygas 43, a summer cruise have the strongest deep maxima relative to the reference level at $1100 \mathrm{ab}$, with amplitudes of approximately $30 \times 10^{-4} \mathrm{~J} \mathrm{~kg}^{-1}$ relative to the reference level $\mathrm{APE}$. 
Measurements of eddy kinetic energy from moored current meters located. at $47^{\circ} \mathrm{N}, 10^{\circ} \mathrm{W}$ allows comparison of the ratio of APE to kinetic energy pex unit mass as a function of depth. At the thermocline the ratio is approximately 3 to 1 while at 1500 db it is 8 to 1 .

The APE per unit area (or TGPE) between 300 and 1100 db has a mean value over the eleven cruises of $.5 \times 10^{-4} \mathrm{~J} \mathrm{~m}^{-2}$, with a standard deviation of $.2 \times 10^{-4} \mathrm{~J} \mathrm{~m}^{-2}$ (Table 2.5). Bray and Fofonoff (1980) calculated TGPE between 300 and $2500 \mathrm{db}$ for five two-week time windows during MODE; the mean value was $.8 \times 10^{-4} \mathrm{~J} \mathrm{~m}^{-2}$ with standard deviation of $.1 \times 10^{-4} \mathrm{~J} \mathrm{~m}^{-2}$.

The change in potential energy of the system corresponding to changes in the reference steric field from one cruise to the next was also calculated (Table 2.5). That change in potential energy shows no trend over the three year period.

In Chapter 3 it was demonstrated that the observed seasonal signals in salinity anomaly and vertical displacement are consistent with a model which hypothesizes forcing by large scale periodic windstress patterns on a B-plane ocean with a surface mixed layer, depth variable stratification and a meridional eastern barrier. The historical meteorological data for the North Atlantic was used to estimate the forcing parameters, and the stratification and mixed layer parameters were determined from the hydrographic data. The model ocean response to the atmospheric forcing has two parts: a forced wave whose horizontal scales are those of the forcing, and free waves whose meridional scales correspond to that of the forcing and whose zonal scales are determined primarily by the stratification. only the barotropic and first baroclinic modes are not coastally trapped. The long baroclinic mode (wavelength $390 \mathrm{~km}$ ) is found to be the only mode 
which can be important in the interior since the long barotropic mode is very muci longer than the basin, and therefore has negligible velocities and vertical displacements. The model predicts the observed phase and horizontal and vertical structure of both salinity anomaly and vertical displacements within the errors of the observations. The amplitude of the windstress used in the model was chosen such that the model prediction of the salinity anomaly signal agreed reasonably well with the observations. The predicted displacement amplitude agrees with the observations, which is an independent test of the consistency of the model. The model. further predicts changes in horizontal and vertical. velocities, APE and density. The predicted APE per unit mass agrees in amplitude and vertical structure with the annual component of the observed APE per unit mass. That component is calculated as $\cdot 5 \mathrm{~N}^{2} \mathrm{a}_{\pi}^{2}$, with $a_{\pi}$ the amplitude of the seasonal changes in vertical displacement $\pi$. The changes predicted by the model for the large scale density field (corresponding to the forced wave) are a factor of three smaljer than the observed change in the reference steric field, and have different phase anọ vertical structure. The predicted meridional velocities are a maxi... mum of $.018 \mathrm{~m} \mathrm{sec}^{-1}$ at the base of the mixed layex decreasing to $.010 \mathrm{~m}$ $\sec ^{-1}$ at $1000 \mathrm{db}$; the vertical structure is that of the first baroclinic mode. The zonal velccities predicted by the model axe of order $10^{-5} \mathrm{~m} \mathrm{sec}^{-1}$. With regard to the corollary objectives of this work, it was found that the adiabatic leveling technique described by Bray and Fofonoff (1980) permits the precise identification of surfaces appropriate for examining tracer distributions. In this type of analysis, it is important that a minimum of vertical averaging be done in determining potential density surfaces in oraer that resolution is not impaired, but sufficient 
that statistical reliability is achieved. The analysis of observations presented above could not have been accomplished using "surfaces" of constant potential density calculated as the average over some range in potential density: too much information is lost in order to reduce the finestructure erroxs. The adiabatic leveling technique uses a least squares method to weigh the information available from all data points within a vertical interval to determine the reference steric surface. Information about the structure of the density field is thereby retained, allowing better resolution of the reference surface with smaller finestructure errors. In addition, the technique provides an objective estimate of those errors. An important further advantage of the adiabatic leveling technique from the perspective of data analysis is that vertical displacements and APE are also calculated accurately using the refererce steric field; the technique thereby provides a consistent overall framework for the simultaneous analysis of observations in terms of tracers and energetics.

Given the distribution of some tracer along the reference steric surface, it becomes necessary to develop a method for quantifying that distribution---the second corollary objective. The method employed in this work exploits minimization by least squares: the general approach is to choose a possible structure or sum of structures (polynomials or sinusoids) and calculate the amplitude of each structure using a least squares fit of the data. This approach allows the determination of the statistical confidence that the chosen structure in fact exists in the observations, and what the error in the determined amplitude is. The drawback is that the bias of the type of structure expected must be supplied by the investigator. 
The thixd corollary objective was the accurate estimation of APE, particularly the determination of the errors involved in that estimate. It was found that the Boussinesq approximation to APE per unit mass is accurate to about $15 \%$ in most applications; the exror introduced by neglecting the effects of compressibility is not generally significant. However, errors in the numerical integration over pressure in the calculation of APE can be large if the vertical structure is not resolved, so that care must be taken in choosing the vertical separation of points used in the integration. In cases where the vertical displacements of density surfaces are large, the Boussinesq approximation may become in-!, valid; there the gravitational potential energy (GPE) calculation, which is exact, must be used in preference to the Boussinesq which is one term in a Taylor series expansion about displacement. Care must also be exercised in the definition of the reference steric field: if the reference field used does not conserve mass in the transformation to the observed field, large systematic errors in the calculation of GPE may result. Thus, in calculating GPE for an isolated feature such as a Gulf Stream ring, the density field away from the ring cannot be used as the reference field (Reid, Elliott, and Olson, 1980). 


\subsection{Implications}

The steady circulation of the ocean is presumed to be driven primarily by the steady component of the windstress. The seasonal fluctuations in the windstress are of the same order of magnitude as the mean annual values, which suggests that there may be a significant seasonal component to the general circulation. The observational and theoretical results described in the previous section suggest that there are seasonal changes of meridional velocity in the eastern North Atlantic of order $\pm .01 \mathrm{~m} \mathrm{sec}^{-1}$ with zonal wavelength of about $400 \mathrm{~km}$ and meridional wavelength of perhaps $6000 \mathrm{~km}$. The short zonal scale suggests that horizontal averaging could replace temporal averaging in distinguishing this annual signal from the long-term mean flow. Recall from Chapter 1 that the mean interior flow in the outer Bay of Biscay region has been estimated as less than $.01 \mathrm{~m} \mathrm{sec}^{-1}$ by Swallow, et al. (1977).

The relationsip of horizontal scale to amplitude of measurable quantities such as displacements and velocities is central to this observational work and its interpretation. A finite total energy is transferred from the atmosphere to the ocean during the seasonal cycle. That energy is transferred over large horizontal scales, so that the associated displacements are relatively small. If, by some unspecified mechanism, all or part of that energy is converted to smaller horizontal scales, the relative magnitude of the displacements will increase. The central hypothesis of this work is that just such a mechanism is found in the reflection of a large scale, atmospherically forced wave at a meridional barrier. The shortness of horizontal scale which permits the observation of these seasonal signals limits their direct effect upon large scale oceanic phenomena such as the general circulation and the zonally averaged 
meridional heat flux. However, the smallex scale annual signals can influence the interpretation of measurements of large scale quantities unless adequate spatial or temporal averages are used. For that reason alone it is important to know the expected scales and corresponding ampIitudes of the annual signal in the ocean.

The study of large scale circulation through examination of tracer distributions has a long history in oceanography: initially, because direct current meter measurements were not available, and more recently because the large scale tracer distributions represent a time integraI of the velocity field, with the small scale variability in velocity smoothed. With the observations now available in which tracer distributions car be studied as a function of time, the low frequency variability of the general circulation can be inferred by techniques like those used in this work. The method used here is appropriate to regions in which there are steady horizontal gradients of tracers; there are a wide range of conditions under which the method may be usefully applied. Figure 4.1 is an illustration of possible solutions of the one dimensional equation for the conservation of salinity anomaly:

$$
s_{t}+v(x) s_{y}=0
$$

with different values of $v$ plotted as functions of $s_{t}$ and $s_{y}$ (Values of $s_{t}$ and $s_{y}$ which are less than the probable errors at frequency $I$ cpy and scale $500 \mathrm{~km}$ are hatchea.) For example, in order to detect by this indirect a method a velocity signal of amplitude $1 \mathrm{~cm} \mathrm{sec}^{-1}$ and frequency $1 \mathrm{cpy}$, a steady gradient of at least . $2 \mathrm{ppm} \mathrm{km}^{-1}$ is necessary.

Finally, the calculation of APE presented in this work has some implications for the study of ocean energetics. Previous work using hydrographic data from the MODE experiment (Kim 1975; Bray and Fofonoff, 1980) 
Figure 4.1 Particle velocities as a function of lateral salinity anomaly gradient and change of salinity anomaly with time from equation (4.1). Hatched areas represent values less than the probable errors for frequency $I$ cpy and horizontal scale $500 \mathrm{~km}$. 


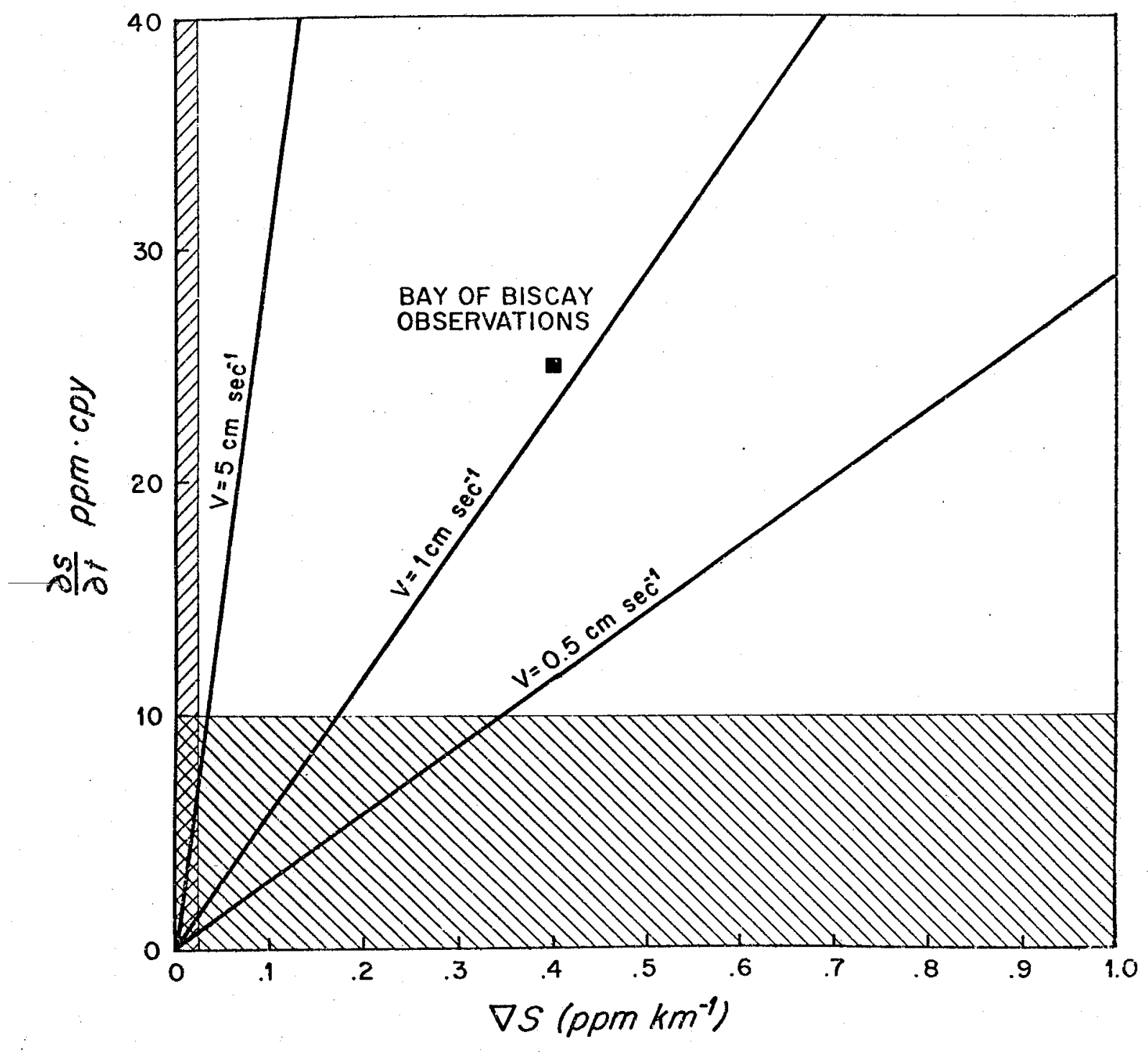


indicated a simple vertical structure for APE per unit mass, resembling the average buoyancy frequency profile, which has a maximum at the thermocline, and decreases monotonically below. The amplitude of the MODE APE, which was calculated over a region $400 \times 400 \mathrm{~km}$ in size, nowhere in the water column exceeded the observed low frequency kinetic energy (KE) by more than a factor of 3 . In the Bay of Biscay observations, APE pex unit mass has a second maximum below the thermocline of approximately equal amplitude; the amplitude and the deep vertical structure both change significantly with time. Furthermore, the ratio of APE to $\mathrm{KE}$ below the thermocline in the Bay of Biscay observations (Fig. 2.6) is larger than for MODE, with a value at $1200 \mathrm{db}$ of $8: 1$. The difference may be due in part to the larger area over which APE is calculated in the Bay of Biscay. Nevertheless, it appears that there is an excess of APE over KE in this part of the eastern North Atlantic, as well as significant low fxequency variations in the vertical structure and amplitude of APE below the thermocline. 


\subsection{Further Work}

The observations presently available limit further progress on the problem of the oceanic response to seasonal atmospheric forcing: refinement of the theoretical work presented in this thesis is limited in its usefulness by the sophistication and completeness of the observations to which the theory is applied. An array of hydrographic stations with more dense spatial coverage might allow comparison with more complicated models (involving, for example, the coastline geometry in more detail); a shorter sampling period would permit the study of higher frequency phenomena; a longer time series would give not only more statistical confidence to the result, but would provide evidence of interannual variability of the signal. The meteorological observations presently available should be examined for details of the structure of the low frequency wind field, with particular attention to the direction of propagation. An update of the meteorological observations between 1972 and the present would be a valuable addendum to the work of Bunker and Worthington (1976), especially for synoptic comparison of the wind field and the large scale oceanographic experiments which have been carried out in that interval.

The general technique for data analysis presented here might be applied with success to the equatorial oceans, where the expected signal is strong; as an example, in the western equatorial Indian Ocean there are strong meridional gradients of salinity anomaly resulting from the intrusion of high salinity water from the Arabian Sea. Hydrographic and velocity measurements of considerable extent and duration are available for that area (Luyten and Swallow, 1976), and the theory of low frequency equatorial waves is well developed (Philander, 1978). Eriksen (1980) 
discusses observational evidence of equatorially trapped waves in the western part of the Indian Ocean, using moored current meter data. The adiabatic leveling technique would be improved by extension to depths shallower than $300 \mathrm{~m}$, where it is presently invalid. Such an extension requires that a method be developed for handling density surfaces which are not continuous over the region considered. A similar problem may be encountered at depth in regions of abrupt, isolated topographic features. 


\section{ACKNOWLEDGMENTS}

I am indebted to Nick Fofonoff for his expert and patient guidance during the course of my thesis work. Discussions with Glenn Flierl added significantly to the work presented in Chapter 3. Harry Bryden's thorough criticism improved the manuscript. Bill Schmitz was largely responsible for motivating me during the final stages of this work.

I would also like to acknowledge with gratitude the influence of my parents, whose example set me this challenge, and that of Gil corcos, whose interest and enthusiasm steered me toward graduate study in physical oceanography. Joy Geiselman's friendship helped me to maintain a stable perspective on graduate work. Dean Roemmich shared with me all the day by day trials and successes which eventually compose a work such as this; without his companionship it would not have been completed.

The manuscript was typed by Audrey Williams, whose patience did not falter through several revisions. The figures were drafted principally by Dorothy Meinert. This work was supported by the Office of Naval Research under contract N00014-76-C-197, NR 083-400. 
Abramowitz,M. and I.A. Stegun (1972) Handbook of Mathematical Functions, Dover, 1046p.

Bray, N. A. and N. P. Fofonoff (1980) Available Potential Energy for MODE Eddies, submitted to J. Phys. Oceanography.

Bryan, K. and P.Ripa (1978) The vertical structure of North Pacific Temperature Anomalies, Journal of Geophysical Research 83, 2419-2429.

Bunker, A.F. and I.V. Worthington (1976) Energy exchange charts of the North At lantic Ocean, Bulletin of the American Meteorological Society $57(6), 670-678$.

Eriksen, C.C. (1980) Evidence for a continuous spectrum of Equatorial Waves in the Indian Ocean, Journal of Geophysical Research $85(\mathrm{c} 6), 3285,3303$.

Firing, E. (1978) Seasonal Oscillations in a Mid-Latitude Ocean with Barriers to Deep Flow, Ph.D. Thesis, Joint Program in Oceanography M.I.T. -Woods Hole, 265 pp.

Fofonoff, N.P. (1962) Physical properties of sea water. In: The Sea: Ideas and Observations, Interscience Publishers, London, 3-50.

Fofonoff, N.P. (1977) Computation of potential temperature of seawater for an arbitrary reference pressure. Deep-Sea Research 24, 489-491.

Fofonoff, N. P. and H. L. Bryden (1975) Specific gravity and density of seawater at atmospheric pressure. J. Mar. Res., Supplement, vol. $33,69-82$.

Fofonoff, N. P., S. P. Hayes and R. M. Millard (1974) WHOI/Brown CTJ Microprofiler: Methods of calibration and data handling, Woods Hole Oceanographic Institution Technical Report WHOI 74-89, 64pp

Fruchaud, B. (1975) Etude Hydrologique et variations saisonnieres dans le proche Atlantique en 1972, Rapp. Scient. Techn. CNEXO No. 20, $44 \mathrm{pp}$.

Fruchaud-Laparra, B., J. Le Floch, J. Y. Le Tareau, A Tanguy (1976a) Etude Hydrologique et variations saisonnieres dans le proche At 1antique en 1973, Rapp. Scient. Techn. CNEXO No. 26, 111p. 
Fruchaud-Laparra, B., J. Le Floch, C. Le Roy, J.L. Le Tareau, F. Madelain (1976b) Etude Hydrologique et variations saisonnieres dans le proche Atlantique en 1974, Rapp. Scient. Techn. CNEXO No. 30, 108pp.

Gascard, J. (1980) Mid Oceanic Eddy in the northeastern Atlantic, Polymode News 76, 9-10, unpublished manuscript.

Gregg, M.C. (1979) The effects of bias error and system noise on parameters computed from $C, T, P$ and $V$ profiles. Journal of Physical Oceanography $9(1), 200-217$

Iselin, C. O'D (1939) The influence of vertical and lateral turbulence on the characteristics of waters at mid-depths. Transactions, American Geophysical Union, 414-417.

Katz, E.J. (1970) Diffusion of the core of Mediterranean Water above the mid-Atlantic Ridge crest, Deep-Sea Research, 17, 611-625

Kim, K. (1975) Instability and energetics in a baroclinic ocean, Ph.D. Thesis M.I.T. and W.H.O.I. Joint Program $175 \mathrm{pp}$.

Lorenz, E.N. (1955) Available Potential Energy and the Maintenance of the General Circulation, Tellus, ㄱ, 157-167.

Luyten, J.R. and J.C. Swallow (1976) Equatorial Undercurrents, Deep-Sea Research 23(10), 999-1001.

Montgomery, R.B. (1938) Circulation in upper layers of southern North At lantic deduced with use of isentropic analysis, Papers in Physical Oceanography and Meteorology, 6 (2), 1-48.

Needler, G.T. and R.A. Heath (1975) Diffusion coefficients calculated from the Mediterranean salinity anomaly in the North At lantic Ocean Journal of Physical Oceanography, ㄴ, 173-182.

Niiler, P.P. and W.S. Richardson (1973) Seasonal Variability of the Florida Current, Journal of Marine Research 31, 144-167.

Pedlosky: J. (1979) Geophysical Fluid Dynamics, Springer-Verlag, New York, $624 \mathrm{pp}$.

Philander, S.G.H. (1978) Forced Oceanis Waves Reviews of Geophysics and Space Physics i6(1), 14-46.

Reid, J.L. and R.J. Lynn (1971) On the influence of the Norwegian-Greenland and Weddell seas upon the bottom waters of the Indian and Pacific oceans, Deep-Sea Research 18, 1063-1088. 
Reid, R.O., B.A. Elliott and D.B. Olson (1980) Available potential energy: a clarification, submitted to Journal of Physical Oceanography.

Swa11ow, J.C. (1969) A deep eddy off Cape St. Vincent, Deep-Sea Research 16(Supplement), 285-295.

Swallow, J.C., W.J. Gould and P.M. Saunders (1977) Evidence for a poleward eastern boundary current in the North Altantic Ocean, International Counci for the Exploration of the Sea, Contribution to Statutory Meeting CM 1977/C:32 (unpublished manuscript).

Veronis, G. and H. Stomel (1956) The action of variable windstress on a stratified ocean Journal of Marine Research 15, 43-75.

White, W.B. (1977) Annual forcing of baroclinic long waves in the tropical North Pacific Ocean Journal of Physical Oceanography $7(1), 50-61$.

White, W.B. (1978) A wind-driven model experiment of the seasonal cycle of the main thermocline in the interior mid-latitude North

Pacific Journal of Physical Oceanography $8(5), 818-824$

Worthington, L. V. and W. G. Metcaif (1961) The relationship between potential temperature and salinity in deep Atlantic water. Rapp. et P. V. Conseil Perm. Exp. de 1a Mer, Vol. 149, 122-128.

Wunsch, C. (1972) Bermuda seal level in relation to tides, weather and baroclinic fluctuations, Reviews of Geophysics and Space Physics
$10(1), 1-49$. 
Appenaix A: Calculation of Systematic and Random Error in $\mathrm{APE}$

1. Systematic differences between $G P E$ and $A P E_{B}$

The inclusion of compressibility effects in GPE was shown in Chapter 1 to introduce systematic differences between GPE and $A P E_{B}$. Those differences were interpreted as contributions from horizontal and vertical gradients of compressibility, and it was noted that $-\alpha_{\mathrm{p}}^{*} \bar{j}$ can be replaced by $\overline{k \pi}$ in the horizontal term. It should also be noted that the computational algorithm calculates $\alpha_{p}^{*}\left(p_{i}\right)$ which differs from $\alpha_{p}^{*}\left(p_{f}\right)$. Differentiating (1.14) with $\psi=\alpha$ :

$$
\frac{d \alpha_{i}}{d p}\left(p_{i}\right)=\frac{d \alpha_{f}}{d p}\left(p_{f}\right)\left[1+\frac{d \pi}{d p}\right]+k\left(p_{i}\right)-k\left(p_{f}\right)\left[1+\frac{d \pi}{d p}\right]
$$

or

$$
\alpha_{p}^{*}\left(p_{i}\right)=\alpha_{p}^{*}\left(p_{f}\right)\left(1+\frac{d \pi}{d p}\right)
$$

Thus $\overline{\alpha_{p}^{\star} \pi}$ may be rewritten as

$$
\alpha_{p}^{*}\left(p_{f}\right) \pi=\alpha_{p}^{*}\left(p_{i}\right) \pi+\frac{d}{d p}\left(\frac{\alpha \pi^{*}}{2}\right) \frac{\pi^{2}}{2} \frac{d \alpha_{p}^{*}}{d p}
$$

This expansion may also be used to evaluate the contribution from horizontal gradients of compressibility; however, errors in the determination of the second derivative of $\alpha$ make it less satisfactory than the simpler $\overline{\mathrm{KT}}$. The corresponding errors in TGPE are given by 


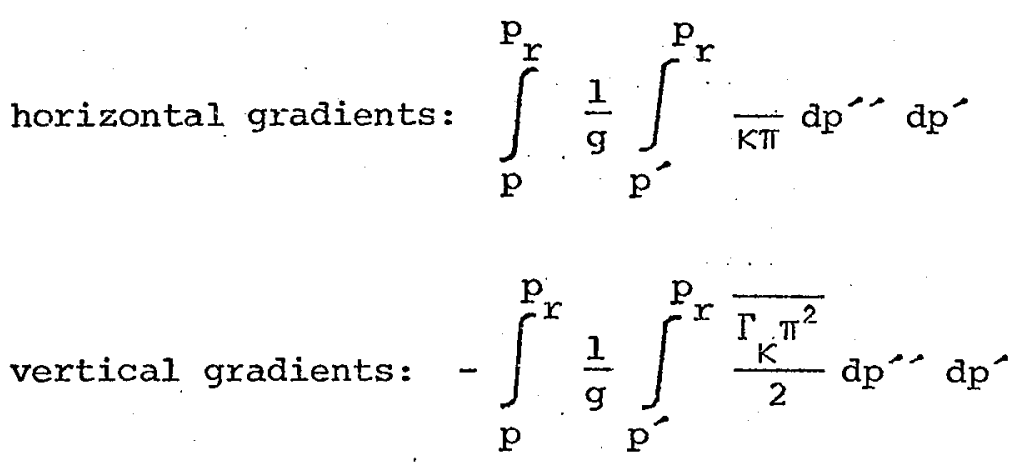

The contributions of these terms to TGPE between 300 and $1100 \mathrm{db}$ are given in Table 2.5; the contributions to GPE for Phygas 42 are plotted in Fig. 2.6.

2. Random errors

A general expression for the variance of HPE (1.11) may be derived by assuming that all errors are uncorrelated from station to station and from level to level in the vertical, and that the variance of $\frac{\alpha *}{p}$ is small compared to that of the displacement $\pi_{k}$. Then, in terins of suns over the integration steps $\Delta p$, the variance of HPE is:

$$
\begin{aligned}
V(H P E) & =v\left[\sum_{j=j(p)}^{j\left(p_{r}\right)}\left[\frac{I}{M} \sum_{k=1}^{M} \frac{1}{2 g} \alpha_{p}^{*} \cdot \pi_{k}^{2}\right]_{j} \Delta p_{j}\right] \\
& =\sum_{j=j(p)}^{j\left(p_{r}\right)}\left[\frac{1}{M} \sum_{k=1}^{M} \frac{\alpha^{*^{2}}}{4 g} v\left(\pi_{k}^{2}\right)\right] \Delta p_{j}^{2} .
\end{aligned}
$$

Here $M$ is the number of stations and the subscript $j$ refers to the level. The variance of $\pi_{k}^{2}$ is given by

$$
V\left(\pi_{k}^{2}\right)=2\left[V\left(\pi_{k}\right)\right]^{2}+4<\pi_{k}>^{2} v\left(\pi_{k}\right)
$$


with $<\pi_{k}>$. the ensemble mean of $\pi_{k}$, which is estimated by $\pi_{k}$ itself. The finestructure variance of $\pi_{k}$ is taken to be the variance of the regression estimate $p$, which is described in Chapter 2 . The finestructure errors in HPE are found in Table 2.5 .

Expressions for the variance of TGPE and GPE are most readily derived using the expansion of $\left(\alpha_{i}-\alpha_{f}\right)$ from equation (1.14). (This is because $\alpha_{i}$ and $\alpha_{f}$ are cbtained by inverting high order polynomials.) If any covariance between the terms in the perturbation expansion of TGPE are ignored, the variance of TGPE is then estimated as:

$$
\begin{aligned}
V(T G P E) & =v\left[\sum_{j=j(p)}^{j\left(p_{x}\right)} \frac{l}{g}\left[\sum_{\ell=\ell(p)}^{\ell\left(p_{x}\right)}\left[\frac{1}{M} \sum_{k=1}^{M}\left(\alpha_{p}^{*}+\frac{\Gamma_{k} \pi^{2}}{2}\right)\right] \Delta p_{\ell}\right] \Delta p_{j}\right] \\
& +v\left[\sum _ { j = j ( p ) } ^ { j ( p _ { x } ) } \frac { 1 } { 2 g } \left[\frac{1}{M} \sum_{k=1}^{M} \underset{p}{\left.\left.\alpha * \pi^{2}\right] \Delta p_{j}\right]}\right.\right.
\end{aligned}
$$

$$
=\sum_{j=j(p)}^{j\left(p_{r}\right)}\left[\frac{1}{M} \sum_{k=1}^{M} \frac{\alpha *^{2}}{4 g} v\left(\pi_{k}^{2}\right)+\sum_{\ell=\ell(p)}^{\ell\left(p_{r}\right)} \frac{1}{g^{2}}\left[\frac{1}{M} \sum_{k=1}^{M} \frac{\Gamma^{2}}{4} v\left(\pi_{k}^{2}\right)+v\left(\frac{1}{M} \sum_{k=1}^{M} \alpha_{p}^{*} \pi\right) \Delta p_{\ell}^{2}\right] \Delta p_{j}^{2}\right.
$$

with $v\left(\pi_{k}^{2}\right)$. given by (A 3). The third term in (A 4) requires some care in evaluation, because $\bar{\pi}$ is constrained to be zero. From section 1 of the Appendix:

$$
\overline{-\alpha \pi}=\bar{\kappa} \pi
$$

$\alpha$ is very nearly constant; if it is taken to be a constant, the variance of $\overline{k \pi}$ becomes

$$
v(\overline{\kappa \pi})=v(K \cdot \bar{\pi})
$$




$$
=k^{2} \vee(\bar{\pi})
$$

In practice, $\bar{\pi}$. j.s not found to be exactly zero, due to numerical errors, so that some small contribution is to be expected from the third term. The variance of $\bar{\pi}$ is estimated as $(\pi)^{2}$. Values of the finestructure errors for TGPE are found in Table 2.5 and for GPE in Fig. 2.6. Because the finestructure errors in the compressibility terms are small, the error in $\mathrm{APE}_{\mathrm{B}}$ differs very little from that in GPE.

Measurement errors for GPE and TGPE were calculated by assuming that only the pressure measurement error contributes to errors in $\pi_{k}$ (i.e., that temperature and salinity errors do not contribute) and that measurement exrors in $\alpha_{p}^{*}$, and $\Gamma_{k}$ may be ignored. A value for the pressure error of $\pm 5 \mathrm{db}$ was used as the standard deviation of $\pi_{k}$ in (A 5). Contributions to the total measurement error from the exror in the compressibility terms were found to be negligible; hence the measurement error in $A P E_{B}$ is effectively the same as that calculated for GPE using (A 4). The measurement error is plotted for GPE in Fig. 2.6 and tabulated for TGPE in Table 2.5. 
Appendix B: Cubic Spline Coefficients for Iselin - Worthington - Metcalf $\theta$-S Curve

(L. Armi and C. Maillard, personal communication)

\begin{tabular}{|c|c|c|c|c|}
\hline $\mathrm{T}_{0}$ & $\mathrm{C}_{0}$ & $\mathrm{C}_{1}$ & $\mathrm{C}_{2}$ & $c_{3}$ \\
\hline$\left({ }^{\circ} \mathrm{C}\right)$ & (ppt) & & & \\
\hline 0 & 34.738063 & 0 & 0 & 0 \\
\hline .5 & 34.738053 & .107290 & $.584849 \mathrm{E}-2$ & $-.253429 \mathrm{E}-2$ \\
\hline 1.20 & 34.815152 & .111753 & $.523726 E-3$ & $.582151 E-1$ \\
\hline 1.50 & 34.850297 & .127785 & $.529320 \mathrm{E}-1$ & -.135379 \\
\hline 1.75 & 34.883436 & . 128868 & $-.485828 \mathrm{E}-1$ & -.129913 \\
\hline 2.0 & 34.910587 & $.802174 \mathrm{E}-1$ & -.146093 & .228920 \\
\hline 2.25 & 34.925087 & $.500936 \mathrm{E}-1$ & $.255484 \mathrm{E}-1$ & $-.267382 \mathrm{E}-1$ \\
\hline 2.50 & 34.938790 & $.578544 \mathrm{E}-1$ & $.552526 \mathrm{E}-2$ & $-.359945 \mathrm{E}--1$ \\
\hline 2.75 & 34.953036 & $.538681 \mathrm{E}-1$ & $-.214953 \mathrm{E}-1$ & $-.374594 \mathrm{E}-1$ \\
\hline 3.00 & 34.964575 & $.360969 \mathrm{E}-1$ & $-.495364 \mathrm{E}-1$ & $.509274 \mathrm{E}-1$ \\
\hline 3.20 & 34.970220 & $.223936 \mathrm{E}-1$ & $-.189292 \mathrm{E}-1$ & $.580683 E-1$ \\
\hline 3.40 & 34.974406 & $.217901 \mathrm{E}-1$ & $.157868 \mathrm{E}-I$ & $.479730 \mathrm{E}-2$ \\
\hline 3.60 & 34.979434 & $.286805 \mathrm{E}-1$ & $.185975 \mathrm{E}-1$ & $-.294172 \mathrm{E}-1$ \\
\hline 3.80 & 34.985679 & $.325895 \mathrm{E}-1$ & $.102958 \mathrm{E}-2$ & $-.279688 E-\mathrm{Y}$ \\
\hline 4.00 & 34.992014 & $.296450 \mathrm{E}-1$ & $-.157123 \mathrm{E}-1$ & $.643397 \mathrm{E}-2$ \\
\hline 5.00 & 35.01 .238 & $.175223 \mathrm{E}-\mathrm{i}$ & $.357759 \mathrm{E}-2$ & $.114377 \mathrm{E}-2$ \\
\hline 7.00 & 35.07089 & $.455579 \mathrm{E}-1$ & $.104386 \mathrm{E}-1$ & $.865592 \mathrm{E}-5$ \\
\hline 10.00 & 35.30174 & .108423 & $.105172 \mathrm{E}-1$ & $-.763343 \mathrm{E}-3$ \\
\hline 13.00 & 35.70106 & .150916 & $.364790 \mathrm{E}-2$ & $.31080 .5 \mathrm{E}-4$ \\
\hline 16.00 & 36.18748 & .173643 & $.392926 \mathrm{E}-2$ & $-.689782 \mathrm{E}-2$ \\
\hline 19.00 & 36.55753 & $.109775 \mathrm{E}-1$ & $-.581443 \mathrm{E}-1$ & $.696380 \mathrm{E}-1$ \\
\hline 21.00 & 36.9040118 & 0 & 0 & 0 \\
\hline
\end{tabular}


Appendix B (cont.)

Salinity is then calculated as

$$
\begin{aligned}
\mathrm{S}_{\theta} & =\mathrm{C}_{0}+\mathrm{C}_{1} \Delta \mathrm{T}+\mathrm{C}_{2} \Delta \mathrm{T}^{2}+\mathrm{C}_{3} \Delta \mathrm{T}^{3} \\
\text { with } \Delta \mathrm{T} & =\mathrm{T}_{\text {obs }}-\mathrm{T}_{0}
\end{aligned}
$$




\section{Biographical sketch}

Nan Bray was born May 5, 3952 in Idaho Falls, Idaho: She attended Stanford University from 1969 to 1971 and the University of California, Berkeley from 1972 until 1975, where she received the B,S. degree in Engineering Physics. She began work in the Joint Program in September of 1975. 